\title{
Computer Modeling and Experimental Verification of Figure-Eight-Shaped Null-Flux Coil Suspension System
}

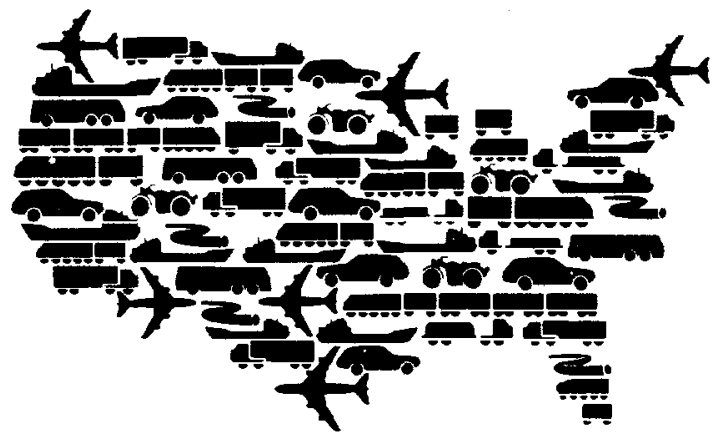

Center for Transportation Research Argonne National Laboratory

Operated by The University of Chicago, under Contract W-31-109-Eng-38, for the United States Department of Energy 


\section{Argonne National Laboratory}

Argonne National Laboratory, with facilities in the states of Illinois and Idaho, is owned by the United States Government, and operated by the University of Chicago under the provisions of a contract with the Department of Energy.

This technical memo is a product of Argonne's Energy Systems (ES)

Division. For information on the division's scientific and engineering activities, contact:

Director, Energy Systems Division

Argonne National Laboratory

Argonne, Illinois 60439-4815

Telephone (708) 252-3724

Presented in this technical memo are preliminary results of ongoing work or work that is more limited in scope and depth than that described in formal reports issued by the ES Division.

Publishing support services were provided by Argonne's Information and Publishing Division.

\section{Disclaimer}

This report was prepared as an account of work sponsored by an agency of the United States Government. Neither the United States Government nor any agency thereof, nor any of their employees, makes any warranty, express or implied, or assumes any legal liability or responsibility for the accuracy, completeness, or usefulness of any information, apparatus, product, or process disclosed, or represents that its use would not infringe privately owned rights. Reference herein to any specific commercial product, process, or service by trade name, trademark, manufacturer, or otherwise, does not necessarily constitute or imply its endorsement, recommendation, or favoring by the United States Government or any agency thereof. The views and opinions of authors expressed herein do not necessarily state or reflect those of the United States Government or any agency thereof.

Reproduced directly from the best available copy.

Available to DOE and DOE contractors from the Office of Scientific and Technical Information, P.O. Box 62, Oak Ridge, TN 37831; prices available from (615) 576-8401. 


\section{DISCLAIMER}

Portions of this document may be illegible in electronic image products. Images are produced from the best available original document. 


\section{Computer Modeling and Experimental Verification of Figure-Eight-Shaped Null-Flux Coil Suspension System}

by J.L. He, T.M. Mulcahey, D.M. Rote, and T. Kelly

Center for Transportation Research, Energy Systems Division, Argonne National Laboratory, 9700 South Cass Avenue, Argonne, Illinois 60439

December 1994

Work sponsored by United States Army Corps of Engineers and the Federal Railroad Administration through interagency agreements E8691R001 and DTFR 53-91-X-00018, respectively, with the United States Department of Energy and by Argonne National Laboratory

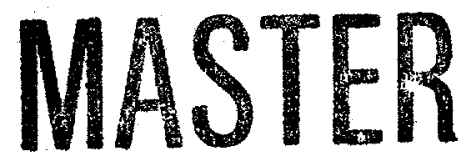




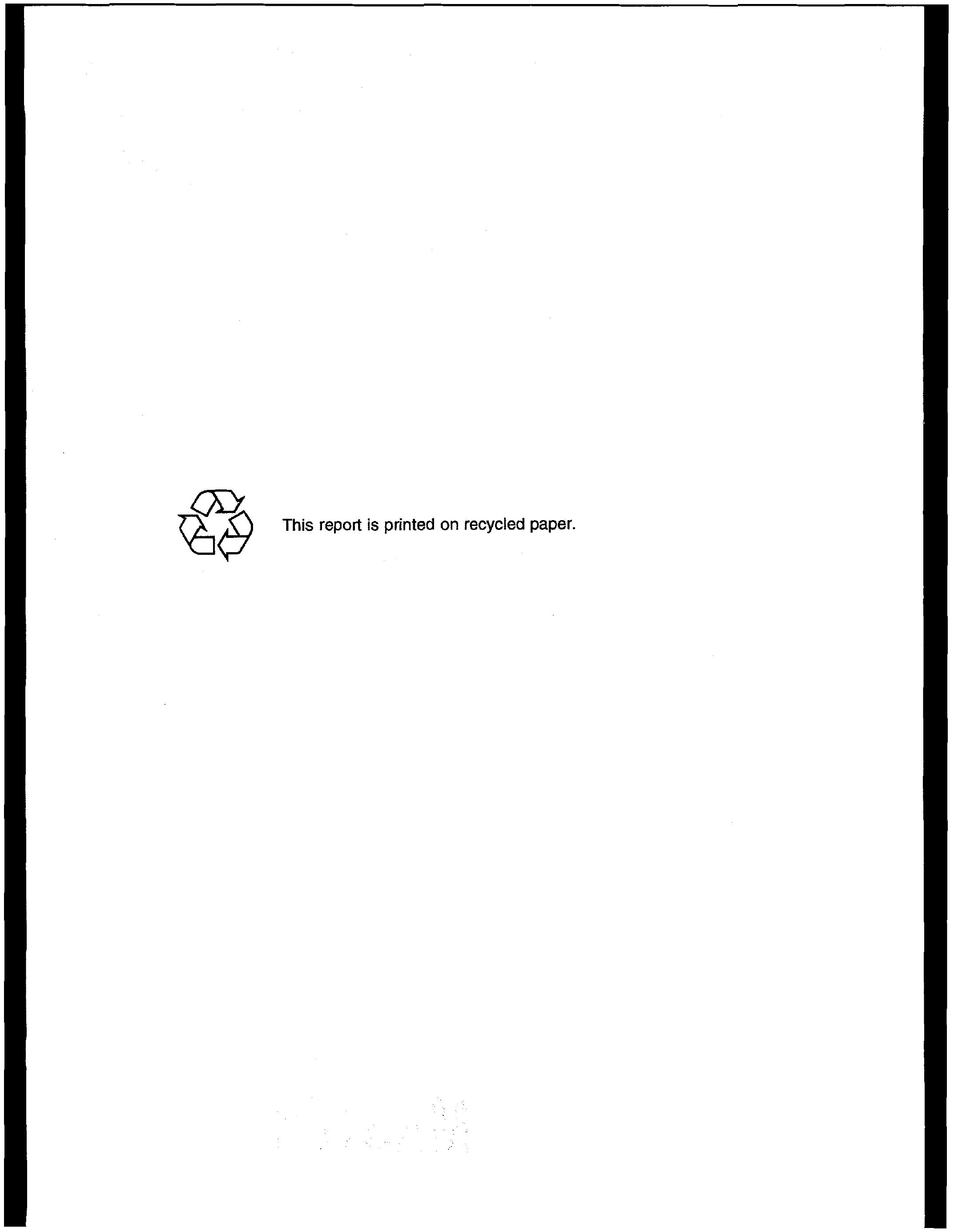




\section{Contents}

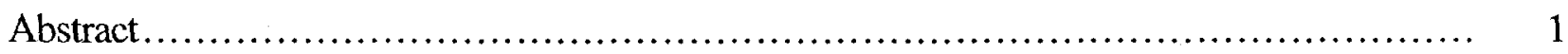

1 Review of Coil-Type Maglev Guideways ................................... 1

2 The Computer Simulation Model ............................................. 6

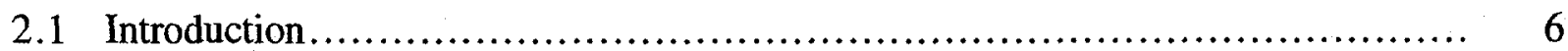

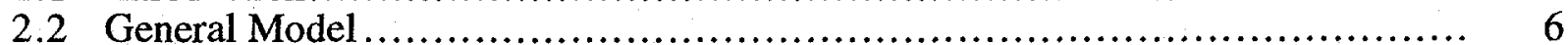

2.3 Determination of Circuit Parameters ............................................. 9

2.3.1 Resistance and Inductance ............................................. 9

2.3.2 Determination of Mutual Inductance................................... 10

2.4 Introduction to the Computer Code ......................................... 13

2.5 Numerical Example ...................................................... 13

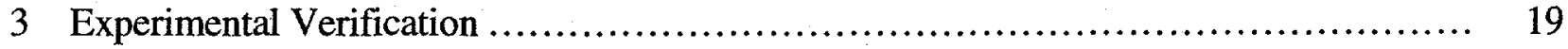

3.1 Experimental Apparatus .............................................. 19

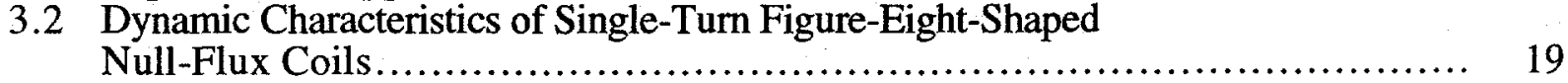

3.3 Dynamic Force Characteristics.............................................. 26

3.4 Dynamic Characteristics of Thick Single-Turn Figure-Eight-

3.5 Multi-Turn Figure-Eight-Shaped Null-Flux Coil Experiment .................... 36

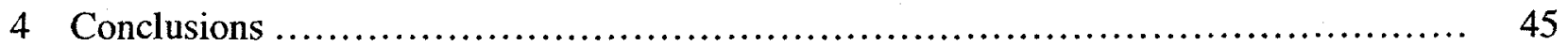

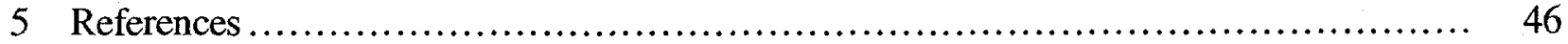

\section{Table}

2.1 Data Used for Computer Simulation ..................................... 16

Figures

1.1 Single-Loop Coil Guideway for Vertical Levitation ............................ 2

1.2 Vertically Arranged Single-Loop Coil Guideway for Null-Flux Lateral Guidance.

1.3 Double-Row-Loop Coil Guideway for Vertical Levitation and

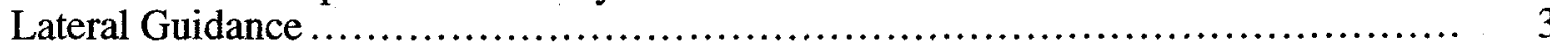

1.4 Figure-Eight-Shaped Null-Flux Coil Guideway for Lateral Guidance ............. 3

1.5 Figure-Eight-Shaped Null-Flux Coil Guideway for Vertical Levitation............. 4 
Figures (Cont.)

1.6 Cross-Connected Figure-Eight-Shaped Null-Flux Coil Guideway

for Vertical Levitation and Lateral Guidance.

1.7 Flux-Cancellation Concept for Vertical Levitation

2.1 Interaction between Two Coils Simplified as the Interaction between Four Effective Conductors.

2.2 Determination of Mutual Inductance between Two Finite-Length Conductors .......

2.3 Determination of Mutual Inductance between Two Rectangular Coils.

2.4 Block Diagram of the Main Program

2.5 Side-Wall Suspension System

2.6 Null-Flux Lift and Drag Force as Functions of Vehicle Velocity

2.7 Null-Flux Lift and Lift-to-Drag Ratio per SCM as Functions of Vertical Displacement.

2.8 Null-Flux Lift as a Function of Time

3.1 Photograph of the Experimental Null-Flux Apparatus ............................ 20

3.2 Schematic of the Experimental Null-Flux Apparatus ............................. 21

3.3 Experimental Null-Flux Apparatus................................................ 22

3.4 Magnet Orientation............................................................. 22

3.5 Null-Flux Coil Configuration Positions in Drum .............................. 23

3.6 Single-Turn Figure-Eight-Shaped Null-Flux Coil Configurations .................. 23

3.7 Measured Magnetic Forces as a Function of Time for Null-Flux

Coil Configurations A through $\mathrm{E}$

3.8 Computer-Calculated Magnetic Forces Acting on Various

Figure-Eight-Shaped Null-Flux Coils, Using the Same

Parameters as in Figure 3.7

3.9 Computer-Calculated Magnetic Forces as a Function of Time for

Null-Flux Coil Configurations A through $E$ at a Speed of $100 \mathrm{~m} / \mathrm{s}$

3.10 Time-Averaged Magnetic Forces of Null-Flux Coil Configuration A as a Function of Speed

3.11 Time-Averaged Magnetic Forces of Coil Configuration A as a Function of Horizontal Displacement at a Speed of $22.6 \mathrm{~m} / \mathrm{s}$ 


\section{Figures (Cont.)}

3.12 Time-Averaged Magnetic Forces of Coil Configuration A

as a Function of Horizontal Displacement at a Speed of $100 \mathrm{~m} / \mathrm{s}$

3.13 Force Characteristics of a Permanent Magnet Approaching a Stationary Guideway Coil.

3.14 Force Characteristics of a Permanent Magnet Departing from a

Stationary Guideway Coil

3.15 Force Characteristics of a Permanent Magnet Passing over a Stationary

Guideway Coil at High Speed or High Time Constant

3.16 Thick Single-Turn Figure-Eight-Shaped Null-Flux Coil

3.17 Magnetic Force Waveforms at a Speed of $22.6 \mathrm{~m} / \mathrm{s}$ and a

Lateral Displacement of $11 \mathrm{~mm}$

3.18 Magnetic Force Waveforms at a Speed of $100 \mathrm{~m} / \mathrm{s}$ and a

Lateral Displacement of $11 \mathrm{~mm}$.

3.19 Dependence of Time-Averaged Magnetic Force on Speed at a

Lateral Displacement of $5 \mathrm{~mm}$....

3.20 Time-Averaged Magnetic Forces as a Function of

Lateral Displacement at a Speed of $22.6 \mathrm{~m} / \mathrm{s}$.

3.21 Time-Averaged Magnetic Forces as a Function of

Lateral Displacement at a Speed of $100 \mathrm{~m} / \mathrm{s}$.

3.22 Calculated Flux Density Produced by a Superconducting Coil

as a Function of Lateral Distance

3.23 Flux Density Measured on Permanent Magnet using

Standard Hall Probe

3.24a Computer-Calculated Lift Force Impulse as a Function

of Time for Nine-Turn Series Coil....

3.24b Experimental Lift Force Impulse as a Function of Time

for Nine-Turn Series Coil.

3.25a Computer-Calculated Guidance Force Impulse as a Function

of Time for Nine-Turn Series Coil

3.25b Experimental Guidance Force Impulse as a Function of Time

for Nine-Turn Series Coil

3.26a Computer-Calculated Drag Force Impulse as a Function

of Time for Nine-Turn Series Coil 


\section{Figures (Cont.)}

3.26b Experimental Drag Force Impulse as a Function of Time for Nine-Turn Series Coil.

3.27a Computer-Calculated Lift Force Impulse as a Function of Time for Two Five-Turn Parallel Coils

3.27b Experimental Lift Force Impulse as a Function of Time for

Two Five-Turn Parallel Coils.

3.28a Computer-Calculated Guidance Force Impulse as a Function of Time for Two Five-Turn Parallel Coils

3.28b Experimental Guidance Force Impulse as a Function of Time for Two Five-Turn Parallel Coils

3.29a Computer-Calculated Drag Force Impulse as a Function of Time for Two Five-Turn Parallel Coils

3.29b Experimental Drag Force Impulse as a Function of Time for Two Five-Turn Parallel Coils. 


\title{
Computer Modeling and Experimental Verification \\ of Figure-Eight-Shaped Null-Flux Coil Suspension System
}

\author{
by
}

\author{
J.L. He, T.M. Mulcahey, D.M. Rote, and T. Kelly
}

\begin{abstract}
This report discusses the computer modeling and experimental verification of the magnetic forces associated with a figure-eight-shaped null-flux coil suspension system. A set of computer codes called COILGDWY, were developed on the basis of the dynamic circuit model and verified by means of a laboratory model. The experimental verification was conducted with a rotating PVC drum, the surface of which held various types of figure-eight-shaped null-flux coils that interacted with a stationary permanent magnet. The transient and dynamic magnetic forces between the stationary magnet and the rotating conducting coils were measured and compared with results obtained from the computer model. Good agreement between the experimental results and computer simulations was obtained. The computer model can also be used to calculate magnetic forces in a large-scale magnetic-levitation system.
\end{abstract}

\section{Review of Coil-Type Maglev Guideways}

The electrodynamic suspension (EDS) magnetic-levitation (maglev) system utilizes the repulsive magnetic force generated from the interaction between vehicle-borne magnets, usually superconducting magnets (SCMs), and the eddy currents induced in the guideway conductors. Various types of guideway conductors have been developed for EDS maglev systems. Two typical guideway conductors are the sheet-type guideway conductors and the discrete-coil-type guideway conductors. The sheet-type guideway conductors were used in many early maglev concepts. Currently, the Magneplane, one of the four U.S. maglev system designs, employs an arc-shaped sheet conductor for levitation and partial guidance. This report focuses on the discretecoil-type guideways, but the model also can be applied to other coil-type guideways.

The horizontal single-loop coil guideway, shown in Figure 1.1, is the simplest coil-type guideway configuration and was used in many early EDS maglev concepts, including early versions of the Japanese MLU system. The single-loop coil-type guideway is frequently considered to be superior to the sheet-type guideway because of its relatively low magnetic drag force. The single-loop coil guideway, however, produces undesirable force pulsations that do not occur in the sheet-type guideway conductors; the amplitude of these force pulsations depends upon 


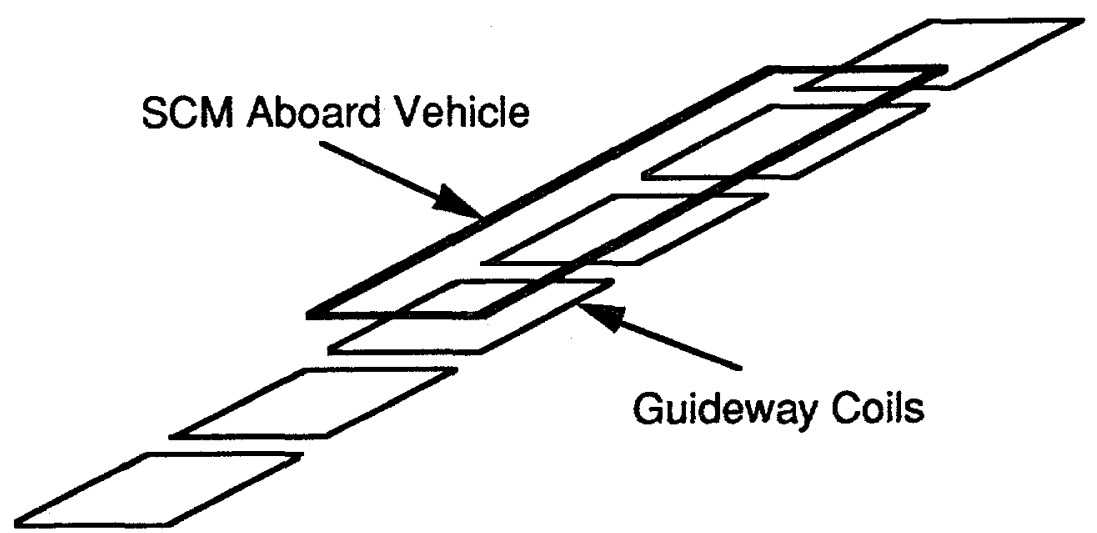

FIGURE 1.1 Single-Loop Coil Guideway for Vertical Levitation

the coil pitch of the guideway. An alternative single-loop coil guideway concept is shown in Figure 1.2, where the horizontally arranged superconducting magnet array aboard the vehicle interacts with the vertically arranged single-loop coil array to produce a lateral guidance force. This configuration, originated in 1969,1 follows the null-flux principle.

The double-row-loop coil guideway shown in Figure 1.3 consists of two arrays of horizontal-loop coils arranged in parallel, interacting with a vehicle-borne magnet array to produce levitation and guidance forces. ${ }^{2}$ Two vehicle-borne magnet arrays aboard the vehicle interact with the two double-row loop coils to levitate and guide a maglev vehicle. In this case, the side-wall of the guideway can be omitted, and the cost of the guideway may be reduced. In addition, the double-row-loop coil guideway can be used to develop a simple electromagnetic guideway directional switch.

The null-flux coil suspension concept invented in 1969 has become very popular in the maglev community. 1 Japanese maglev researchers have further developed several versions of the null-flux coil suspension system, including the side-wall null-flux suspension system. The major advantage of the null-flux coil suspension system is that it can provide very high lift-to-drag ratios, typically several hundred, depending upon design and vertical offset. The horizontally arranged, figure-eight-shaped null-flux coil array (Figure 1.4) was employed in the Canadian maglev concept design for lateral guidance. The vertically arranged, figure-eight-shaped null-flux coil array has been used for side-wall vertical levitation systems, such as the Japanese MLU system (Figure 1.5) and, in the United States, Foster Miller's maglev concept. Recently, the Japanese maglev group has further improved the side-wall levitation system by cross-connecting the nullflux coil arrays mounted on the two side walls as shown in Figure 1.6. The cross-connected figure-eight-shaped null-flux coil suspension system can provide both null-flux levitation and guidance forces, with very high lift-to-drag and guidance-to-drag ratios. 3,4

Figure 1.7 shows a guideway configuration, developed by the Bechtel maglev team, called "flux-cancellation suspension." In this system, the octupole superconducting magnet arrays 


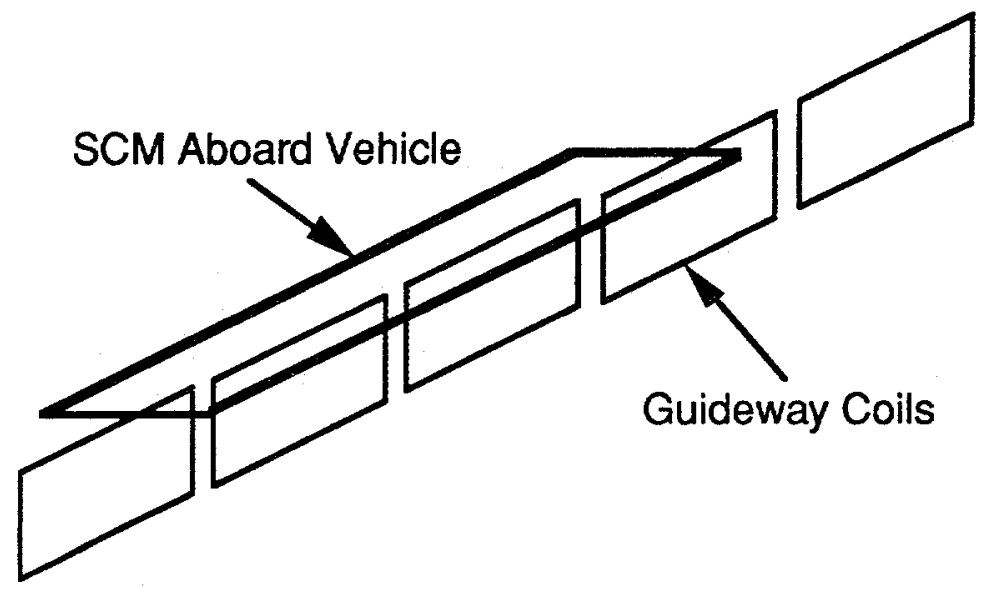

FIGURE 1.2 Vertically Arranged Single-Loop Coil Guideway for Null-Flux Lateral Guidance

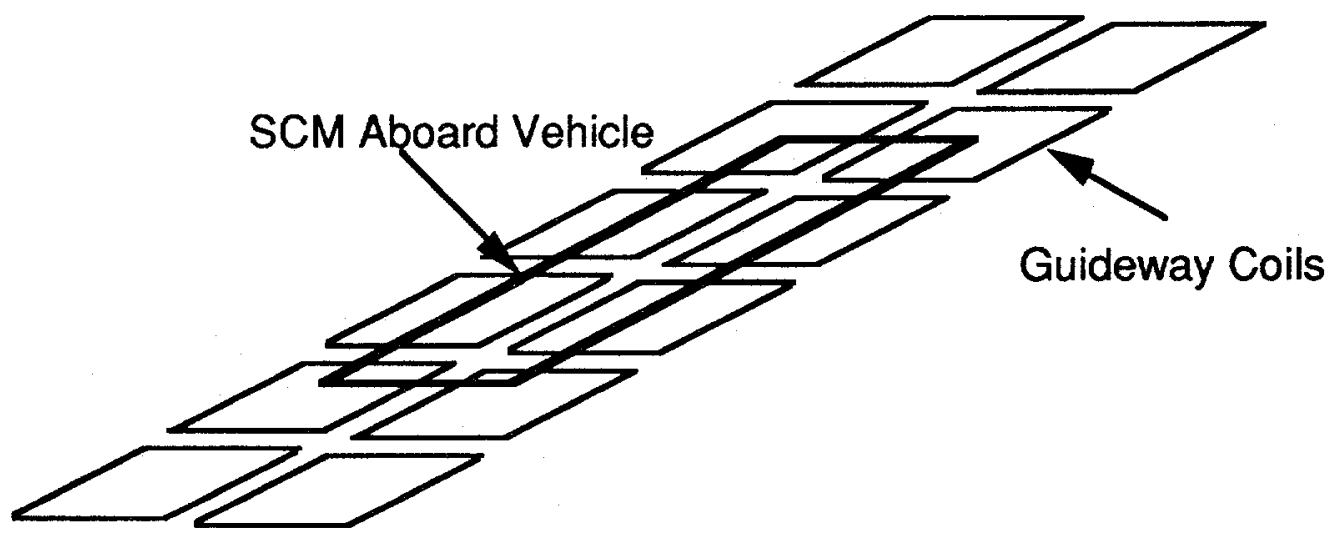

FIGURE 1.3 Double-Row-Loop Coil Guideway for Vertical Levitation and Lateral Guidance

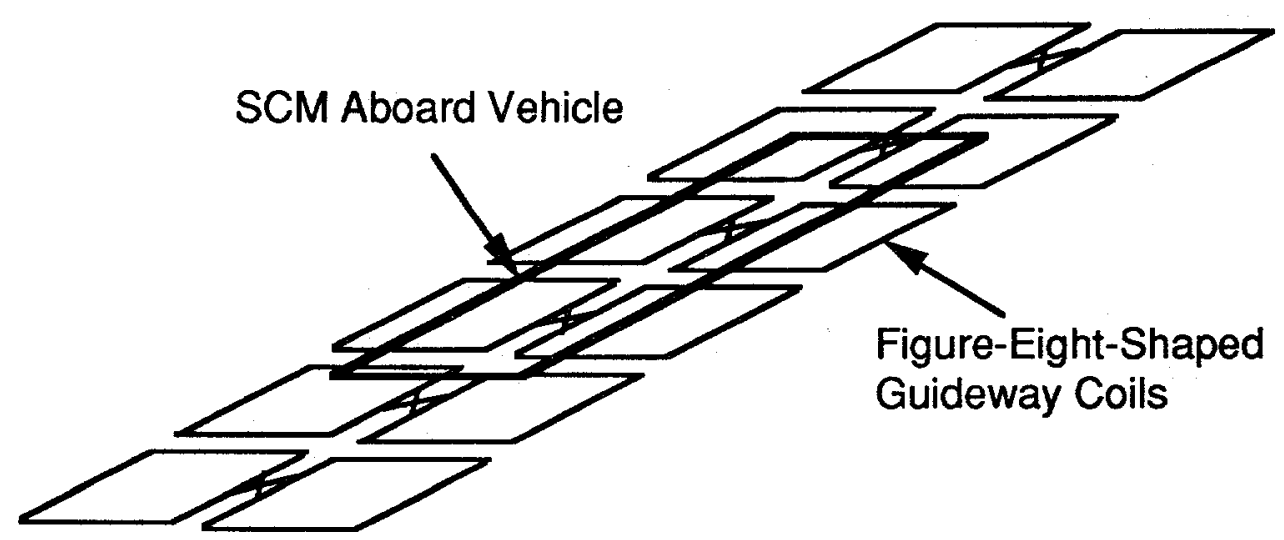

FIGURE 1.4 Figure-Eight-Shaped Null-Flux Coil Guideway for Lateral Guidance 


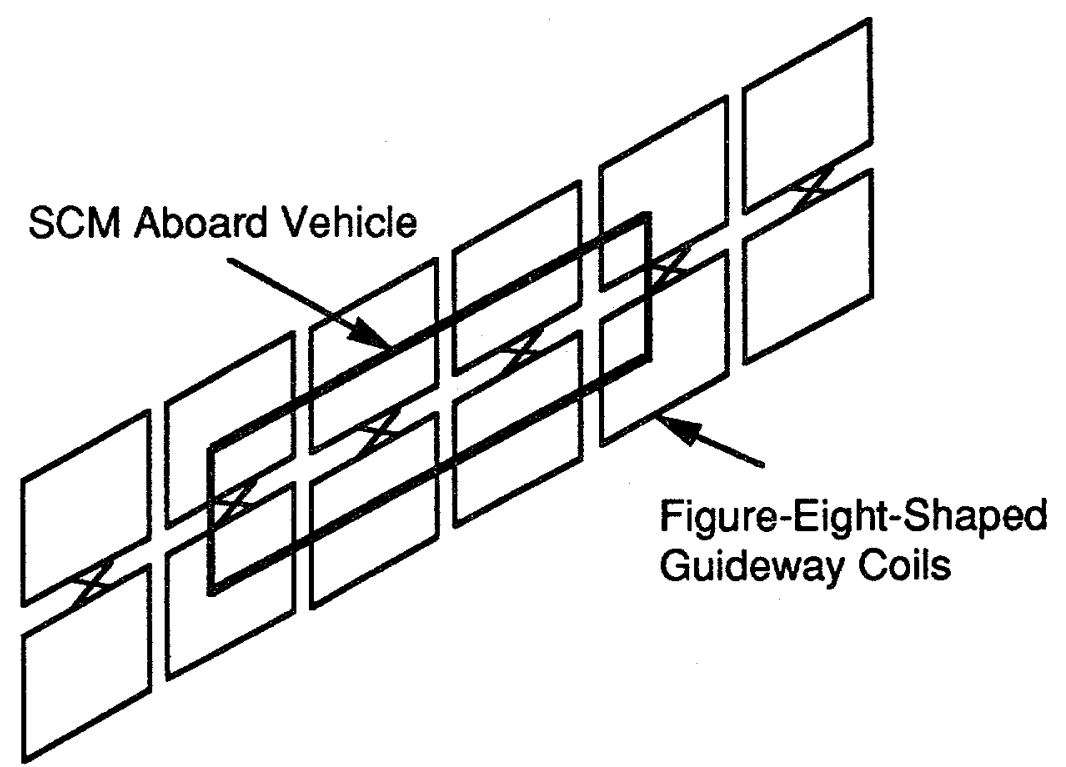

FIGURE 1.5 Figure-Eight-Shaped Null-Flux Coil Guideway for Vertical Levitation

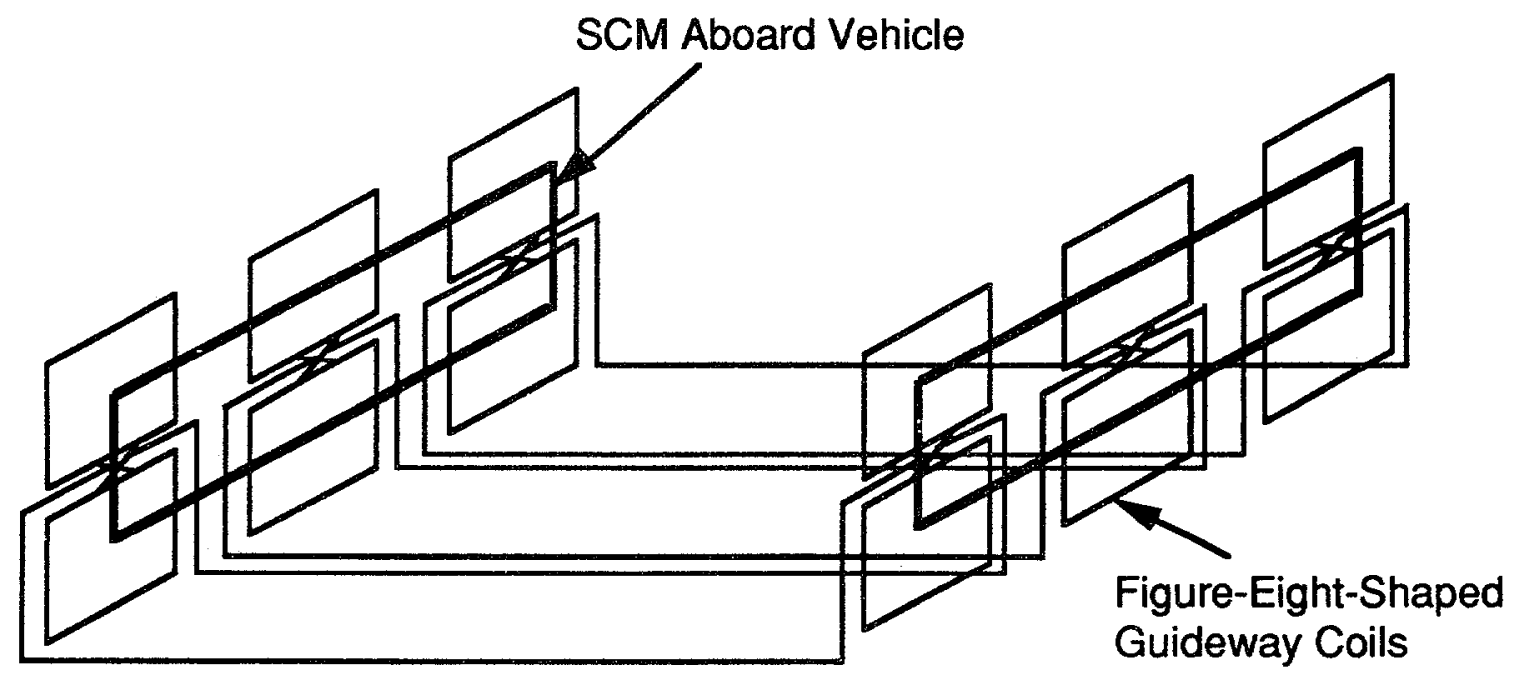

FIGURE 1.6 Cross-Connected Figure-Eight-Shaped Null-Flux Coil Guideway for Vertical Levitation and Lateral Guidance 


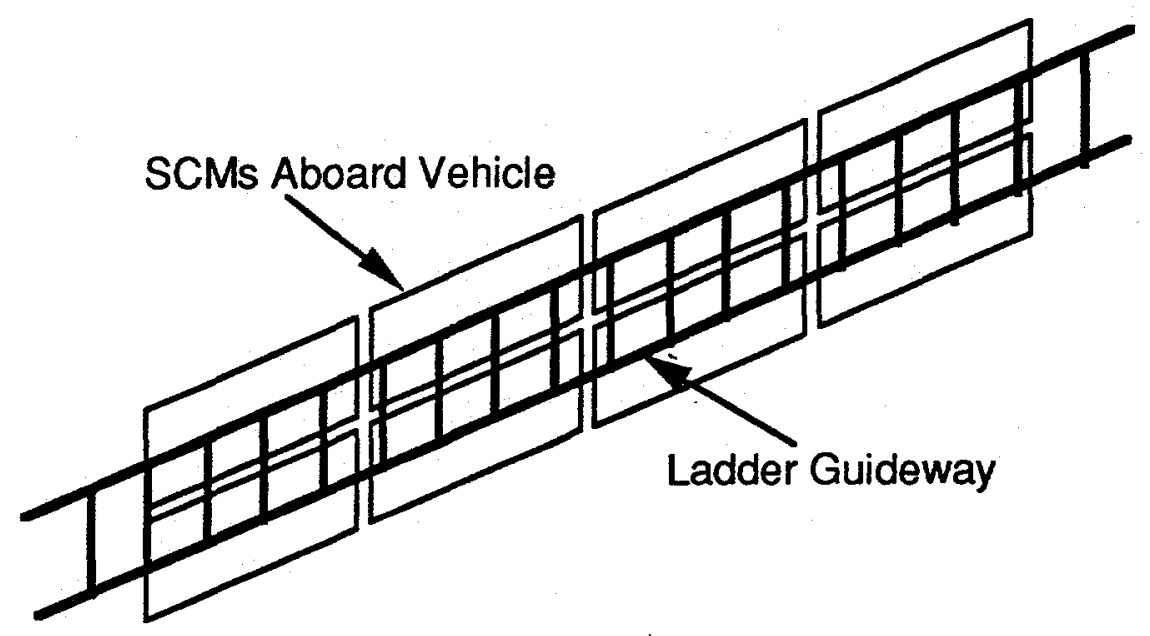

FIGURE 1.7 Flux-Cancellation Concept for Vertical Levitation

aboard the vehicle interact with the vertically arranged ladder guideway conductors to produce the levitation force. The system, in principle, is very similar to the null-flux concept because the net flux passing through the window of the ladder vanishes at the vertical equilibrium position. The approach used for analyzing the ladder guideway is similar to that used in other coil-type guideways previously discussed.

EDS propulsive systems consist of discrete propulsion coils interacting with vehicle superconducting magnets. The propulsive system interacts in a manner similar to the suspension systems. Thus, the model used for the computation of suspension forces can also be applied to the forces associated with propulsive systems.

Investigation of various types of coil-type guideway conductors, as well as propulsion windings, leads to the conclusion that coil-type guideway conductors have several unique features, depending on their configurations. First, various circuit models can be applied to the coil-type guideways. Second, all of the coil conductors used in maglev systems have a rectangular shape which can be treated as four finite-length conductors. The circuit parameters of the coil-type guideway can be obtained easily. Third, no ferromagnetic materials are used in EDS maglev systems, so a linear circuit model will be sufficient for the EDS coil guideway.

It should be noted that the circuit model used for maglev is motion-dependent, and the circuit parameters are functions of time and space as the vehicle moves forward. The dynamic circuit theory is very suited to coil-type maglev system analysis. 


\section{The Computer Simulation Model}

\subsection{Introduction}

The COILGDWY computer program contains several independent computer codes for different coil-type guideways, including the figure-eight-shaped null-flux coil guideway and the loop coil guideway. These codes were developed on the basis of dynamic circuit theory, as discussed in previous papers. ${ }^{5}$ The unique feature of the theory is that it treats the maglev suspension system in terms of space- and time-dependent circuit parameters governed by a set of differential equations in matrix form, taking both end-effects and edge-effects of the system into consideration. Once the system of equations is solved for the current distribution, the forces acting between the vehicle's superconducting magnets and the guideway coils can be calculated in a straightforward manner. Therefore, the performance of the system can be investigated. The equations are usually solved for the currents in the time domain, so the method is well-suited for transient and dynamic analysis and for computer simulation of coil-type maglev systems. In this report, we apply dynamic circuit theory to the figure-eight-shaped null-flux coil guideway and demonstrate the utility and versatility of the approach and the utility of the COILGDWY computer codes. Validation of the COILGDWY computer codes may lead to a better understanding of the dynamic characteristics of the null-flux coil suspension concept.

\subsection{General Model}

A maglev system can be represented by the dynamic circuit model, in which the system energy, power, and forces, as well as other quantities, are expressed in terms of their equivalent circuit parameters. Consider a maglev system in which $\mathrm{m}$ vehicle coils or conductors interact with $\mathrm{n}$ guideway coils or conductors to produce levitation, guidance, and propulsion forces. Assume [i] and [e] to be column $(m+n)$ matrices made up of the individual currents and voltages associated with the vehicle and guideway coils or conductors, respectively; [L] to be a square $(m+n) \times$ $(m+n)$ matrix, each element of which represents either the self-inductance of a vehicle or guideway coil or the mutual inductance between a vehicle coil and a guideway coil; and $[R]$ to be a diagonal $(m+n$ elements) matrix made up of the individual vehicle coil and guideway coil resistances. We can then write the system voltage equations in matrix form, on the basis of Kirchhoff's voltage law, as follows:

$$
[e]=[R][i]+\frac{d}{d t}\{[L][i]\}
$$

For a passive EDS guideway, the applied voltage term [e] is zero. However, each guideway coil contains induced voltages in connection with the vehicle's motions. Letting $v_{x}, v_{y}$, and $v_{z}$ be the 
speeds of the vehicle in the $x, y$, and $z$ directions, respectively, we can rewrite Equation 2.1 in terms of the induced voltages as

$$
[L] \frac{d}{d t}[i]+[R][i]=-v_{x}\left[G_{x}\right][i]-v_{y}\left[G_{y}\right][i]-v_{z}\left[G_{z}\right][i]
$$

where $\left[G_{x}\right]=\partial[L] / \partial x,\left[G_{y}\right]=\partial[L] / \partial y$, and $\left[G_{z}\right]=\partial[L] / \partial z$ are the derivatives of the mutual inductance between the vehicle-borne superconducting coils and the guideway coils with respect to the three displacements. Three time-dependent force components, $f_{x}, f_{y}$, and $f_{z}$, are obtained:

$$
\begin{aligned}
& f_{x}=\frac{1}{2}[i]^{T}\left[G_{x}\right][i], \\
& f_{y}=\frac{1}{2}[i]^{T}\left[G_{y}\right][i],
\end{aligned}
$$

and

$$
\mathrm{f}_{\mathrm{z}}=\frac{1}{2}[\mathrm{i}]^{\mathrm{T}}\left[\mathrm{G}_{\mathrm{z}}\right][\mathrm{i}]
$$

where $f_{x}$ is the force in the direction of vehicle motion, $f_{y}$ is the force in the horizontal direction, and $f_{z}$ is the force in the vertical direction. Note from Equation 2.2 that the currents induced in the guideway coils depend on the vehicle's dynamic motion. This implies that Equation 2.2 cannot be solved independently of the equations of motion of the vehicle. However, for the quasi-steadystate analysis focusing on the electromagnetic performance, the lateral and vertical speeds can be neglected and the longitudinal speed can be assumed to be constant. Since the mutual inductances and the derivatives of the mutual inductances in Equation 2.2 are functions of displacements that are, in turn, functions of time, the currents induced in the guideway coils can be solved by using an iterative process. For instance, by Euler's formula, multiplying the derivative of $i$ by the time increment and adding the resulting value to the previous $i$ will give a good approximation of the next $i$ at time $t$ :

$$
[i(t)]=[i(t-\Delta t)]+\frac{d[i]}{d t} \Delta t
$$

where the time increment can be expressed in terms of the longitudinal displacement increment divided by speed (i.e. $\Delta t=\Delta x / v_{x}$ ). Equation 2.2 can also be solved by using other numerical approaches, including the Runge-Kutta method. 
Time-averaged magnetic forces, $F_{x}, F_{y}$, and $F_{z}$, can be obtained by integrating Equations 2.3 through 2.5 over any desired time period:

$$
\begin{aligned}
& F_{x}=\frac{1}{T} \int_{0}^{T} \frac{1}{2}[i]^{T}\left[G_{x}\right][i] d t, \\
& F_{y}=\frac{1}{T} \int_{0}^{T} \frac{1}{2}[i]^{T}\left[G_{y}\right][i] d t,
\end{aligned}
$$

and

$$
F_{z}=\frac{1}{T} \int_{0}^{T} \frac{1}{2}[i]^{T}\left[G_{z}\right][i] d t .
$$

Assume that a figure-eight-shaped null-flux coil suspension system has $\mathrm{m}$ vehicle-borne superconducting coils interacting with $\mathrm{n}$ figure-eight-shaped null-flux coils. Since each figureeight-shaped null-flux coil consists of two cross-connected loops, $\mathbf{n}$ figure-eight-shaped null-flux coils are expected to contain $2 n$ loops. Name the upper row of the loops from 1 to $n$ and the bottom row of the loop as $n+1$ through $2 n$. In other words, the upper loop of the $i$ th figure-eightshaped null-flux coil is named as the ith loop, and the bottom loop of that is named as the $n+i$ th loop. Applying Equations 2.3 through 2.5 to the figure-eight-shaped null-flux coil system gives

$$
\begin{aligned}
& f_{x}=\sum_{i=1}^{n} \sum_{j=1}^{m} i_{i} I_{j}\left[\frac{\partial M_{i j}}{\partial x}-\frac{\partial M_{n+i, j}}{\partial x}\right] \\
& f_{y}=\sum_{i=1}^{n} \sum_{j=1}^{m} i_{i} I_{j}\left[\frac{\partial M_{i j}}{\partial y}-\frac{\partial M_{n+i, j}}{\partial y}\right],
\end{aligned}
$$

and

$$
f_{z}=\sum_{i=1}^{n} \sum_{j=1}^{m} i_{i} I_{j}\left[\frac{\partial M_{i j}}{\partial z}-\frac{\partial M_{n+i, j}}{\partial z}\right]
$$


where $\mathrm{M}_{\mathrm{ij}}$ is the mutual inductance between the jth superconducting coil and the ith loop of the null-flux coil and $I_{j}$ is the current in the $j$ th superconducting coil. The time-averaged magnetic forces can be obtained by using Equations 2.7 through 2.9, in terms of numerical expressions, as

$$
\begin{aligned}
& F_{x}=\frac{1}{k \Delta t} \sum_{l=1}^{k} \sum_{i=1}^{n} \sum_{j=1}^{m} i_{i}\left(t_{1}\right) I_{j}\left(t_{1}\right)\left[\frac{\partial M_{i j}\left(t_{1}\right)}{\partial x}-\frac{\partial M_{n+i, j}\left(t_{1}\right)}{\partial x}\right] \Delta t, \\
& F_{y}=\frac{1}{k \Delta t} \sum_{l=1}^{k} \sum_{i=1}^{n} \sum_{j=1}^{m} i_{i}\left(t_{l}\right) I_{j}\left(t_{l}\right)\left[\frac{\partial M_{i j}\left(t_{l}\right)}{\partial y}-\frac{\partial M_{n+1, j}\left(t_{1}\right)}{\partial y}\right] \Delta t,
\end{aligned}
$$

and

$$
F_{z}=\frac{1}{k \Delta t} \sum_{l=1}^{k} \sum_{i=1}^{n} \sum_{j=1}^{m} i_{i}\left(t_{1}\right) I_{j}\left(t_{1}\right)\left[\frac{\partial M_{i j}\left(t_{1}\right)}{\partial z}-\frac{\partial M_{n+i, j}\left(t_{1}\right)}{\partial z}\right] \Delta t,
$$

where the numerical integral is assumed to be conducted over a time period of $k \Delta t$ (i.e., $k$ time increments, each of value $\Delta t$ ).

\subsection{Determination of Circuit Parameters}

\subsubsection{Resistance and Inductance}

The resistance of a rectangular loop coil having sides of $s_{1} \times s_{2}$ (in meters), conductor cross-section per turn of $\mathbf{a} \times \mathbf{b}$ (in meters), and number of turns $N$ can be expressed as

$$
R=\frac{2\left(s_{1}+s_{2}\right) N}{a b \sigma},
$$

where $\sigma$ is the conductivity of the conductor. A figure-eight-shaped null-flux coil consists of two loop coils, so its resistance is twice the value given by Equation 2.16. 
The inductance of a rectangular loop coil (in henrys) is given by

$$
\begin{aligned}
L= & 10^{-6} \mathrm{~N}^{2}\left\{0.9209\left[\left(\mathrm{~s}_{1}+\mathrm{s}_{1}\right) \log _{10} \frac{2 \mathrm{~s}_{1} \mathrm{~s}_{1}}{\mathrm{a}+\mathrm{b}}-\mathrm{s}_{1} \log _{10}\left(\mathrm{~s}_{1}+\sqrt{\mathrm{s}_{1}^{2}+\mathrm{s}_{2}^{2}}\right)-\mathrm{s}_{2} \log _{10}\left(\mathrm{~s}_{2}+\sqrt{\mathrm{s}_{1}^{2}+\mathrm{s}_{2}^{2}}\right)\right]\right. \\
& \left.+0.4\left[2 \sqrt{\mathrm{s}_{1}^{2}+\mathrm{s}_{2}^{2}}-\frac{\mathrm{s}_{1}+\mathrm{s}_{2}}{2}+0.447(\mathrm{a}+\mathrm{b})\right]\right\}
\end{aligned}
$$

with all dimensions given in meters. Note that the resistance and inductance of the loop coils are constants, independent of the vehicle displacements.

\subsubsection{Determination of Mutual Inductance}

The most important parameters for the computer modeling are the mutual inductances between the guideway coils and the vehicle coils. These parameters are functions of the vehicle's displacements and need to be calculated at every time increment.

The fundamental relation for determining the mutual inductance between any two conductors defined by $C_{1}$ and $C_{2}$ is given by the Neumann formula

$$
M=\frac{\mu_{0}}{4 \pi} \int_{\mathfrak{c}_{1}} \int_{\mathrm{c}_{1}} \frac{d x d x^{\prime}+d y^{\prime} y^{\prime}+d z d z^{\prime}}{\left[\left(x-x^{\prime}\right)^{2}+\left(y-y^{\prime}\right)^{2}+\left(z-z^{\prime}\right)^{2}\right]^{1 / 2}},
$$

where $x, y$, and $z$, and $x^{\prime}, y^{\prime}$, and $z^{\prime}$ are the integral variables for conductors $C_{1}$ and $C_{2}$, respectively. Equation 2.18 can be generally applied to any geometry of the conductors. However, in some cases, it is difficult to obtain an analytical expression with Equation 2.18; therefore, numerical solution may become necessary. The simplest model for the determination of magnetic forces between the guideway coils and the vehicle coils is to treat the two coils as four finite-length conductors, as shown in Figure 2.1. The mutual inductance between the two 


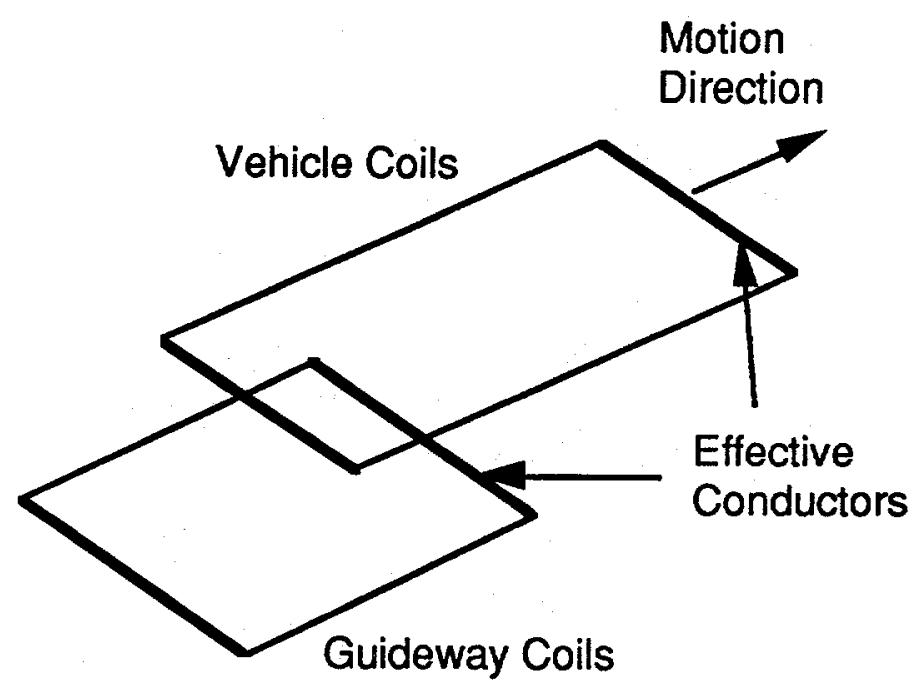

FIGURE 2.1 Interaction between Two Coils

Simplified as the Interaction between Four Effective Conductors

filaments, as shown in Figure 2. 2, with length 1 and $\mathrm{m}$, respectively, separated by a distance of d and with an overlapping length of $\delta$, is 6

$$
\begin{aligned}
M= & \frac{\mu_{0}}{4 \pi}\left[\alpha \sinh ^{-1} \frac{\alpha}{d}-\beta \sinh ^{-1} \frac{\beta}{d}-\gamma \beta \sinh ^{-1} \frac{\gamma}{d}+\delta \beta \sinh ^{-1} \frac{\delta}{d}\right. \\
& \left.-\sqrt{\alpha^{2}+d^{2}}+\sqrt{\beta^{2}+d^{2}}+\sqrt{\gamma^{2}+d^{2}}+\sqrt{\delta^{2}+d^{2}}\right]
\end{aligned}
$$

where

$$
\begin{aligned}
& \alpha=1+\mathrm{m}-\delta, \\
& \beta=1-\delta, \text { and } \\
& \gamma=\mathrm{m}-\delta
\end{aligned}
$$

Note that in the computer modeling, the variables $1, \mathrm{~m}, \mathrm{~d}$, and $\delta$ should be expressed in terms of $\mathrm{x}$, $y$, and $z$, as shown in Figure 2.2, because $d$ and $\delta$ are functions of displacements. 


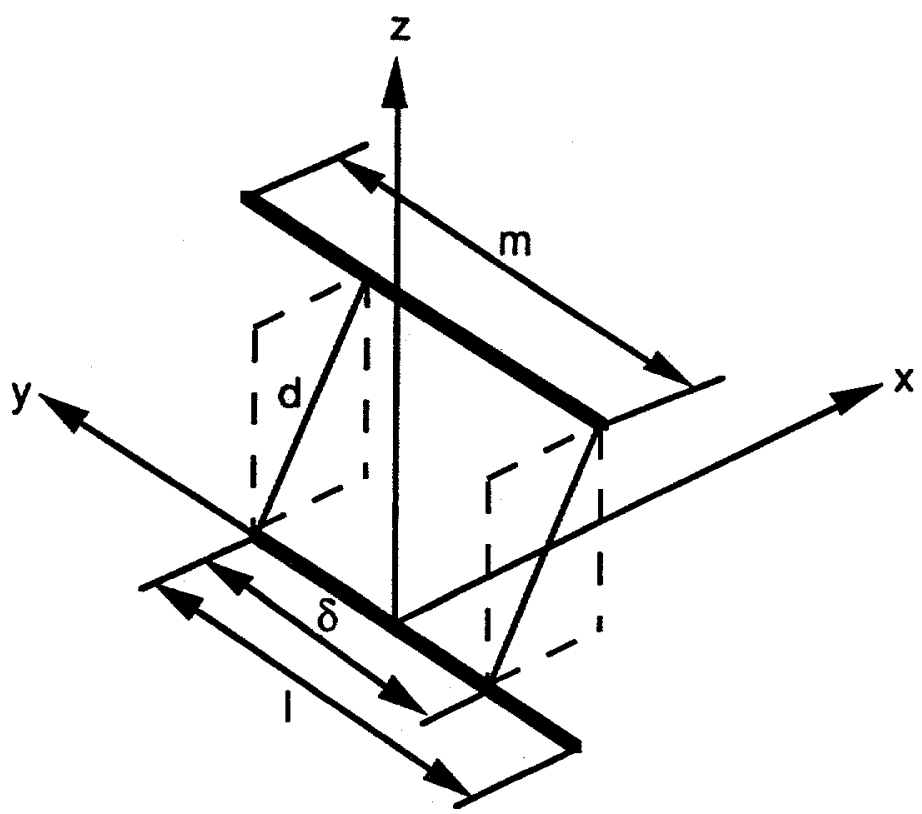

FIGURE 2.2 Determination of Mutual Inductance between Two Finite-Length Conductors

On the other hand, one can determine the mutual inductance between the two rectangular coils precisely, instead of using the two conductor formulas described by Equation 2.19. A general relation for the mutual inductance between the two rectangular coils, referring to Figure 2.3, is given $\mathrm{by}^{7}$

$$
M=\frac{\mu_{0}}{4 \pi} \sum_{i=1}^{4} \sum_{i=1}^{4}(-1)^{i+j} \ln \left[\Psi\left(x_{j}, y_{j}, 0 ; x_{i}^{\prime}, y_{i}^{\prime}, z_{0}\right)+\Psi\left(y_{j}, x_{j}, 0 ; y_{i}^{\prime}, x_{i}^{\prime}, z_{0}\right)\right]
$$

where

$$
\begin{aligned}
\Psi\left(x, y, z, x^{\prime}, y^{\prime}, z^{\prime}\right)= & -\left(x-x^{\prime}\right) \ln \left[\left(x-x^{\prime}\right)+\sqrt{\left(x-x^{\prime}\right)^{2}+\left(y-y^{\prime}\right)^{2}+\left(z-z^{\prime}\right)^{2}}\right] \\
& +\sqrt{\left(x-x^{\prime}\right)^{2}+\left(y-y^{\prime}\right)^{2}+\left(z-z^{\prime}\right)^{2}} .
\end{aligned}
$$

Equation 2.20 is very effective and useful for most coil-type guideways, including the loop coil guideway and the figure-eight-shaped null-flux coil guideway. 


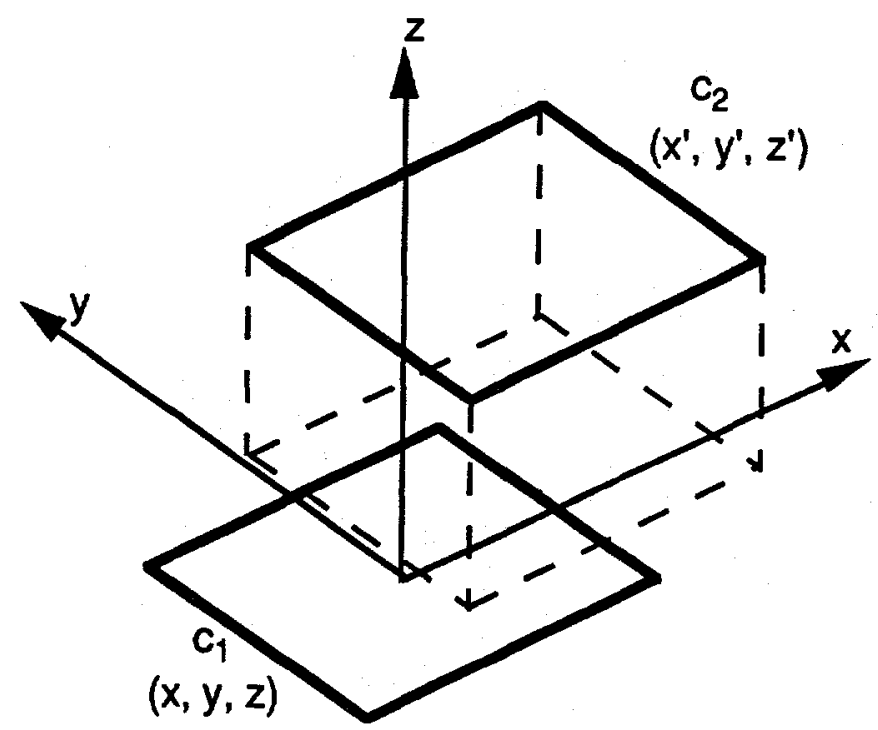

FIGURE 2.3 Determination of Mutual Inductance between Two Rectangular Coils

\subsection{Introduction to the Computer Code}

Several computer codes have been developed, on the basis of the dynamic circuit model, for the computation of magnetic forces for several different coil-type guideways. Each code may differ slightly from others. However, the main programs of these codes are very similar; Figure 2.4 illustrates a flow chart of the main programs. The program basically consists of several parts: input data process, circuit parameter computations, solution of the system equations, time-dependent and time-averaged force computations, and the output data process. The input data include the dimensions of guideway coils and superconducting coils, material specifications, vehicle speed, superconducting coil currents, and other program parameters. The circuit parameters, stored in matrix form, include the resistances and inductances of the guideway coils, the mutual inductances between the vehicle coils and the guideway coils, and the derivatives of the mutual inductances. Of these parameters, the resistances and inductances are constant and are calculated at the beginning of the program. However, the mutual inductances and their derivatives are functions of displacements and are calculated at every time increment. The currents induced in the guideway coils are then obtained by solving Equation 2.1, and the time-dependent magnetic forces and the time-averaged magnetic forces are determined from using Equations 2.10 through 2.12 and Equations 2.13 through 2.15 , respectively. The output data contain the simulation results stored in several files that can be used as input by some graphical programs.

\subsection{Numerical Example}

To demonstrate the capabilities of the dynamic circuit model and to help the reader understand the performance of a maglev system using the null-flux suspension concept, an EDS 


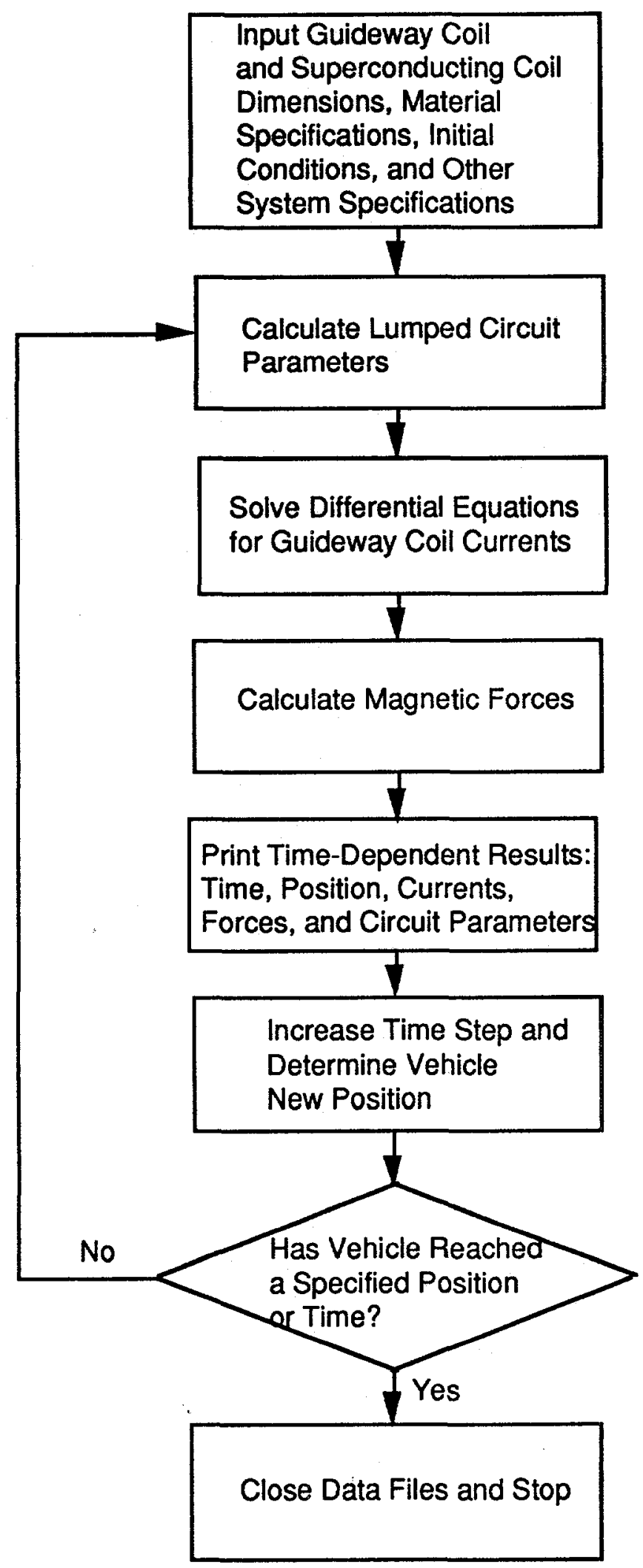

FIGURE 2.4 Block Diagram of the Main Program 
maglev system, developed in Japan, using side-wall null-flux suspension, is used as a numerical example. Figure 2.5 shows a cross-sectional view of the system on which the computer simulations were based; Table 1 gives the corresponding dimensions. The force-velocity characteristic curve is shown in Figure 2.6, where one can see that the lift force on a 700-kA-T $S C M$ is $15 \mathrm{kN}$ at $250 \mathrm{~km} / \mathrm{h}$ and $16 \mathrm{kN}$ at $500 \mathrm{~km} / \mathrm{h}$, with a vertical displacement of $3 \mathrm{~cm}$. The drag force approaches a maximum value of $0.25 \mathrm{kN}$ at about $20 \mathrm{~km} / \mathrm{h}$, and it is only about $0.064 \mathrm{kN}$ at $500 \mathrm{~km} / \mathrm{h}$, resulting in a lift-to-drag ratio of over 250 . Figure 2.7 shows the lift and lift-to-drag ratio as functions of vertical displacement. A maximum lift force of $41.5 \mathrm{kN}$ appears at a vertical displacement of $11.5 \mathrm{~cm}$; this gives a safety factor, defined as the maximum lift over full-load lift $(3 \mathrm{~cm})$, of 2.5 to 3.5 . It can be concluded from this computation that a vehicle having twelve SCMs is able to lift $180 \mathrm{kN}$ at a vertical displacement of $3 \mathrm{~cm}$ for vehicle speeds higher than $250 \mathrm{~km} / \mathrm{h}$, as shown in Figures 2.6 and 2.7. At lower speeds, the vertical displacement is expected to increase in order to achieve the same amount of lift force. As shown in Figure 2.7, at a $3 \mathrm{~cm}$ vertical displacement, for example, the lift-to-drag ratio is 280 , corresponding to $1 \mathrm{kN}$ of drag force with $280 \mathrm{kN}$ of lift force. The program can also calculate the time-dependent magnetic force pulsation resulting from the discrete coil guideway, as shown in Figure 2.8. This information is useful for the design of superconducting magnets and other mechanical components.

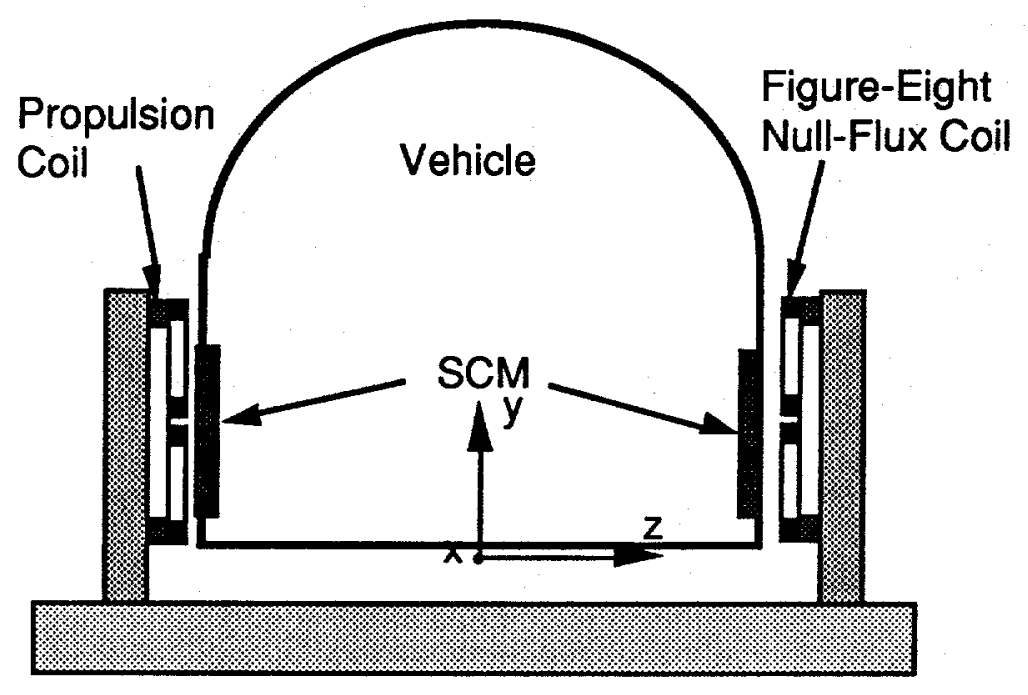

FIGURE 2.5 Side-Wall Suspension System (cross section) 
TABLE 2.1 Data Used for Computer Simulation

\begin{tabular}{lc}
\hline \multicolumn{1}{c}{ Parameter } & Value \\
& \\
\hline & \\
Vehicle & 17 \\
Total weight (tonnes) & 500 \\
Maximum velocity (km/h) & 3.7 \\
Height (m) & 3 \\
Width (m) & 12 \\
Number of SCMs & \\
& \\
Superconducting magnet (SCM) & 1.7 \\
Length (x-direction) (m) & 0.5 \\
Height (z-direction) (m) & 0.4 \\
Gap between magnets (m) & 700 \\
Current (NI) (kA-T) & \\
Ground null-flux coil & 0.55 \\
Length (x-direction) (m) & 0.31 \\
Height (z-direction) (m) & 0.12 \\
Distance between upper and lower & \\
$\quad$ loops (m) & 0.15 \\
Distance between null-flux coils (m) & 36 \\
Number of turns & 1 \\
Al conductor (cross section) (cm & \\
\hline
\end{tabular}




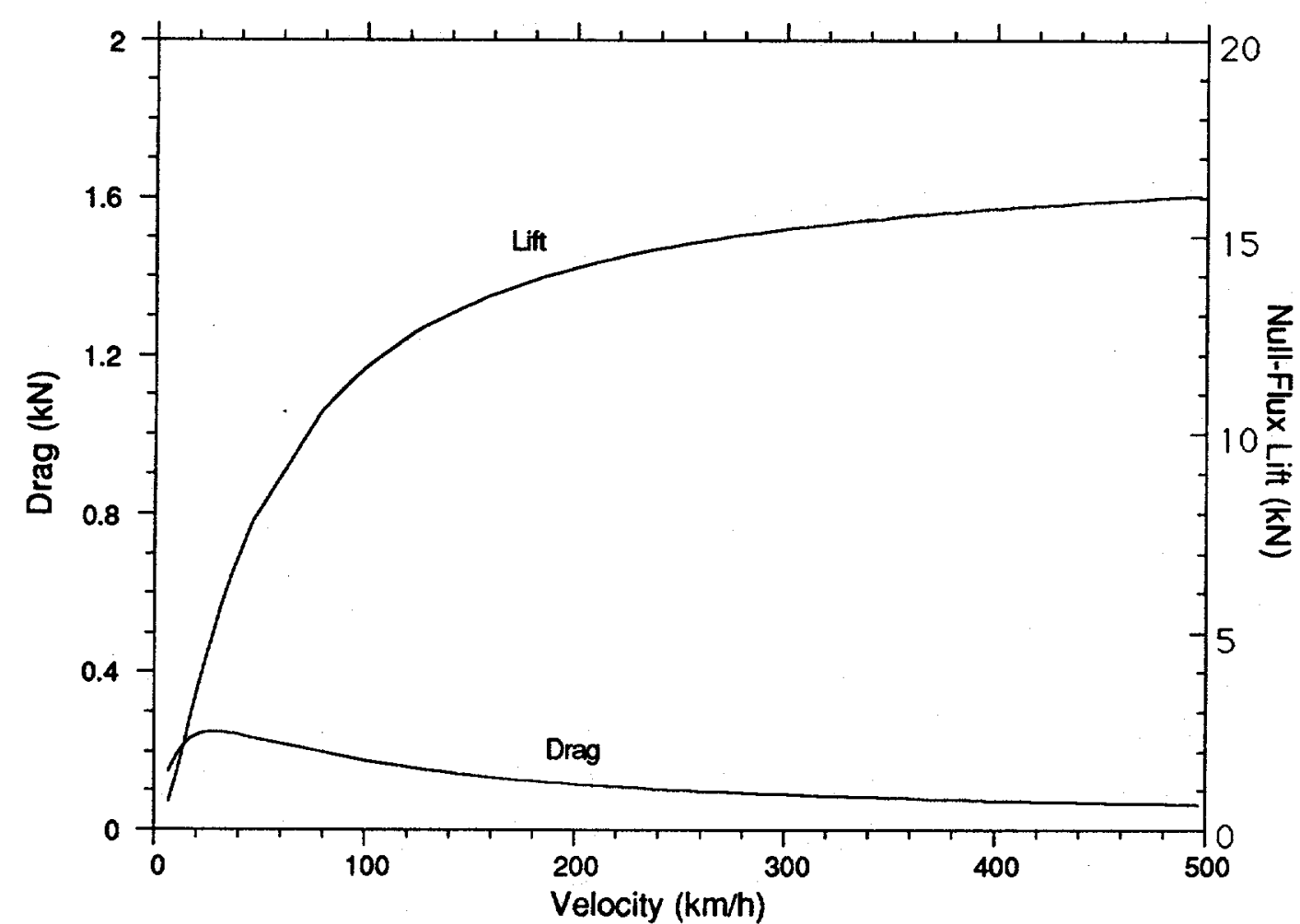

FIGURE 2.6 Null-Flux Lift and Drag Force as Functions of Vehicle Velocity (air gap $=20 \mathrm{~cm}$, vertical displacement $=3 \mathrm{~cm}, S C M$ current $=700 \mathrm{kA}-\mathrm{T}$ )

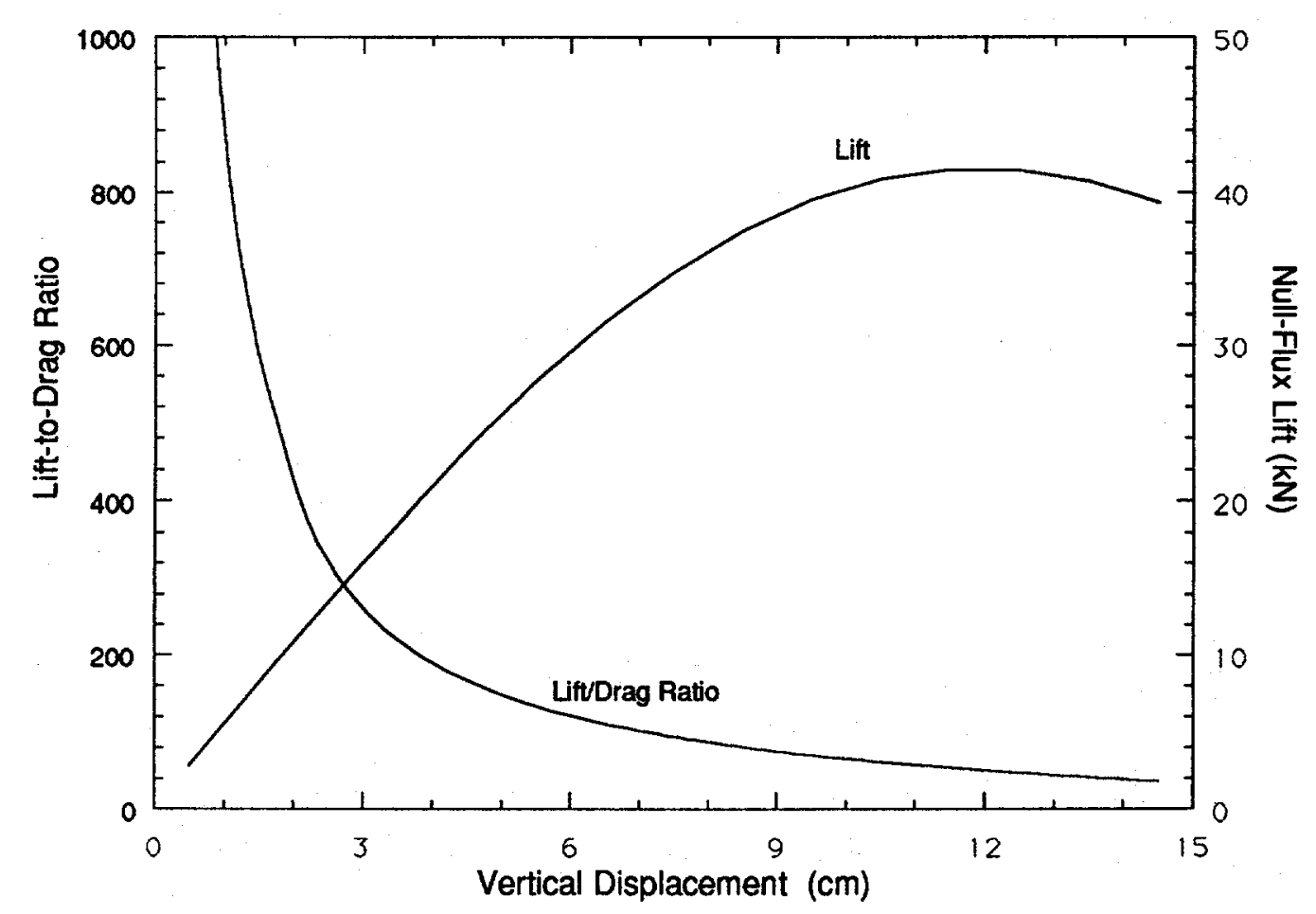

FIGURE 2.7 Null-Flux Lift and Lift-to-Drag Ratio per SCM as Functions of Vertical Displacement 


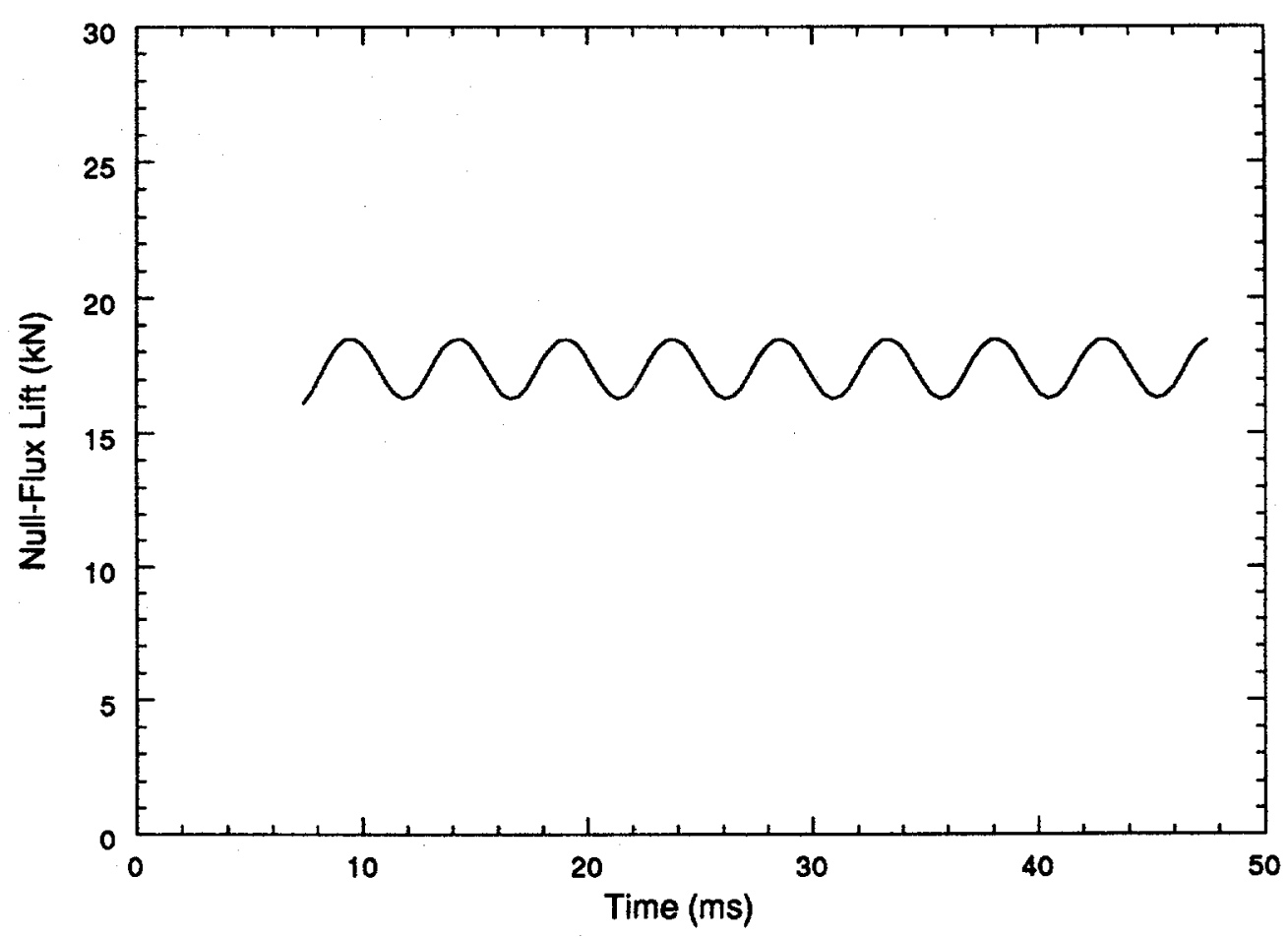

FIGURE 2.8 Null-Flux Lift as a Function of Time 


\section{Experimental Verification}

\subsection{Experimental Apparatus}

To verify the computer code based on the dynamic circuit theory, an experimental device was developed for the measurement of magnetic force of the figure-eight-shaped null-flux coils, as shown in Figure 3.1. A schematic diagram of the experimental null-flux apparatus is shown in Figure 3.2. The apparatus consists of a PVC drum, with an inside diameter of $0.24 \mathrm{~m}$; figureeight-shaped coils; magnet and force transducer; and a network/spectrum analyzer. The figureeight-shaped null-flux coils were mounted on the inside surface of a PVC drum (Figures 3.3 and 3.4). The drum was then statically balanced to insure safety. The rotational speed of the drum could be varied from 0 to $1800 \mathrm{rpm}$ with a maximum linear speed of $22.6 \mathrm{~m} / \mathrm{s}$. Speed is calculated at the center of the coil. A NdFeB permanent magnet $(25.4 \mathrm{~mm}$ square by $3.175 \mathrm{~mm}$ thick ) was attached to a 9251A tri-axial piezoelectric force transducer and both were mounted on a support bar suspended over the moving coils. The design of the bar minimized resonance effects and cross-talk interference with the transducer. The support bar was cantilevered from a separate, massive concrete block to minimize coupling between the support bar and the drum. The coil drum is mounted on a milling machine table for three-dimensional positioning of the drum and coils.

As the drum rotates clockwise, there is an induced voltage in the coils due to the relative motion of the magnet-coil system. This voltage creates current flow (except at equilibrium position), which results in a secondary magnetic field in opposition to the change in flux due to relative motion. Interactions between the piezoelectric crystals within the transducer create a charge, which is sent from the transducer to an Endevco 2730 charge amplifier. The charge amplifier converts the charge to voltage, and relays this $\mathrm{V}_{\text {out }}$ to a Hewlett-Packard (HP) Vectra Network/Spectrum Analyzer. Through a math conversion subroutine within the HP unit, this voltage history can be transformed into a force-time history.

\subsection{Dynamic Characteristics of Single-Turn Figure-Eight- Shaped Null-Flux Coils}

The first experiment was conducted with five different configurations of figure-eightshaped null-flux coils mounted sequentially around the inner surface of the drum. Figure 3.5 shows the positions of the five coils inside the PVC drum. All figure-eight-shaped null-flux coils are made with two single-turn No. 12-copper-magnet-wire loops. Figure 3.6 shows the five single-turn figure-eight-shaped null-flux coil configurations.

- Configuration A: a figure-eight-shaped null-flux coil with two 25.4-mm-square loops. 


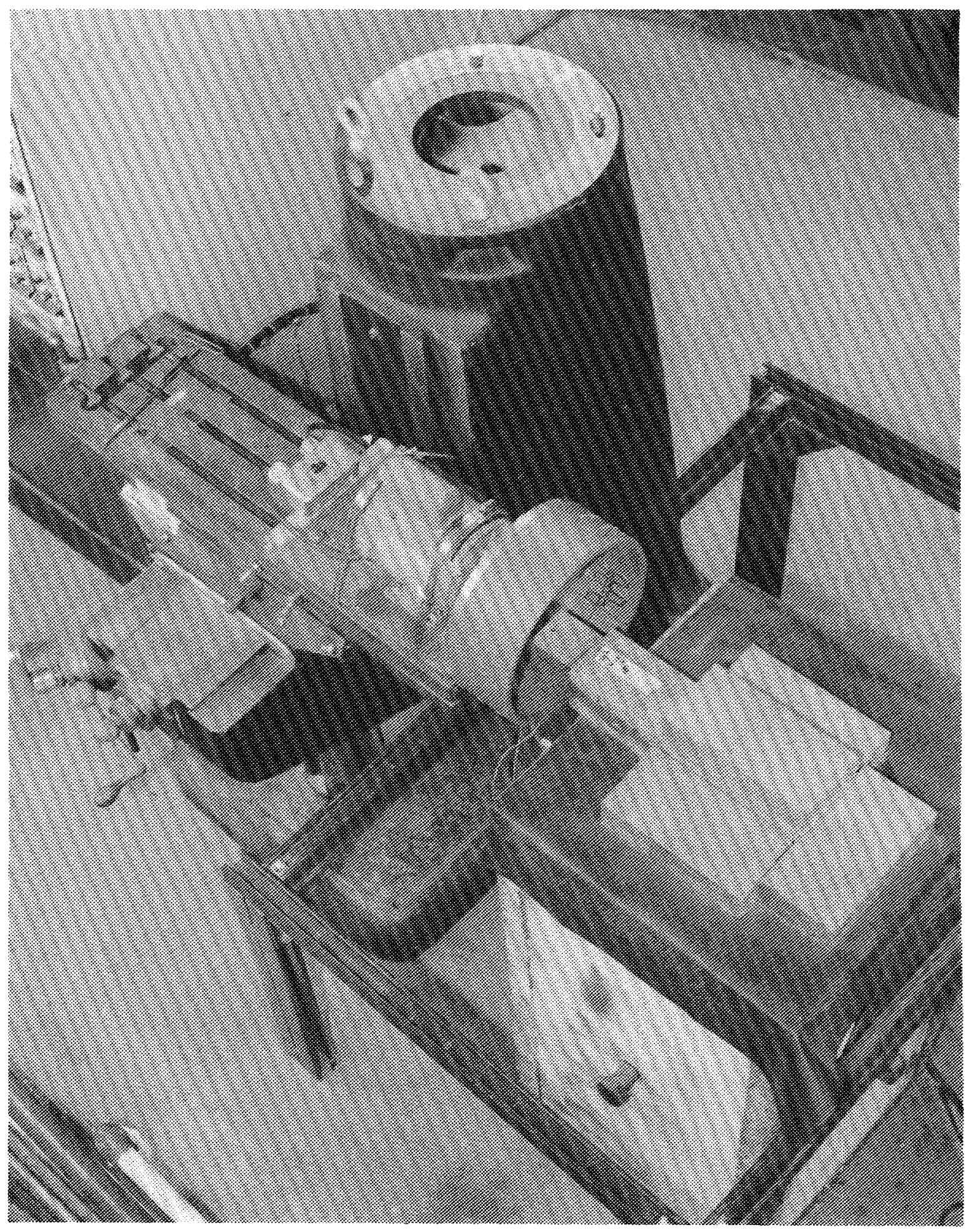

FIGURE 3.1 Photograph of the Experimental Null-Flux Apparatus 
NULL-FLUX APPARATUS

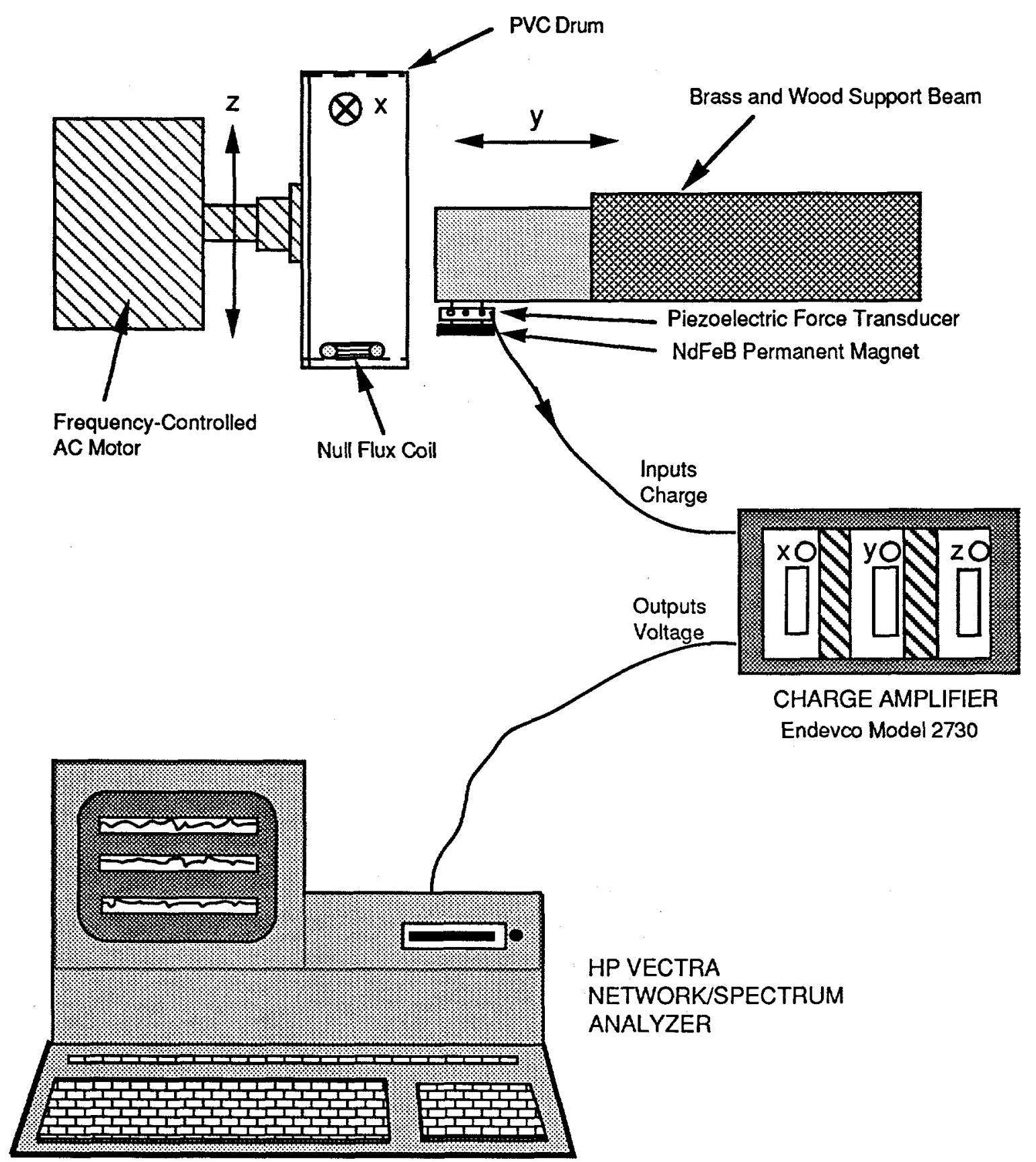

FIGURE 3.2 Schematic of the Experimental Null-Flux Apparatus 


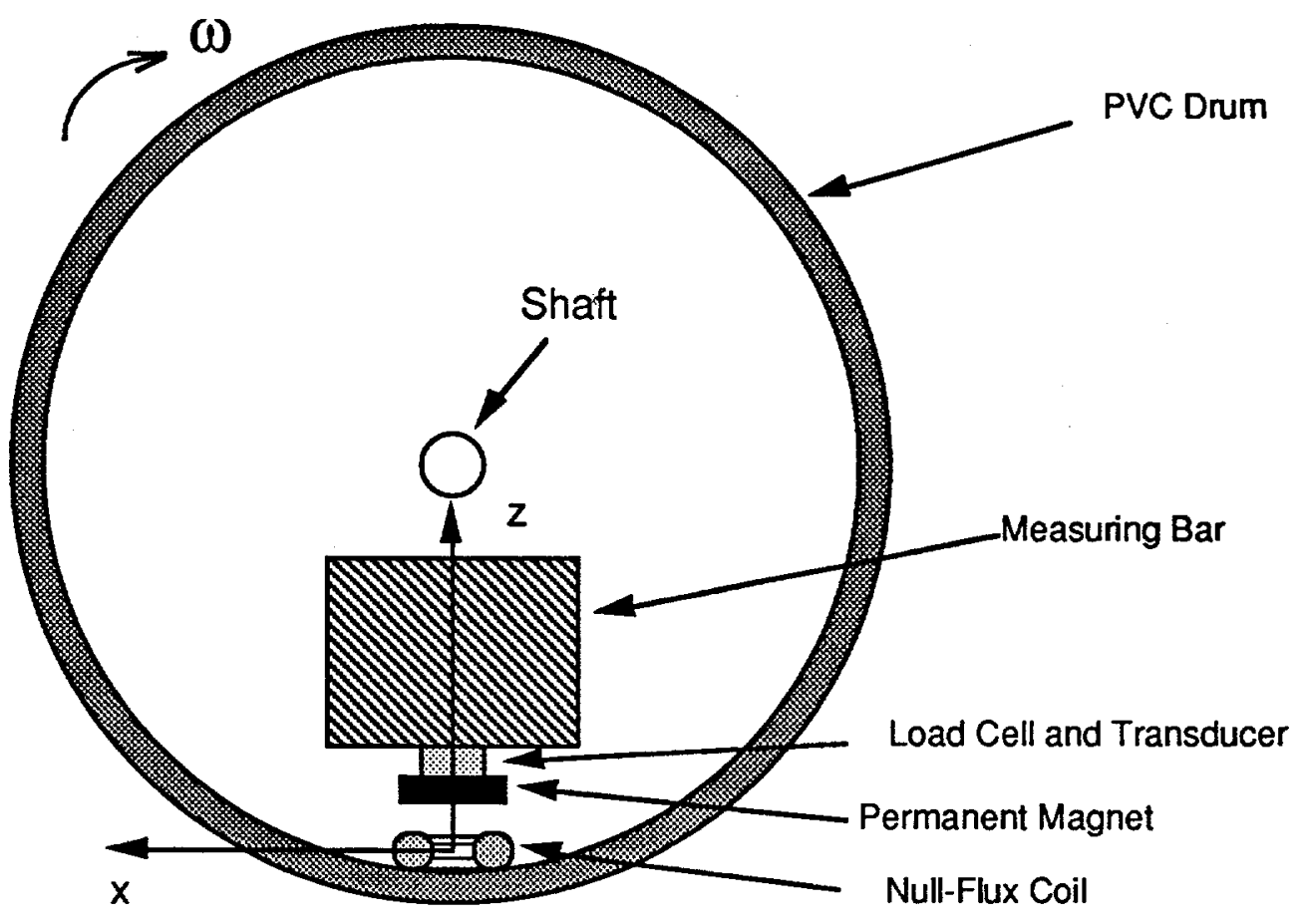

FIGURE 3.3 Experimental Null-Flux Apparatus (front view)

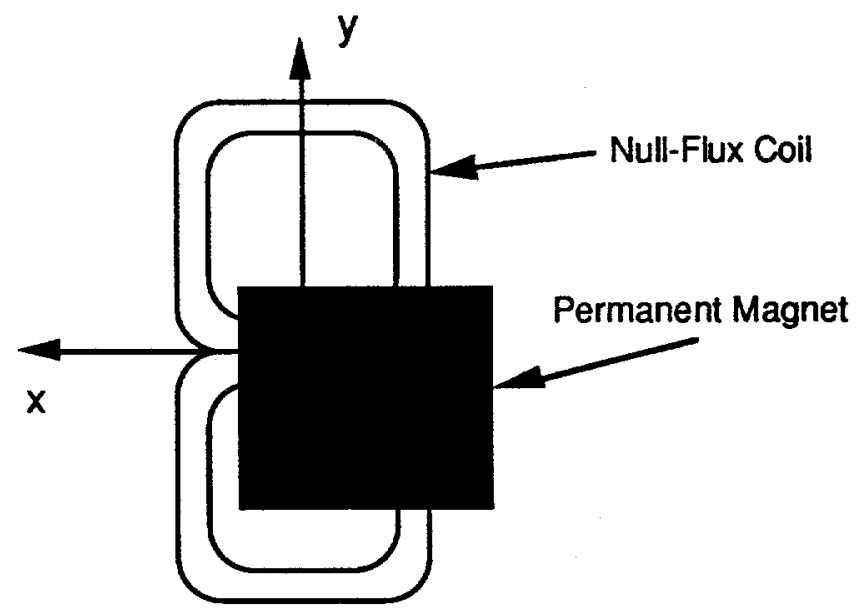

FIGURE 3.4 Magnet Orientation (top view) 


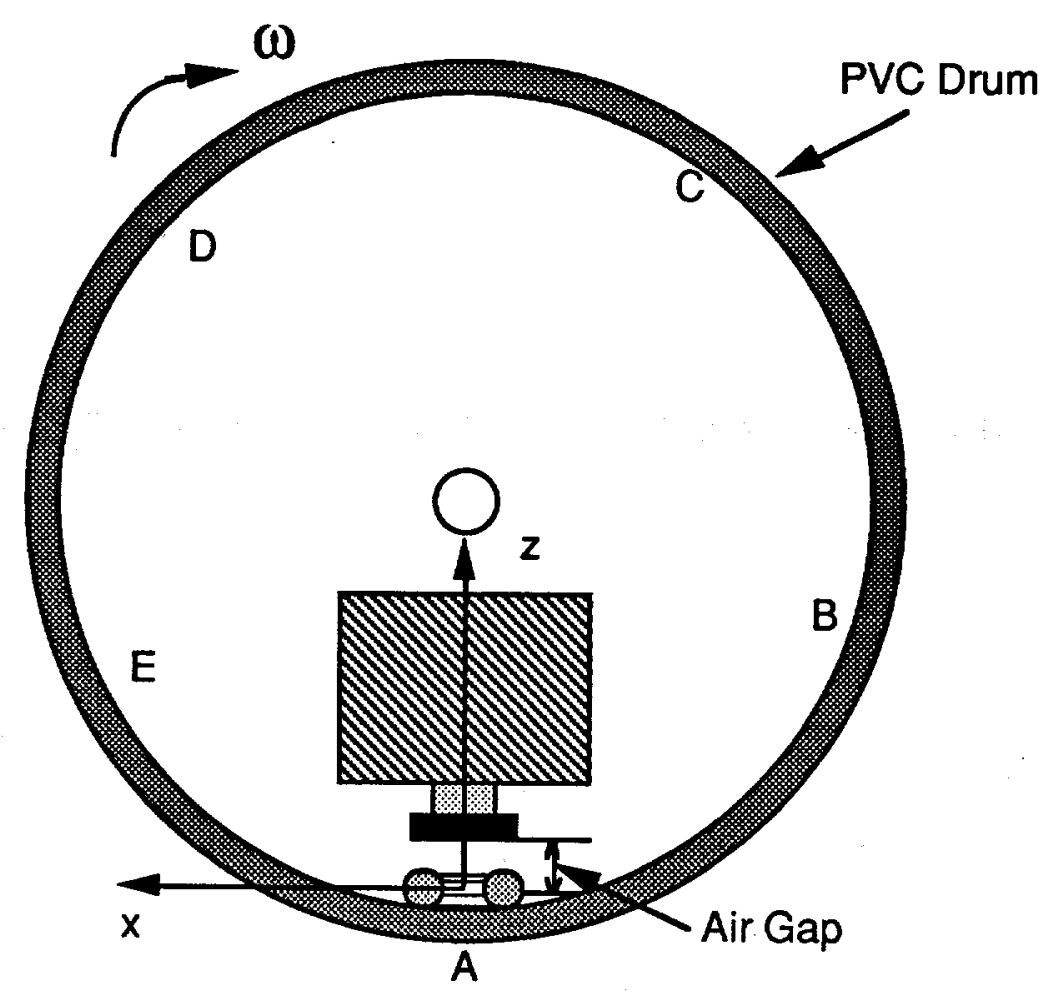

FIGURE 3.5 Null-Flux Coil Configuration Positions in Drum (cross section)

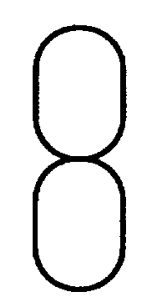

Configuration A

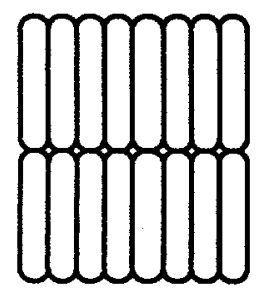

Configuration

B

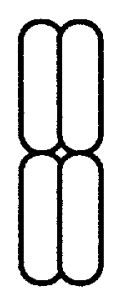

Configuration C

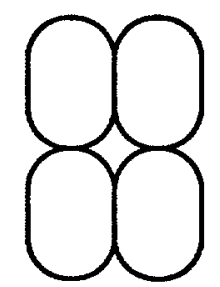

Configuration D

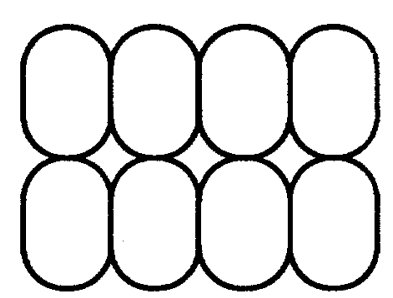

Configuration E

FIGURE 3.6 Single-Turn Figure-Eight-Shaped Null-Flux Coil Configurations (top view) 
- Configuration B: eight figure-eight-shaped coils with loop dimensions of $25.4 \times 6.35 \mathrm{~mm}$.

- Configuration $\mathrm{C}$ : four figure-eight-shaped coils with loop dimensions of $25.4 \times 12.7 \mathrm{~mm}$.

- Configuration D: two figure-eight-shaped coils, each coil has two 25.4-mmsquare loops.

- Configuration E: four figure-eight-shaped coils, each coil with 25.4-mmsquare loops.

Three dimensional magnetic forces were measured and calculated for different air gaps (distance between the bottom surface of the magnet and the center plane of the coils) in the $z$ axis direction, and the horizontal displacement (y) between the magnet and the figure-eight-shaped coils. Figure 3.7 shows the measured lift, guidance, and drag forces acting on the permanent magnet at a speed of $22.6 \mathrm{~m} / \mathrm{s}$, as a function of time (horizontal offset $y=10.2 \mathrm{~mm}$ and air gap $\mathrm{z}=4 \mathrm{~mm}$ ). The horizontal displacement y coordinate is zero when the magnet is centered over the figure-eight-shaped null-flux coils. This is defined as the null-flux equilibrium.

Note that each time-history (as shown in Figure 3.6) shows magnetic forces for five different coil configurations (A, B, C, D, and E). The top signal represents the null-flux lift force (y) (parallel to the length of the coil), the middle signal gives the drag force (x) (opposing the motion), and the bottom signal shows the guidance signal (z) (perpendicular to the coil).

The drag force due to a single null-flux coil (configuration A) consists of two impulses that occur when the magnet approaches and departs from the center of the coil. The superposition of similar pairs of impulses for configurations $B$ through $E$ result in waveforms $B$ to $E$ in Figure 3.7. The impulse overlap is caused by the fact there is no space between the parallel-arranged null-flux coils for coil configurations $B$ through $\mathrm{E}$. The lift ( $\mathrm{y}$ directed) and guidance forces ( $\mathrm{z}$ directed) alternate their sign from positive to negative, or from negative to positive, as the magnet crosses the null-flux coils. This phenomenon is caused by the transition time between the magnet and the null-flux coils being no longer than the time constant of the null-flux coils. 5

A computer simulation of calculated magnetic forces as a function of time for null-flux coil configuration A, using one of the COILGDWY codes, is shown in Figure 3.8. A comparison of the measured forces (Figure 3.7) and the computer calculated forecast (Figure 3.8) shows that a quantitative agreement was achieved between the calculated and measured results. By using the same COILGDWY computer code, one can also predict the characteristics of the null-flux coil configurations at a speed of $100 \mathrm{~m} / \mathrm{s}$, as show in Figure 3.9, where the lift force has larger negative impulses and the guidance force has larger positive impulses. That is, the time-averaged lift and guidance forces increase as the velocity increases. Figure 3.10 shows the time-averaged 


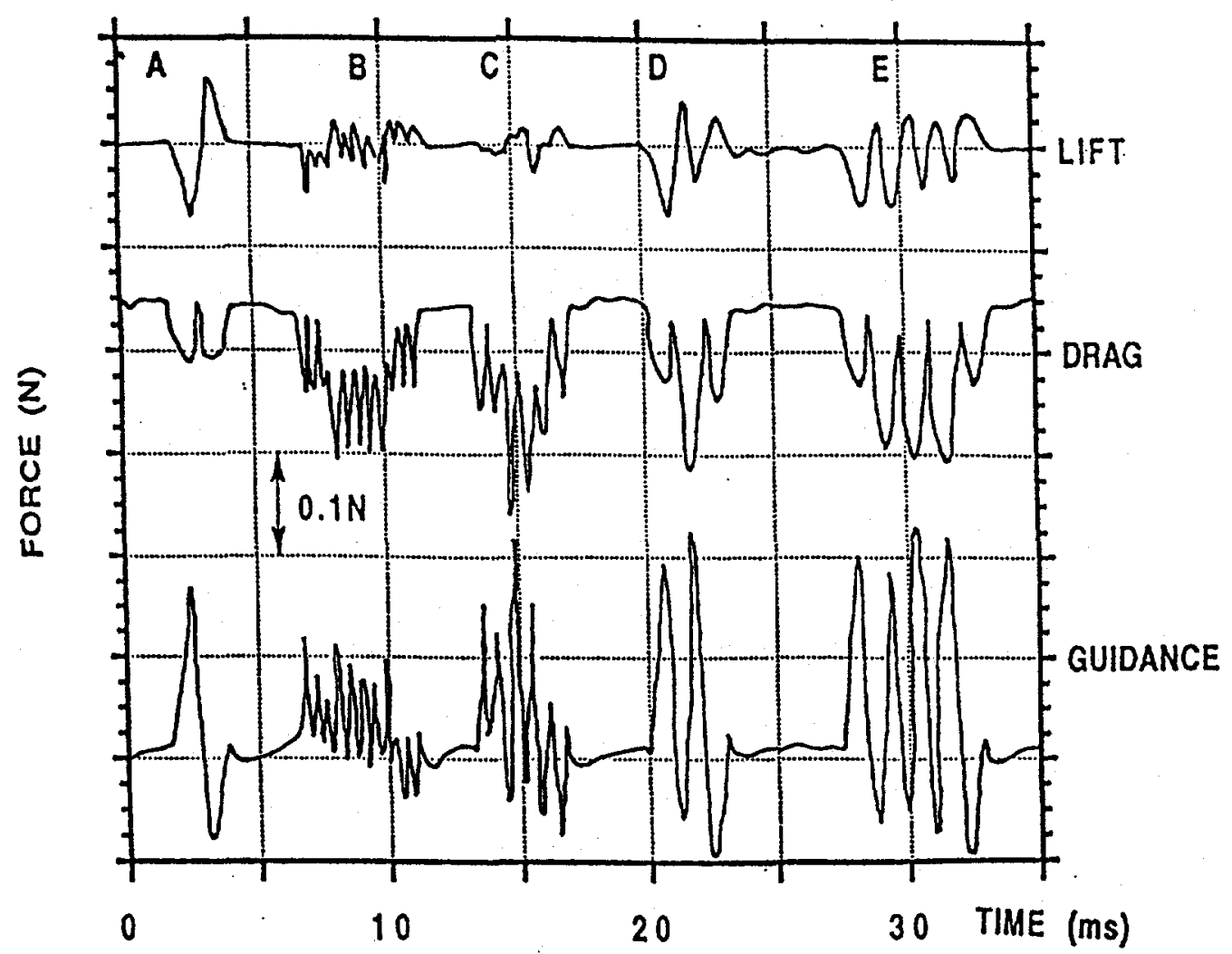

FIGURE 3.7 Measured Magnetic Forces as a Function of Time for Null-Flux Coil Configurations A through $E$ (velocity $=22.6 \mathrm{~m} / \mathrm{s}$, horizontal displacement $y=10.2 \mathrm{~mm}$, air gap $z=4 \mathrm{~mm}$ )

magnetic forces corresponding to the null-flux coil configuration $\mathrm{A}$, where the lift force is less than the drag force (lift-to-drag ratio is less than one) for speeds lower than about $55 \mathrm{~m} / \mathrm{s}$, and greater than the drag force (lift-to-drag ratio greater than one) for speeds higher than about $55 \mathrm{~m} / \mathrm{s}$. This, of course, is not acceptable to a normal maglev system where the null-flux lift is usually much lager than the drag force.

Figures 3.11 and 3.12 show the time-averaged magnetic force dependence on horizontal displacement (y) for speeds of $22.6 \mathrm{~m} / \mathrm{s}$ and $100 \mathrm{~m} / \mathrm{s}$, respectively. Note that at high speed $(100 \mathrm{~m} / \mathrm{s})$, the null-flux lift force characteristics improve. It can be concluded from these results that the characteristic ratio (defined as the time constant of the null-flux divided by the time required by the magnet to transverse across one null-flux coil distance) of the null-flux coil used in this experiment is too small. To improve the lift-to-drag ratio, it is necessary to either increase the speed or increase the time constant of the null-flux coils. 


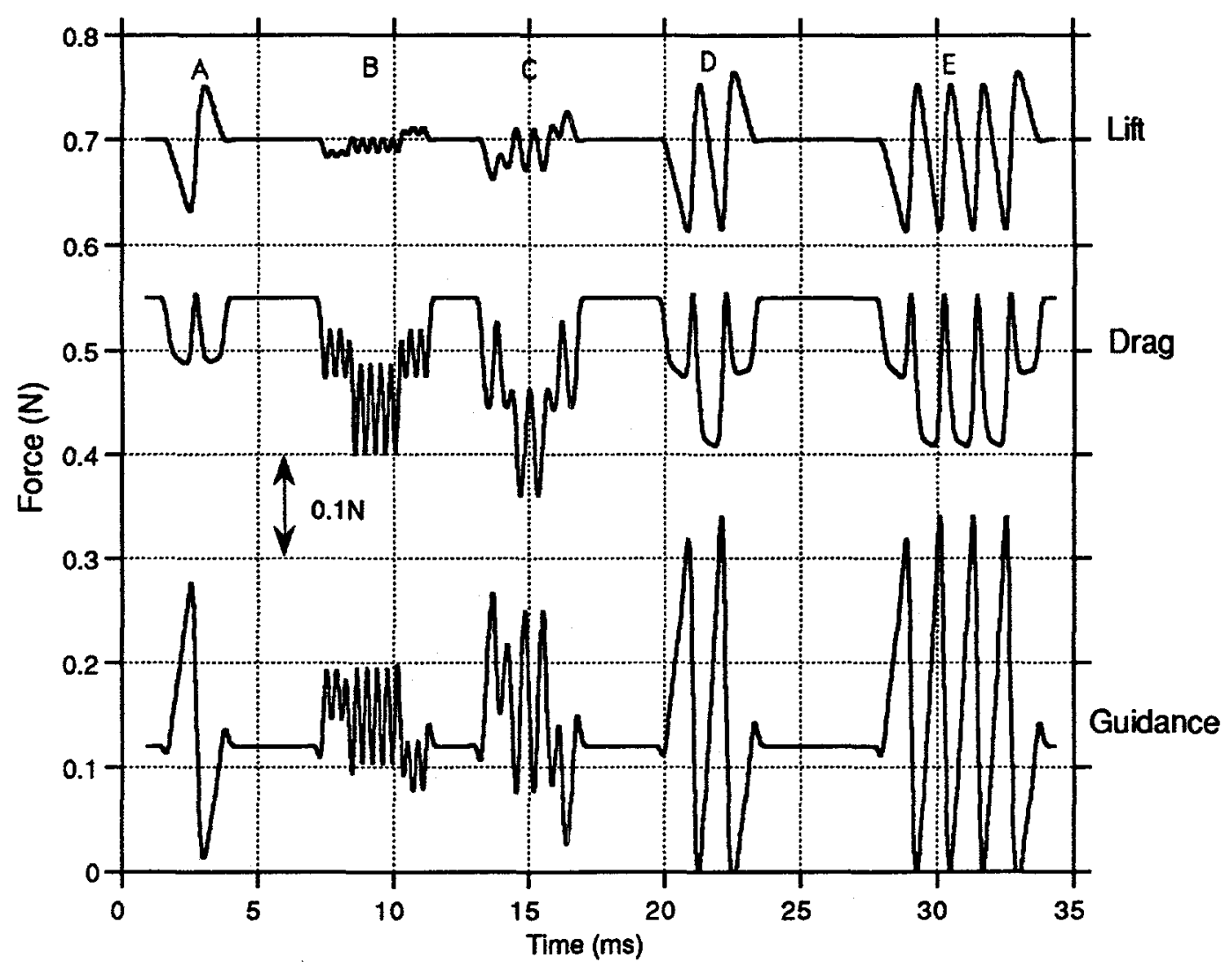

FIGURE 3.8 Computer-Calculated Magnetic Forces Acting on Various FigureEight-Shaped Null-Flux Coils, Using the Same Parameters as in Figure 3.7

\subsection{Dynamic Force Characteristics}

As observed from both the computer simulations and the experimental results discussed in the previous section, the time-dependent magnetic force waveforms involve both positive and negative impulses as the magnet passes over the stationary guideway coils. The time-averaged magnetic forces are determined by the areas enclosed by the positive and negative impulses. The objective of a null-flux coil suspension system design is to obtain a greater time-averaged lift force, or to increase the difference between the two impulses. Thus, it is essential to understand the mechanism of the dynamic force waveforms. Figures 3.13 through 3.15 show different models that can explain the characteristics of the dynamic forces. Figure 3.13 (a) shows a permanent magnet (with the north pole facing down and the south pole facing up) approaching a stationary guideway coil, which senses a downward increasing flux. According to the Lenz's law, an induced current flowing in the coil produces a flux apposing the increasing flux caused by the moving magnet. At this point, the system is analogous to two permanent magnets with the north poles facing each other. The repulsive force, $\mathbf{F}$, between the two can be resolved into two components: the drag force component, $F_{X}$, which is opposite to the motion direction, and the lift force component, $F_{\mathrm{z}}$, which is repulsive force between the magnet and the coil. Their timedependent waveforms are shown in solid lines in Figure 3.13 (b) and 3.13 (c), respectively. 


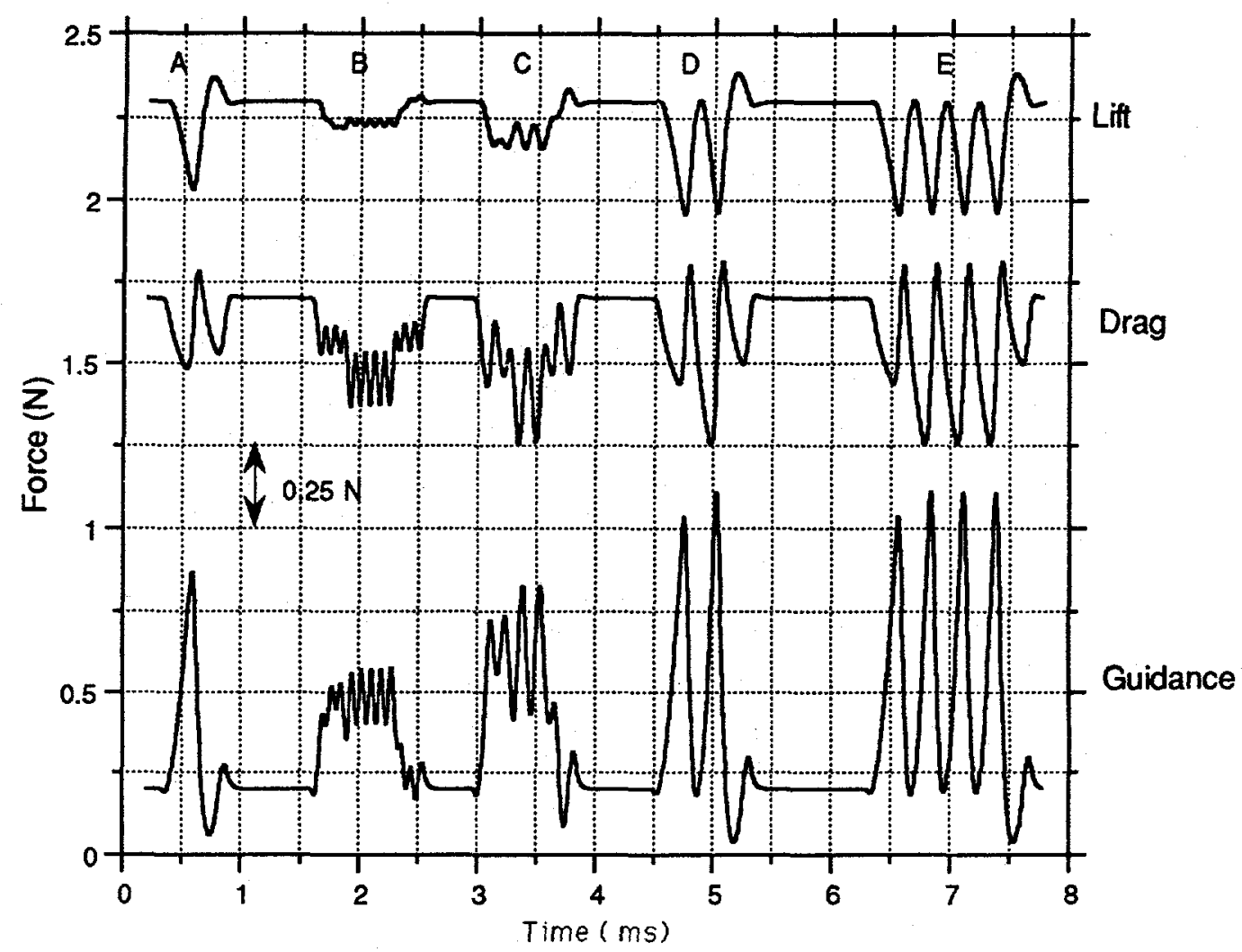

FIGURE 3.9 Computer-Calculated Magnetic Forces as a Function of Time for Null-Flux Coil Configurations A through $E$ at a Speed of $100 \mathrm{~m} / \mathrm{s}$

Figure 3.14 shows a permanent magnet moving away from a stationary guideway coil. In this case, the total flux linking the coil decreases as the magnet moves away from the coil. Again, according to the Lenz's law, a current induced in the coil prevents the total flux from decreasing if the time constant of the coil is shorter than the traverse time of the magnet. Note in Figure 3.14 (a) that the current induced in the coil changes its direction, as compared to Figure 3.13 (a). At this point, the system is analogous to two magnets with the north pole facing to the south pole. An attractive force, $\mathbf{F}$, between the magnet and the coil is developed. This force can be resolved into two components: the vertically attractive force, $F_{z}$, and the longitudinal force, $F_{x}$. The corresponding time-dependent force waveforms are shown in solid lines in Figure 3.14 (b) and (c), respectively. Note that the second impulse is generally smaller than the first one because of the time constant of the stationary guideway coil. Thus, the time-averaged lift force is positive and the time-averaged drag is always negative.

However, the second impulses of the lift and drag forces become positive as the time constant of the coil increases, or the speed of the magnet increases, as shown in Figure 3.15. At a higher speed, or at longer time constant, the traverse time of the magnet passing over the stationary guideway coil is much shorter than the time constant of the stationary guideway coil. The current induced in the stationary guideway coil does not have time to change its direction during the time the magnet passes over the coil. Thus, the lift or the guidance forces have only one positive impulse. The drag force still involves two impulses, but the second, associated with a conservative energy component, is positive, as shown in Figures 3.15b and 3.15d. 


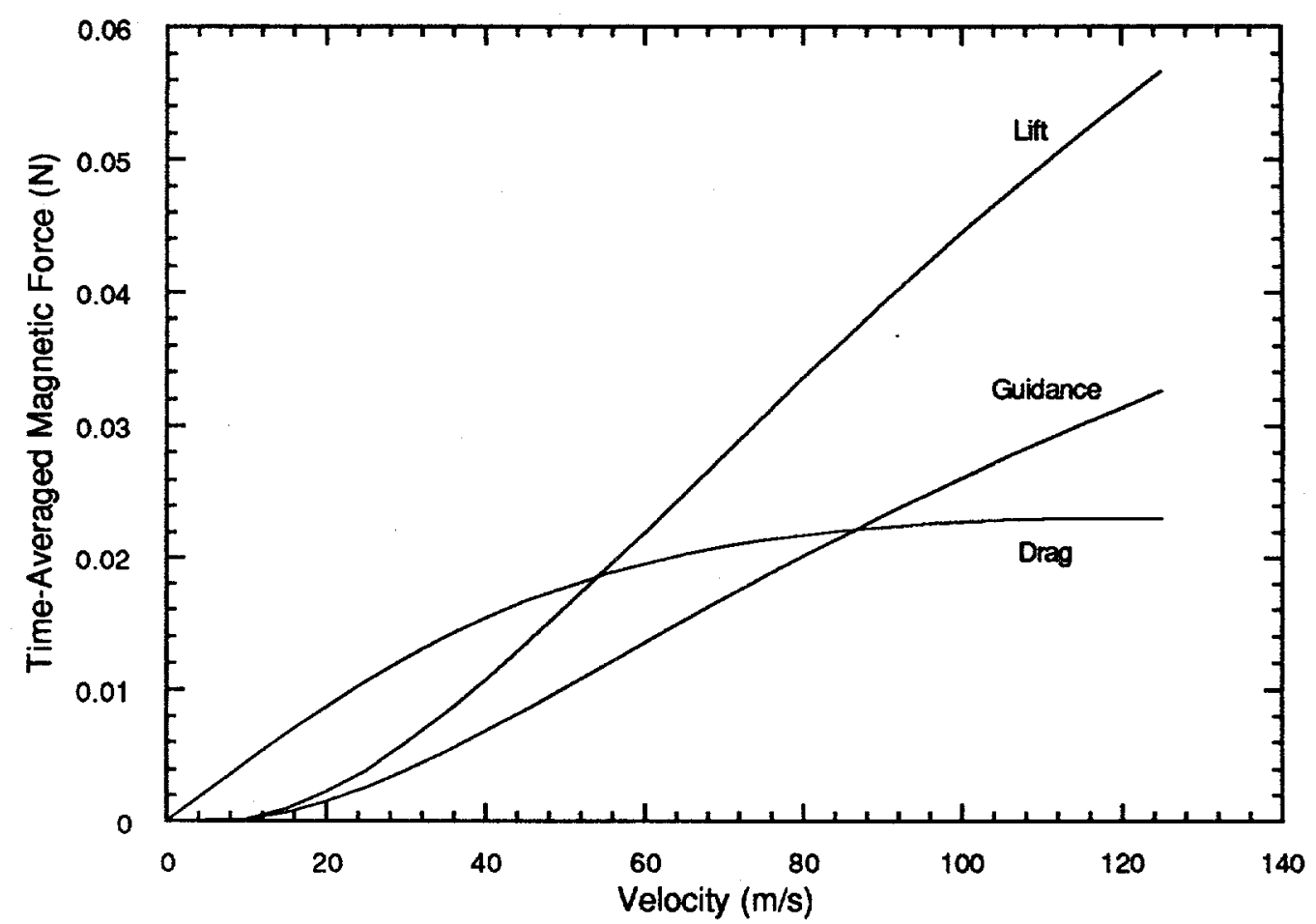

FIGURE 3.10 Time-Averaged Magnetic Forces of Null-Flux Coil Configuration $A$ as a Function of Speed (horizontal displacement $y=5 \mathrm{~mm}$, air gap $z=4 \mathrm{~mm}$ )

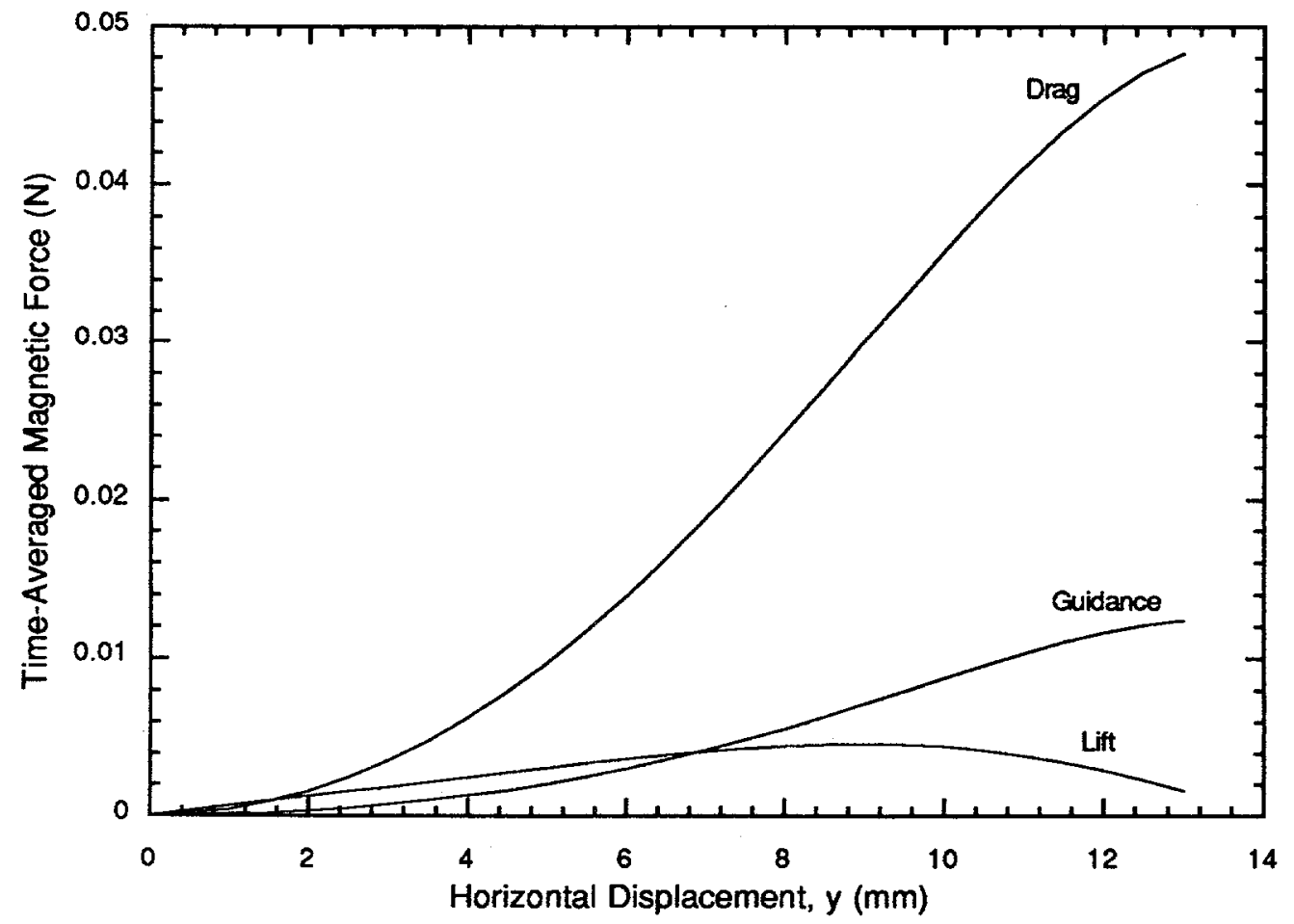

FIGURE 3.11 Time-Averaged Magnetic Forces of Coil Configuration A as a Function of Horizontal Displacement at a Speed of $22.6 \mathrm{~m} / \mathrm{s}$ 


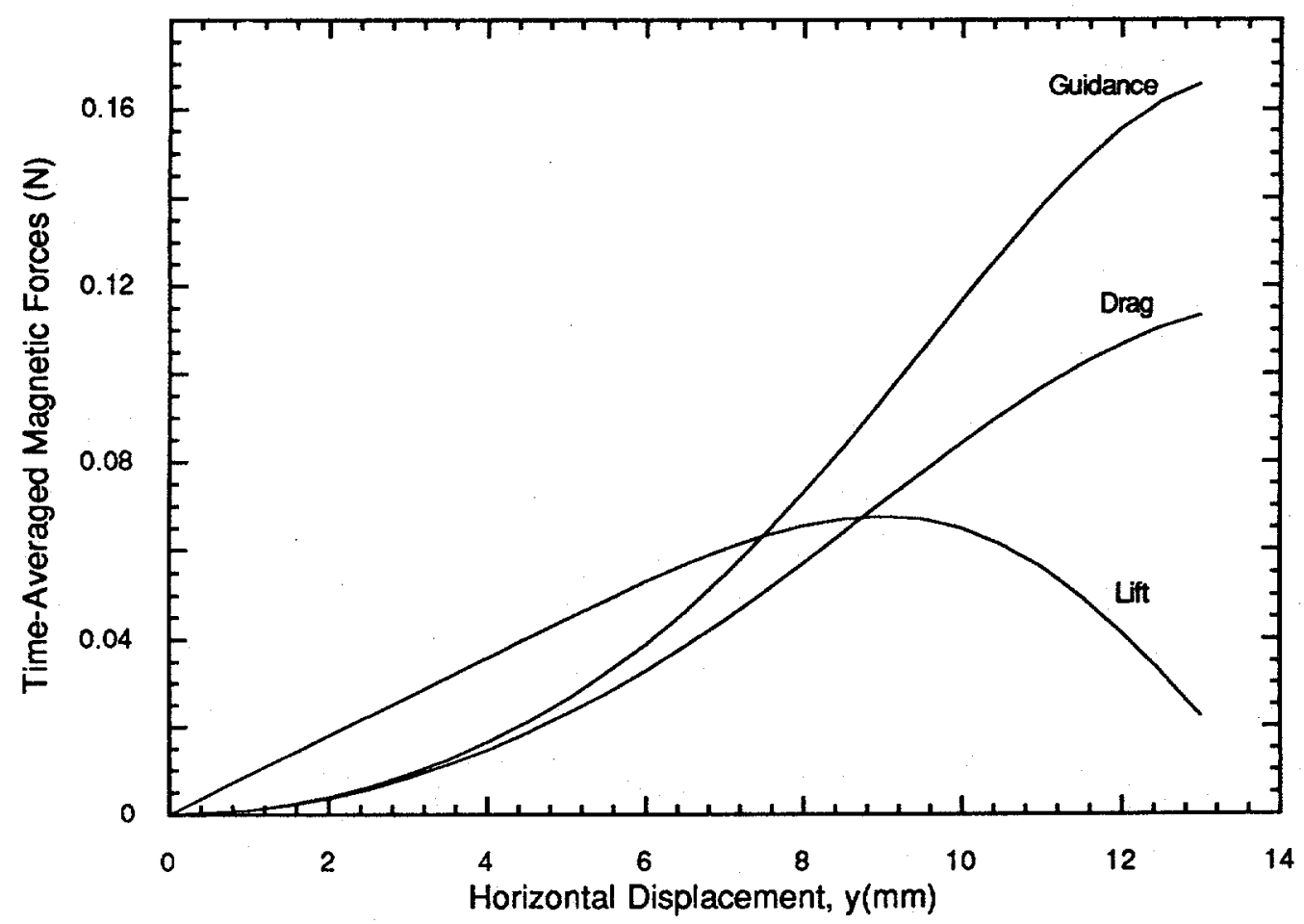

FIGURE 3.12 Time-Averaged Magnetic Forces of Coil Configuration A as a Function of Horizontal Displacement at a Speed of $100 \mathrm{~m} / \mathrm{s}$

\subsection{Dynamic Characteristics of Thick Single-Turn Figure-Eight- Shaped Null-Flux Coil}

For further investigation of the null-flux coil characteristics, a single-turn figure-eightshaped null-flux coil with relatively large conductor cross-section was designed (Figure 3.16). This thick null-flux coil was $50.8-\mathrm{mm}$ high and $25.4-\mathrm{mm}$ wide with a conductor cross-section of $6.4 \times 3.2 \mathrm{~mm}$. Thus, the time constant of the new coil was about 6 times higher than that of the old coils made with No. 12 copper magnet wire.

Results of the computer simulations conducted with the thick single-turn null-flux coil are shown in Figures 3.17 through 3.21. Lift, guidance, and drag forces as a function of time at speeds of $22.6 \mathrm{~m} / \mathrm{s}$ and $100 \mathrm{~m} / \mathrm{s}$ are shown in Figures 3.17 and 3.18 , respectively, where the guidance force ( $\mathrm{z}$ directed) is several times larger than the lift force ( $\mathrm{y}$ directed) because the lateral displacement $(y)$ is large, $11 \mathrm{~mm}$. Note that the first impulse of the lift and guidance force waveforms are substantial. However, the second impulse of both waveforms are much reduced because of the increase of the time constant of the null-flux coils. This leads to a reasonable value of the time-averaged magnetic forces as shown in Figure 3.19, where the lift-to-drag ratio can be calculated to be 13 at a speed of $120 \mathrm{~m} / \mathrm{s}$ for a lateral displacement $\mathrm{y}=5 \mathrm{~mm}$.

Figures 3.20 and 3.21 show the dependence of the time-averaged magnetic forces and the lift-to-drag ratio on the lateral displacement (y) at speeds $22.6 \mathrm{~m} / \mathrm{s}$ and $100 \mathrm{~m} / \mathrm{s}$, respectively. As 


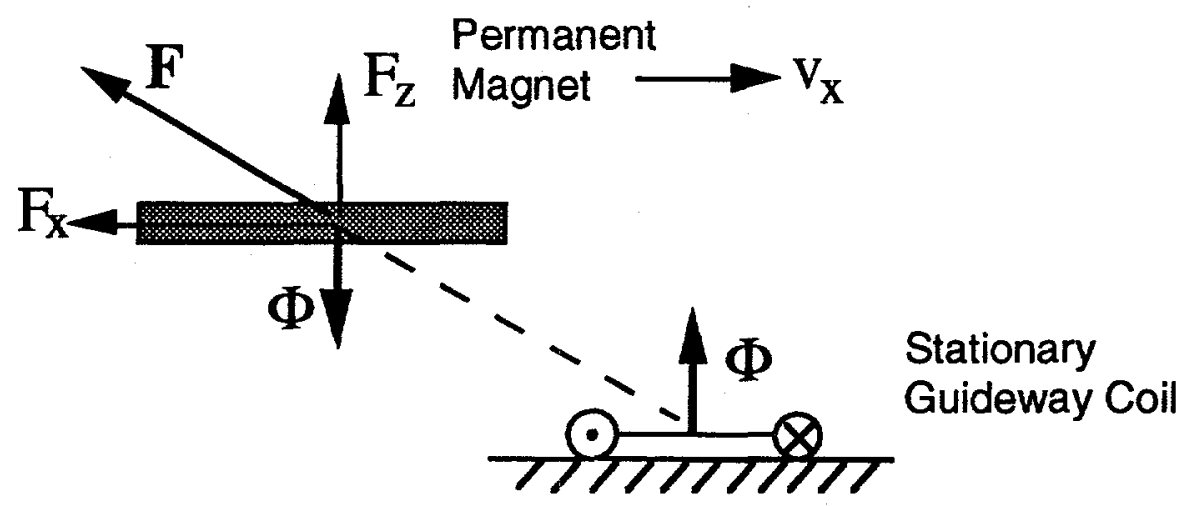

(a) A Permanent Magnet approaching a Stationary Guideway Coil

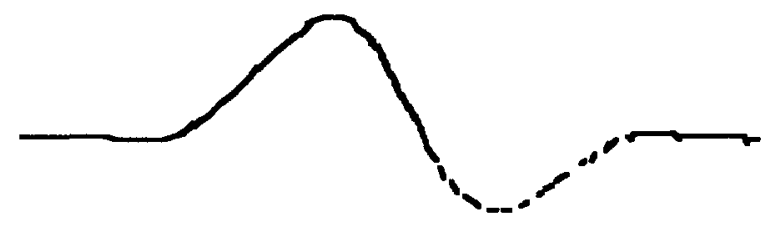

(b) Lift Force Waveform

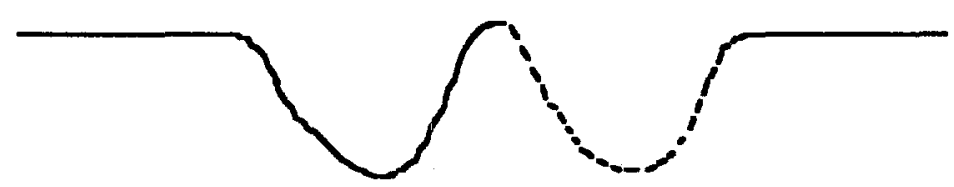

(c) Longitudinal Force Waveform

FIGURE 3.13 Force Characteristics of a Permanent Magnet Approaching a Stationary Guideway Coil 


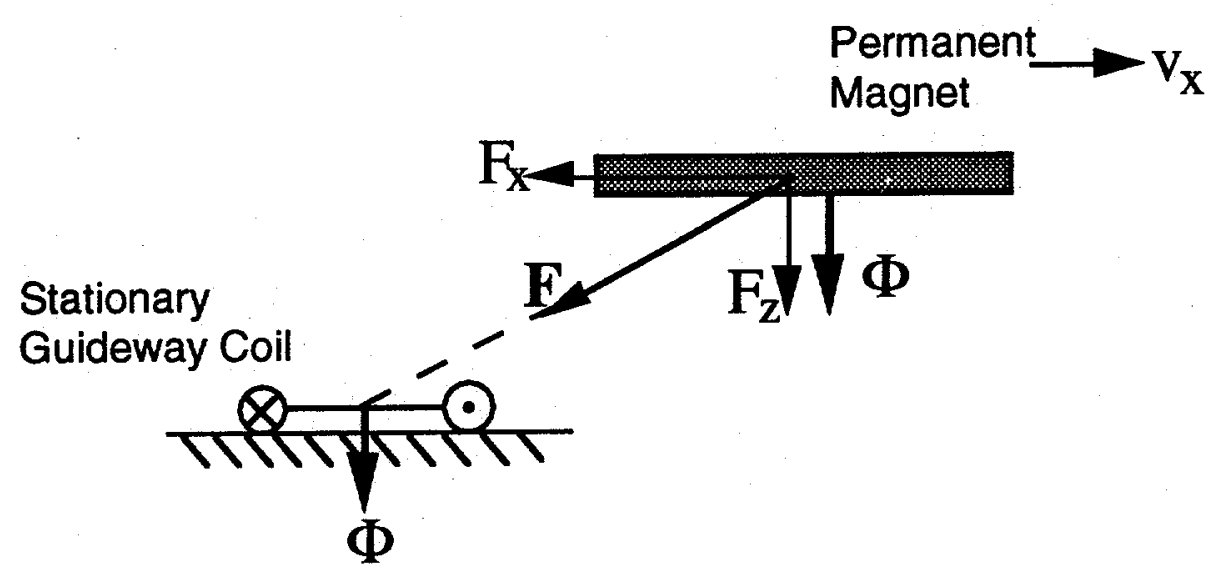

(a) Permanent Magnet Departure from the Stationary Guideway Coil

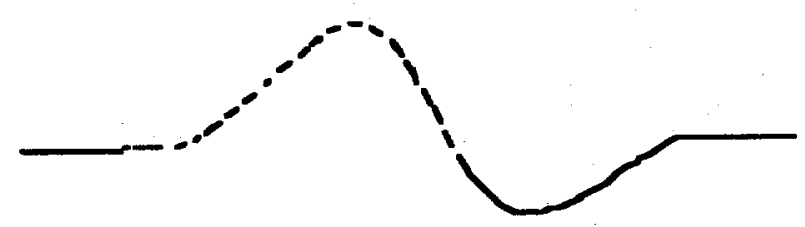

(b) Lift Force Waveform

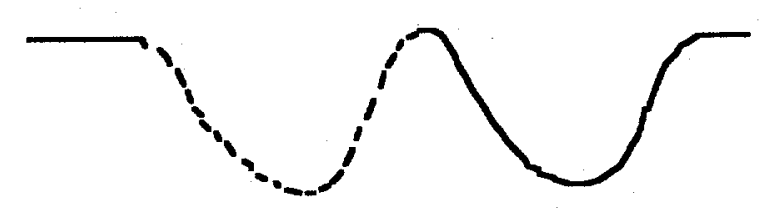

(c) Longitudinal Force Waveform

FIGURE 3.14 Force Characteristics of a Permanent Magnet Departing from a Stationary Guideway Coil 


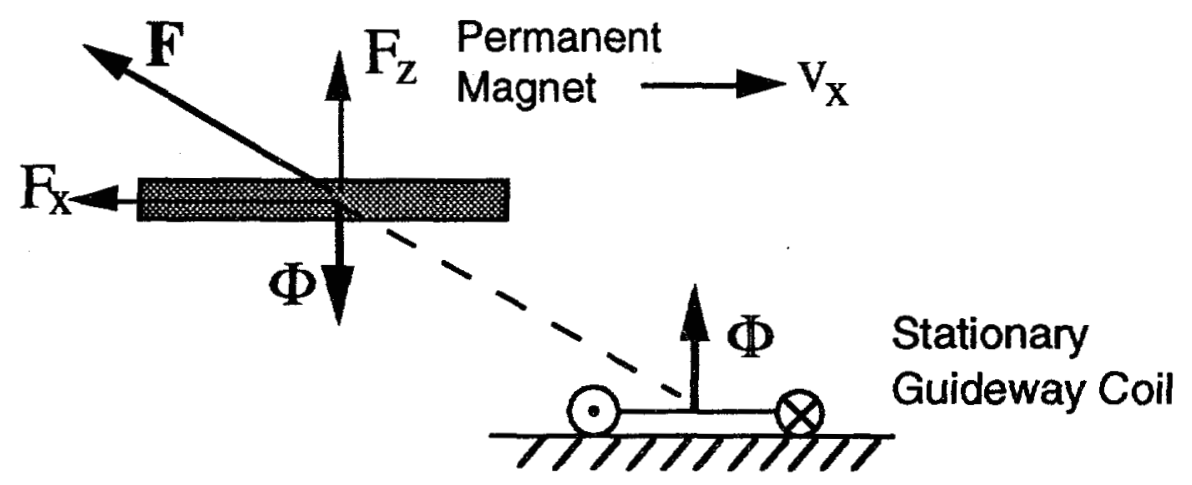

(a) Permanent Magnet Approching a Stationary Guideway Coil at High Speed or High Time Constant

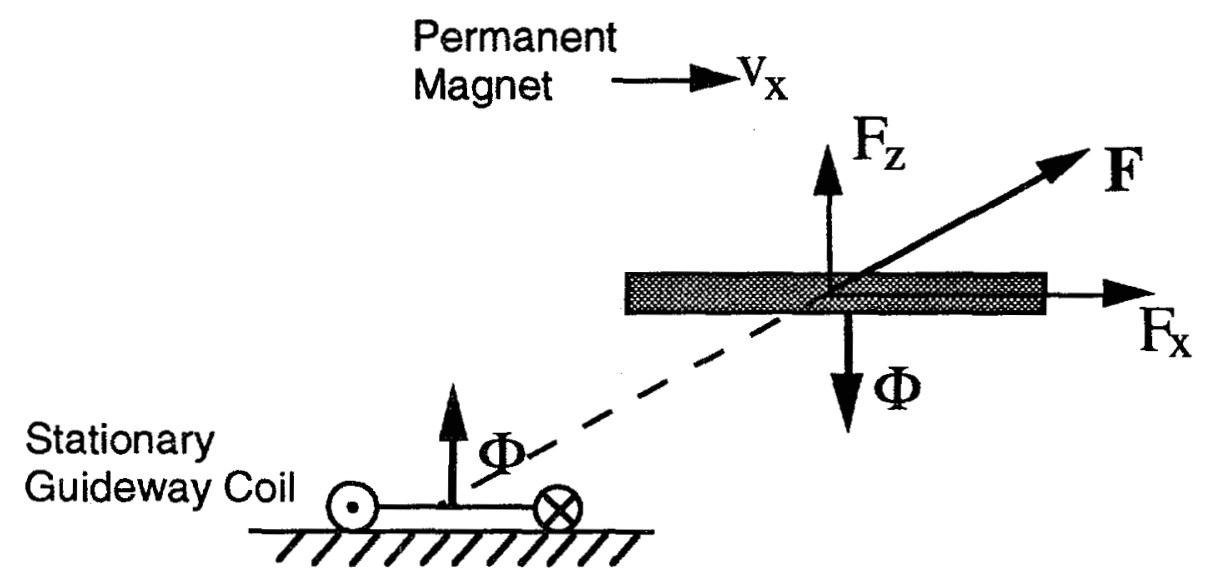

(b) Permanent Magnet Departure from a Stationary Guideway Coil at High Speed or High Time Constant

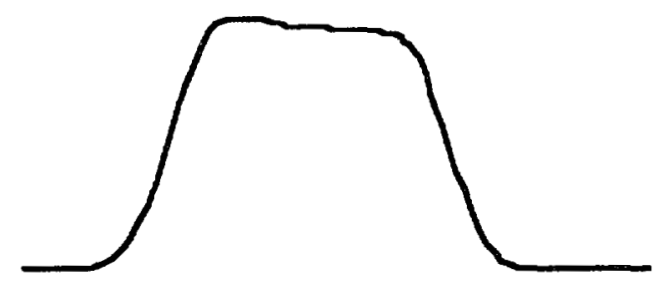

(c) Lift Force Waveform

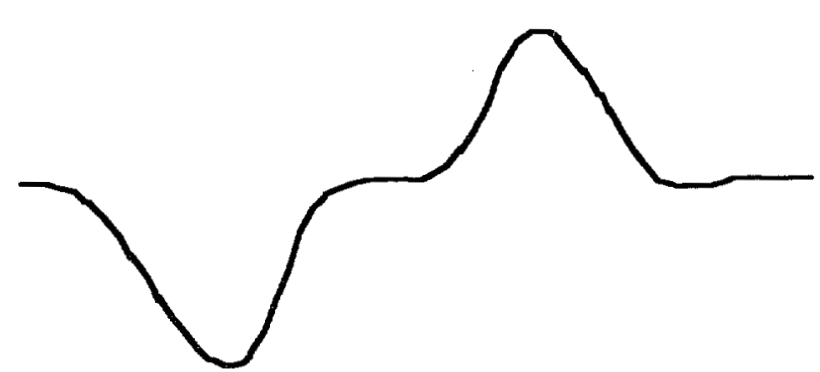

(d) Longitudinal Force Waveform

FIGURE 3.15 Force Characteristics of a Permanent Magnet Passing over a Stationary Guideway Coil at High Speed or High Time Constant 

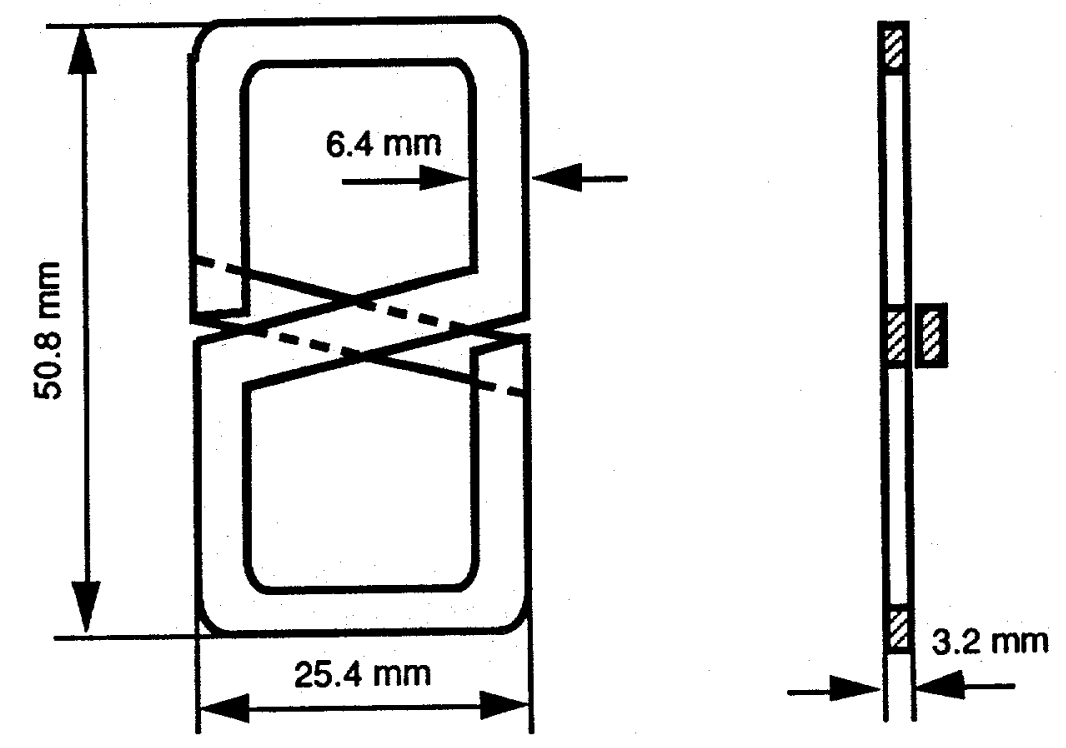

FIGURE 3.16 Thick Single-Turn Figure-Eight-Shaped Null-Flux Coil

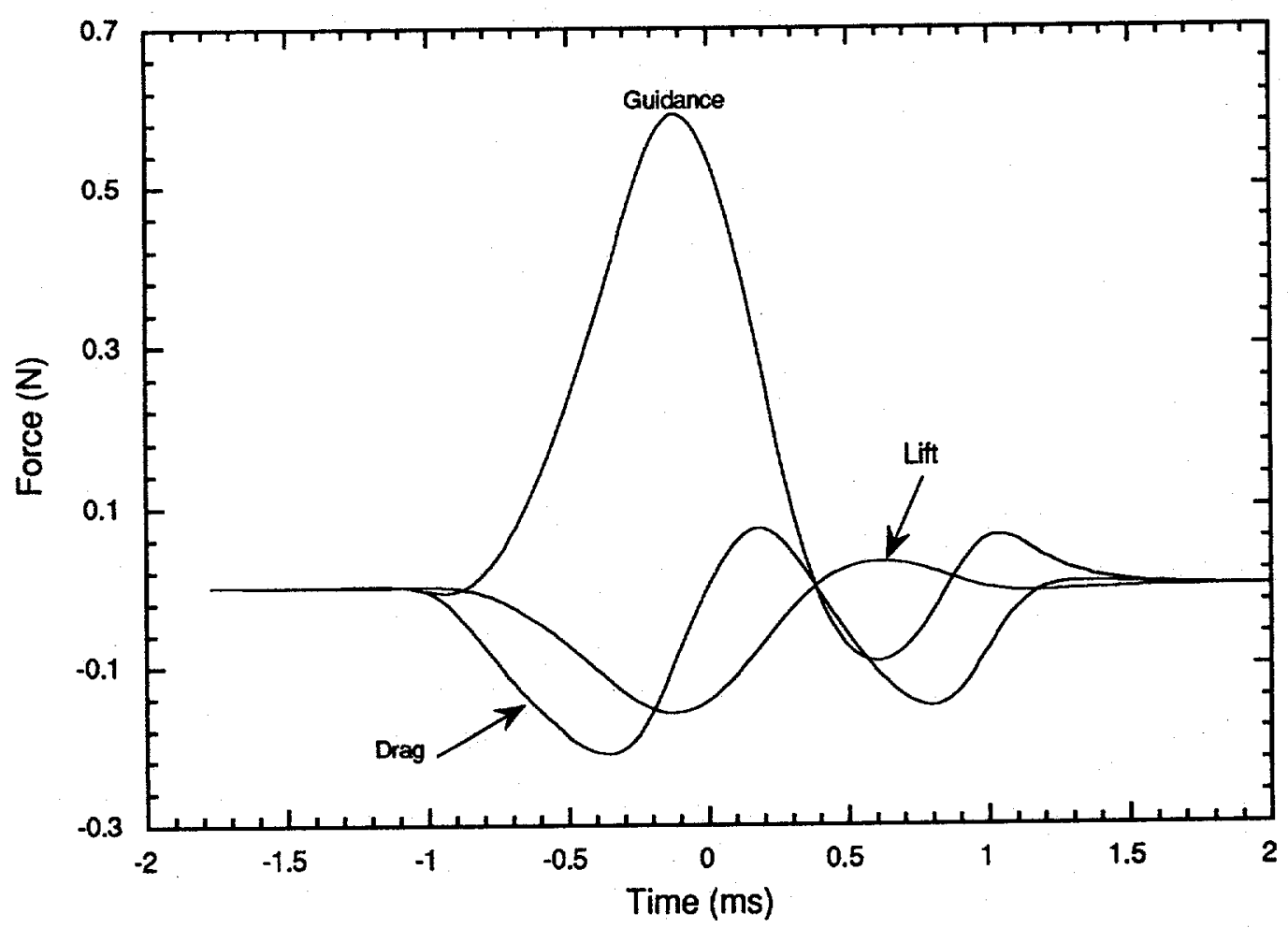

FIGURE 3.17 Magnetic Force Waveforms at a Speed of $22.6 \mathrm{~m} / \mathrm{s}$ and a Lateral Displacement of $11 \mathrm{~mm}$ 


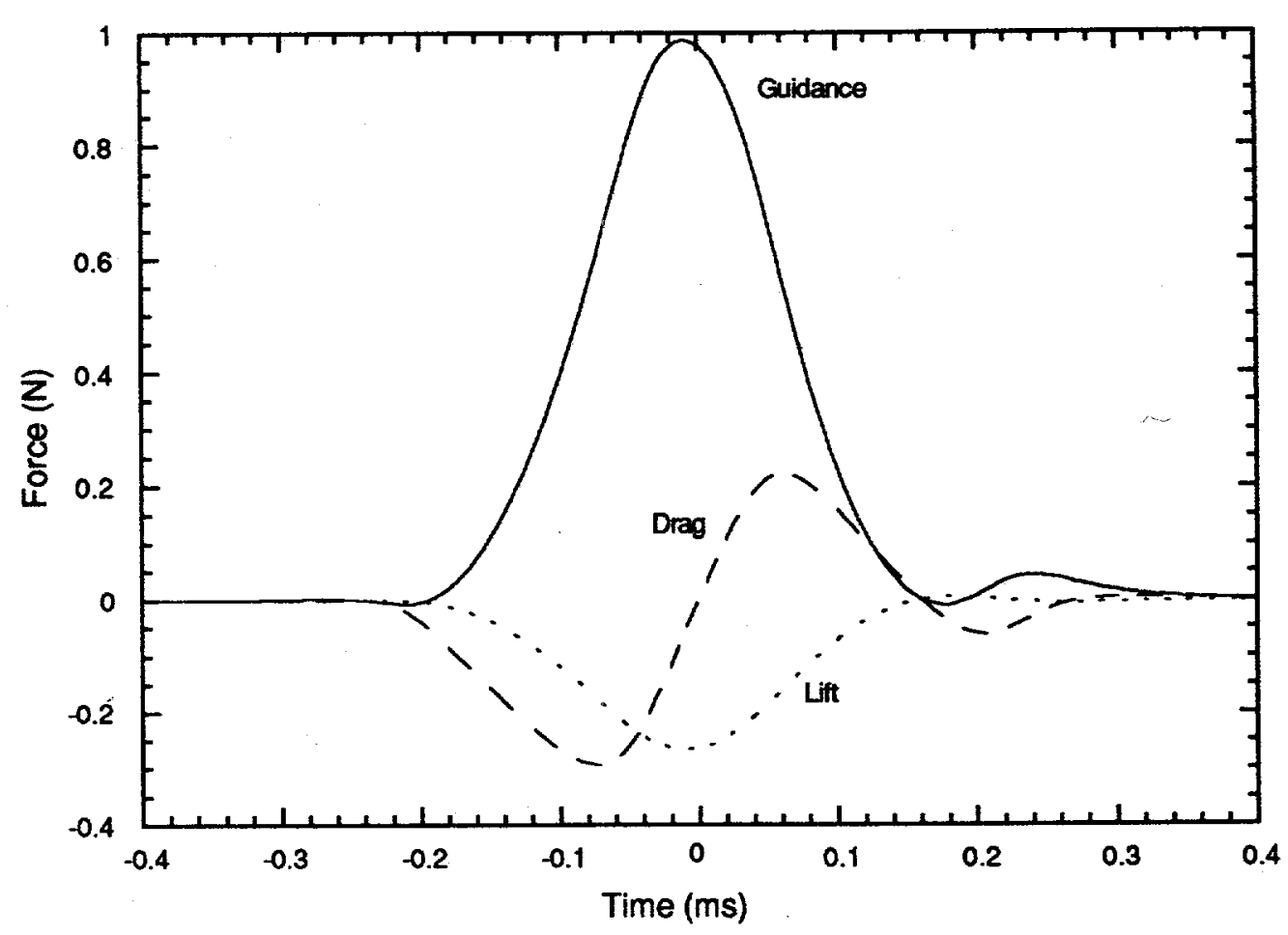

FIGURE 3.18 Magnetic Force Waveforms at a Speed of $100 \mathrm{~m} / \mathrm{s}$ and a Lateral Displacement of $11 \mathrm{~mm}$

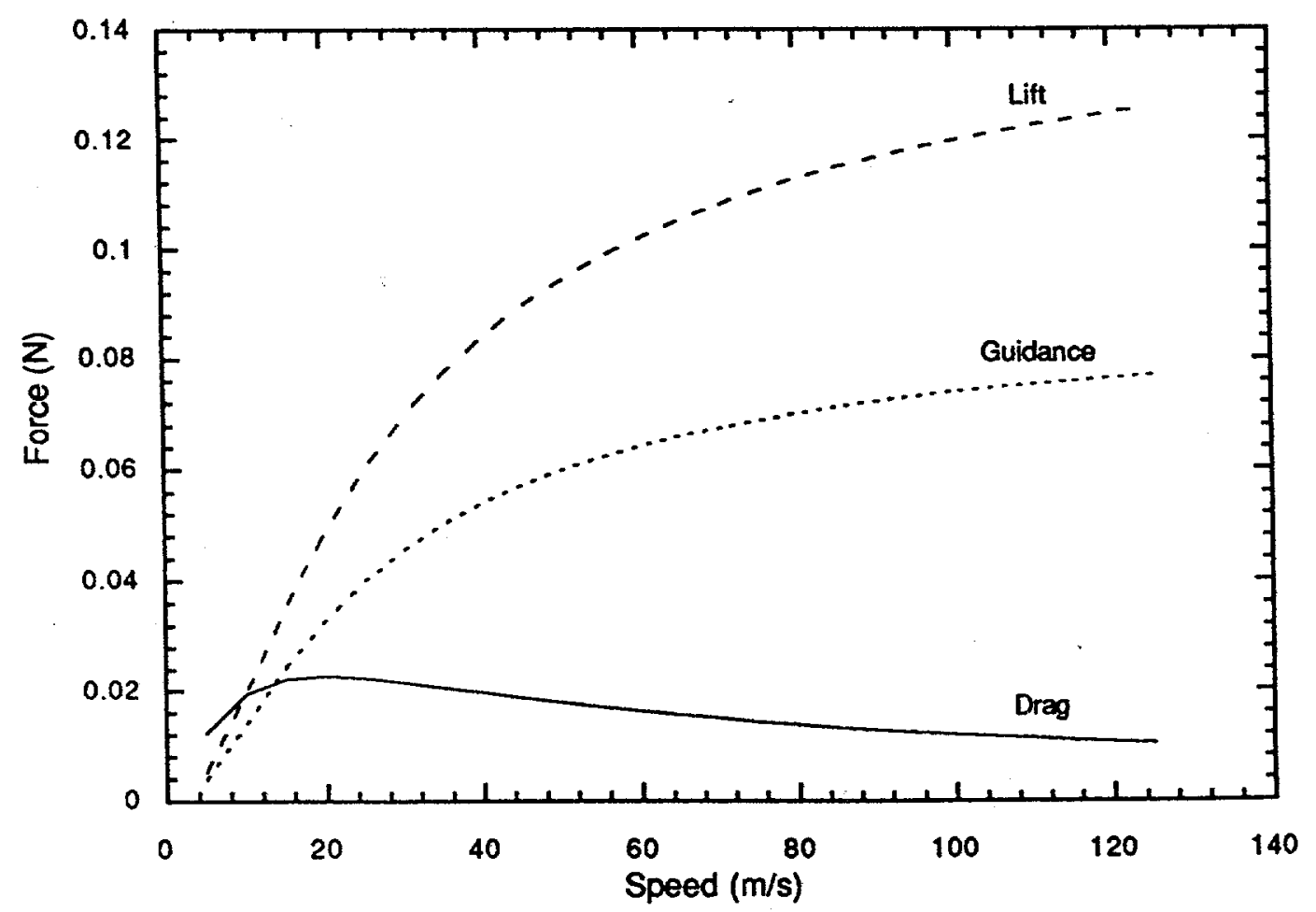

FIGURE 3.19 Dependence of Time-Averaged Magnetic Force on Speed at a Lateral Displacement of $5 \mathrm{~mm}$ 


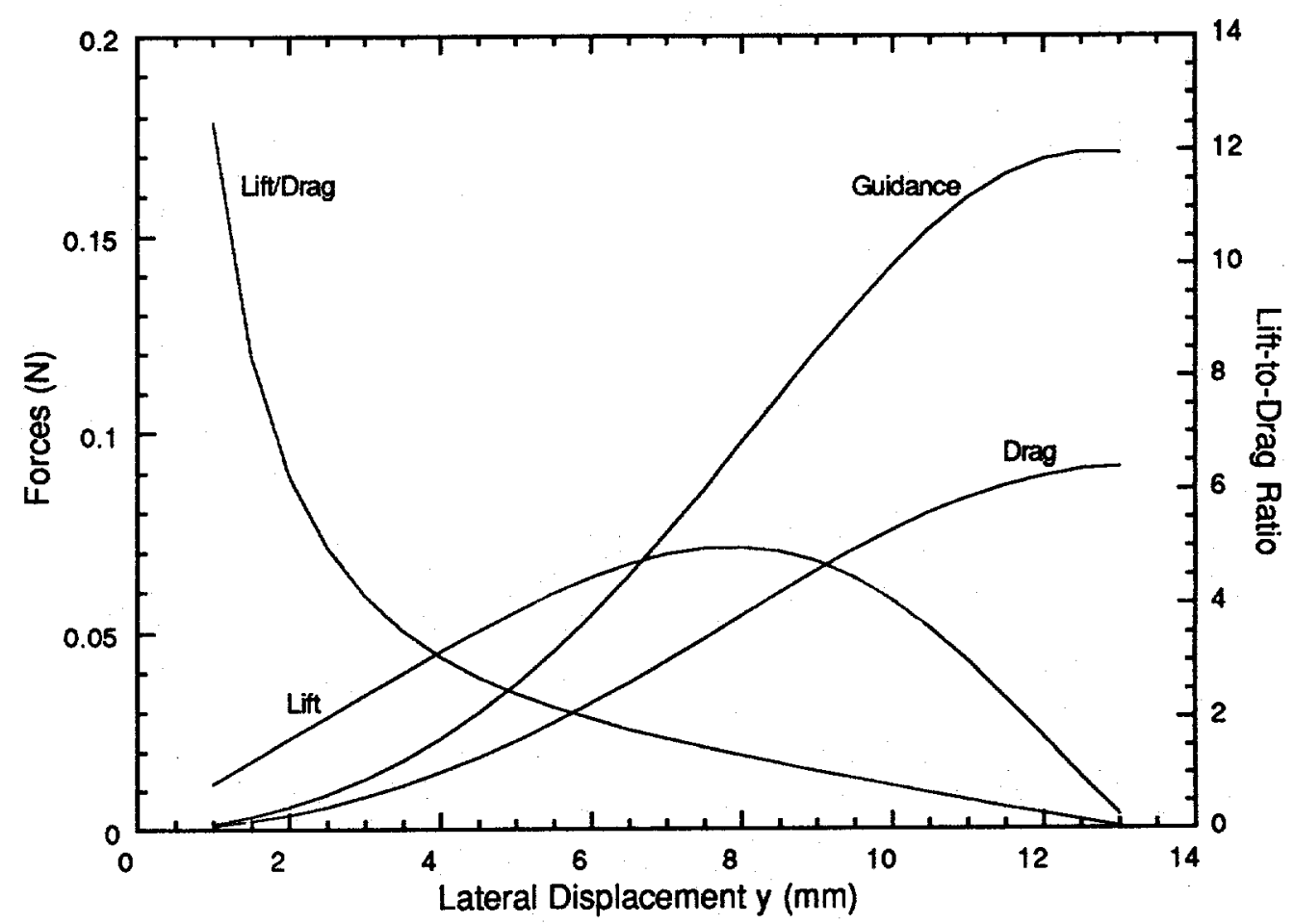

FIGURE 3.20 Time-Averaged Magnetic Forces as a Function of Lateral Displacement at a Speed of $22.6 \mathrm{~m} / \mathrm{s}$

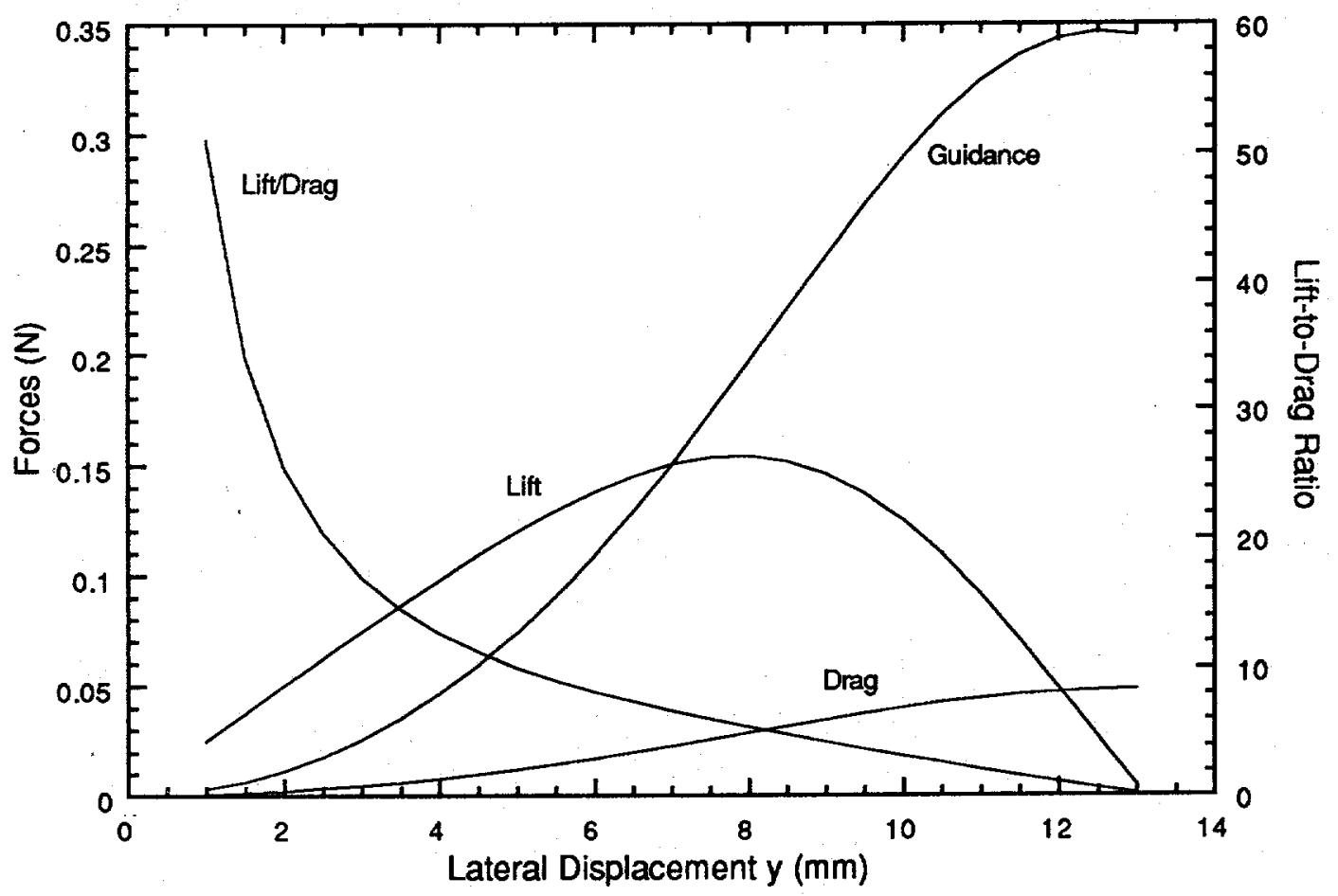

FIGURE 3.21 Time-Averaged Magnetic Forces as a Function of Lateral Displacement at a Speed of $100 \mathrm{~m} / \mathrm{s}$ 
expected, for figure-eight-shaped null-flux coil suspension systems, the lift-to-drag ratio approaches infinity at the lateral displacement null-flux equilibrium position, $y=0$. The lift force increases for a small lateral displacement approaches a maximum value at a lateral displacement of about $8 \mathrm{~mm}$ and then decreases for lateral displacement larger than $8 \mathrm{~mm}$. The guidance and drag forces, however, continue to increase as the lateral displacement increases and reaches a maximum value at a lateral displacement of about $13 \mathrm{~mm}$, where the equivalent SCM is centered with one of the loops of the figure-eight-shaped null-flux coil.

Various experiments were conducted with the thick figure-eight-shaped null-flux coil. Testing indicated that the thick coil increased the measurement range, as expected, by a factor of four. The lift force characteristics displayed the expected dependence upon lateral displacement $(y)$. As shown in Figures 3.20 and 3.21, the lift force maximized at $y= \pm 8 \mathrm{~mm}$ away from the nullflux region, and went to zero at $y= \pm 13 \mathrm{~mm}$. The latter position is indicative of when the magnet passes directly over one of the loops. This position also represents where the drag and guidance forces maximize.

However, as the magnet is approached by the center of the coil, the time histories failed to display the expected null-flux characteristics $\left(F_{x}=F_{y}=F_{z}=0\right)$. While the lift force tended to minimize near the center of the coil, the drag and guidances forces maintained relatively large impulses. This is because of the skin effect resulting from the eddy current loss in the relatively larger cross-section of the conductor. This suggests that the parallelly-connected multi-turn coil is necessary to reduce the skin effect.

\subsection{Multi-Turn Figure-Eight-Shaped Null-Flux Coil Experiment}

In an effort to reduce the skin-effect that occurs in the thick single-turn null-flux coil, several multi-turn figure-eight-shaped null-flux coils were designed and tested. Various wire gauges were tested in an attempt to utilize the full cross-sectional area allowed by the physical constraints of the system. The most efficient coil was a nine-turn, 16 AWG copper coil with a single crossover within the plane of the coil. This coil was tested for various displacements.

The nine-turn series coil displayed the natural null-flux characteristics, absent in the thickcoil trials, thus reinforcing the eddy current explanation. The force signals were well behaved over the entire lateral y-displacement. Each force impulse component displayed the expected dependency on lateral displacement seen in the first two experiments ( single-turn thin and singleturn thick ). The lift force maximized around $y= \pm 8 \mathrm{~mm}$ and went to zero around $\mathrm{y}= \pm 13 \mathrm{~mm}$, while drag and guidance maximized at around $y= \pm 13 \mathrm{~mm}$ position. The one concern about this coil was that it only increased the measurement range by a factor of slightly over two.

Once the experimental data was gathered for the various coils, it was necessary to determine the flux density of the permanent magnet. Since the COILGDWY code models a SCM moving past null-flux coils, it is necessary to model the permanent magnet as a superconducting 
coil with a certain number of ampere turns $(A-T)$, or superconducting current $I_{S}$. The force on the magnet is directly proportional to the current $I_{S}$, so this term must be known with some accuracy.

The magnetic flux of the magnet was measured using a standard Hall probe and Labview data acquisition application. The field was measured along the vertical and horizontal sides, as well as through the center of the magnet. The data was then compared to computationally produced data for a SCM of the same dimensions and various amounts of current. The results are shown in Figures 3.22 and 3.23. Figure 3.22 shows the simulated flux density curve using a current value of 1930 A-T. Figure 3.23 shows the experimental flux density using the Hall probe data. The slight magnetic anomaly on the right peak of the experimental data curve, Figure 3.23, is most likely due to demagnetization from the longstanding relative motion of the coils.

The comparisons between computational and experimental data for two multi-turn coils is shown in Figures 3.24 through 3.29. Figures 3.24, 3.25, and 3.26 shows corresponding lift, guidance, and drag comparisons for the nine-turn series coil. Overall, the results for this coil were excellent, with only around a 5\% difference between the computer-based results and the experimental results.

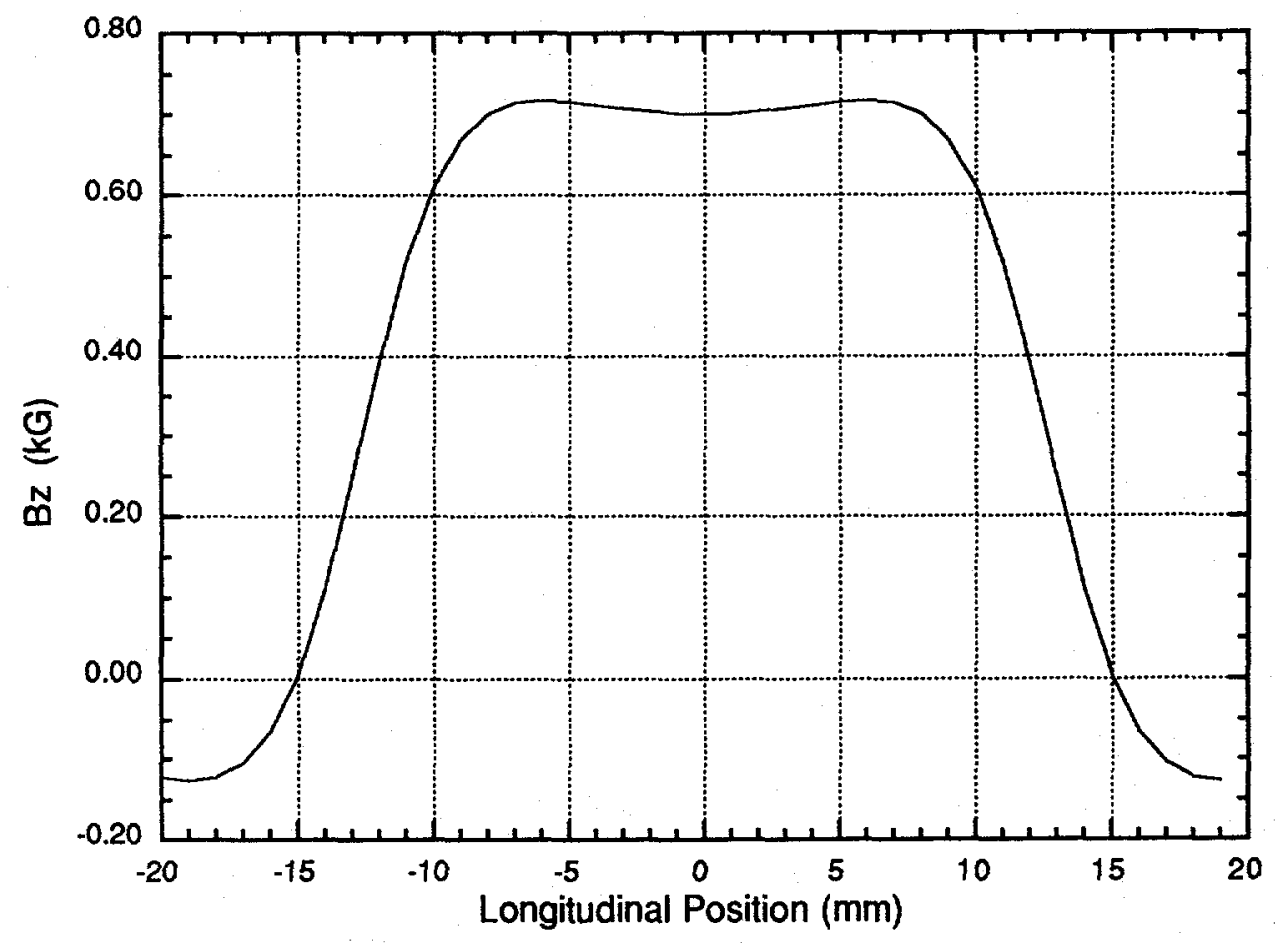

FIGURE 3.22 Calculated Flux Density Produced by a Superconducting Coil as a Function of Lateral Distance (SCM coil with $1930 \mathrm{~A}-\mathrm{T}$, simulating a permanent magnet $25.4 \mathrm{~mm}$ square by $3.2 \mathrm{~mm}$ thick) 


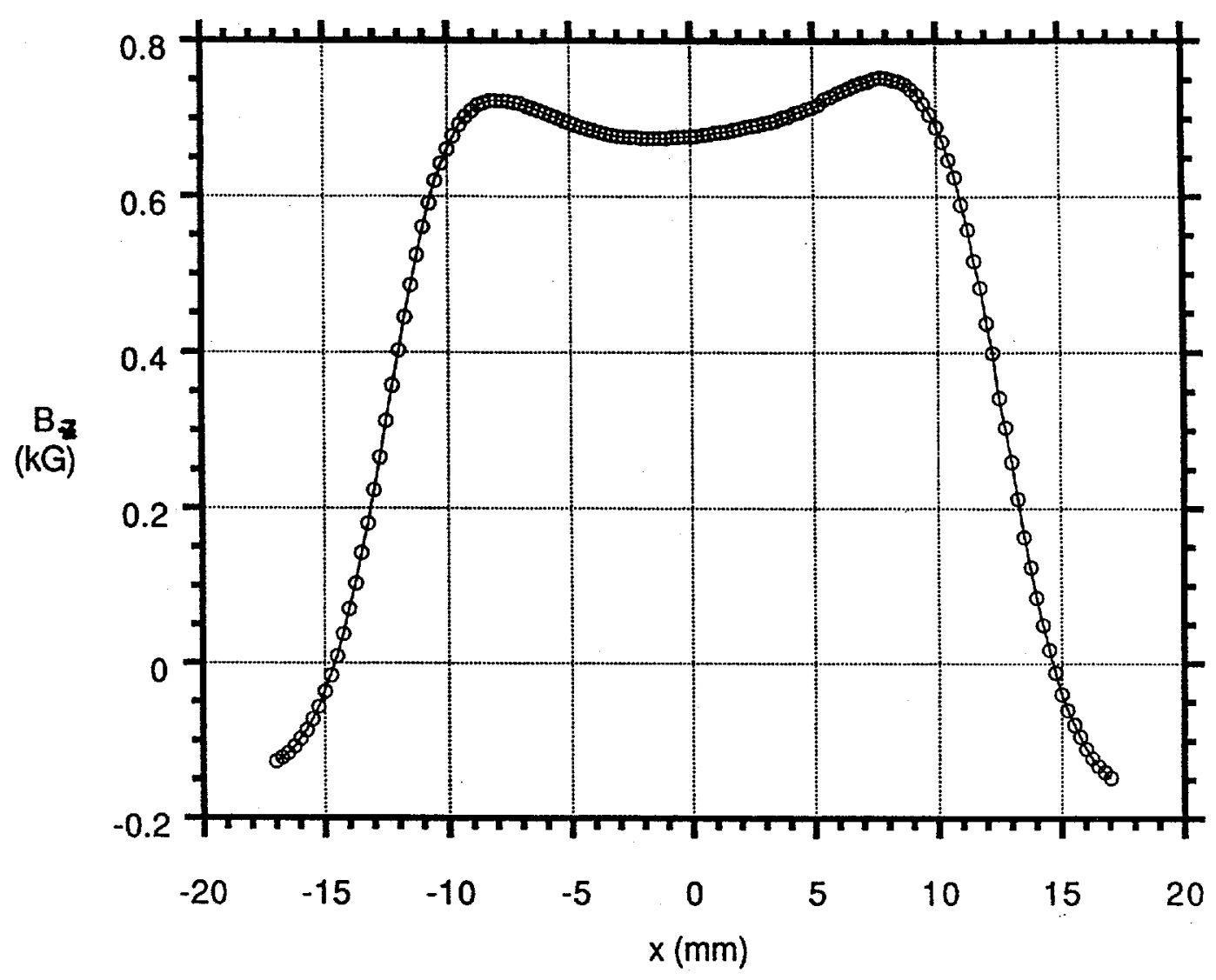

FIGURE 3.23 Flux Density Measured on Permanent Magnet using Standard Hall Probe (magnet is $25.4 \mathrm{~mm}$ square by $3.2 \mathrm{~mm}$ thick)

Figures $3.27,3.28$, and 3.29 show the sample data comparisons for two five-turn parallel coils. Although the theoretical data maintains the same shape as the experimental data, there is a difference in magnitude between 20-25\%, with the experimental being the larger of the two. Possible explanations for this discrepancy include the sensitivity of the system to slight changes in coil orientation, lack of coil symmetry due to method of creation, and difficulty in establishing the coil origin $(y=0)$. 


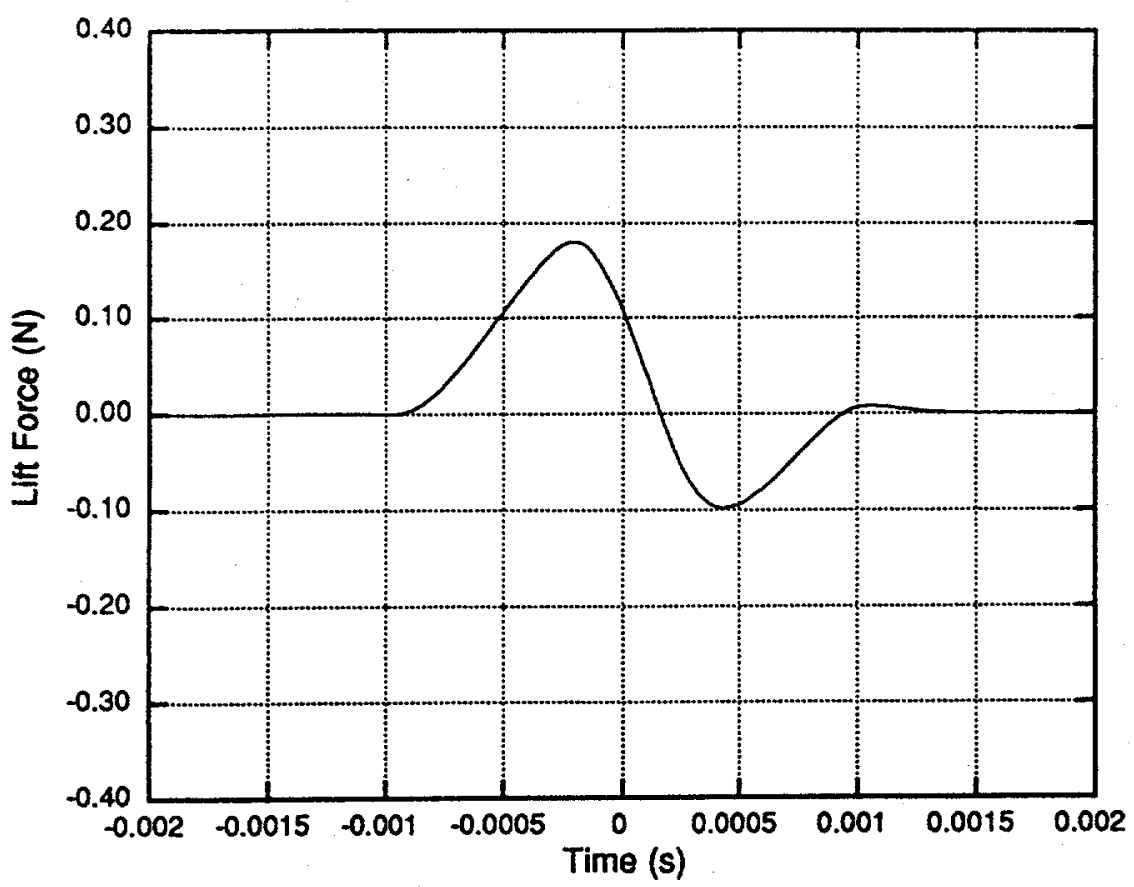

FIGURE 3.24a Computer-Calculated Lift Force Impulse as a Function of Time for Nine-Turn Series Coil $(25.4-\mathrm{mm} \times$ $66.7-\mathrm{mm} \times 4.3-\mathrm{mm}$ null-flux coil incident upon a $25.4-\mathrm{mm}$ by $25.4-\mathrm{mm}$ square, $3.2-\mathrm{mm}$-thick SCM coil at $y=-10.2 \mathrm{~mm}, z=5.65 \mathrm{~mm}$, and speed $=22.6 \mathrm{~m} / \mathrm{s}$ )

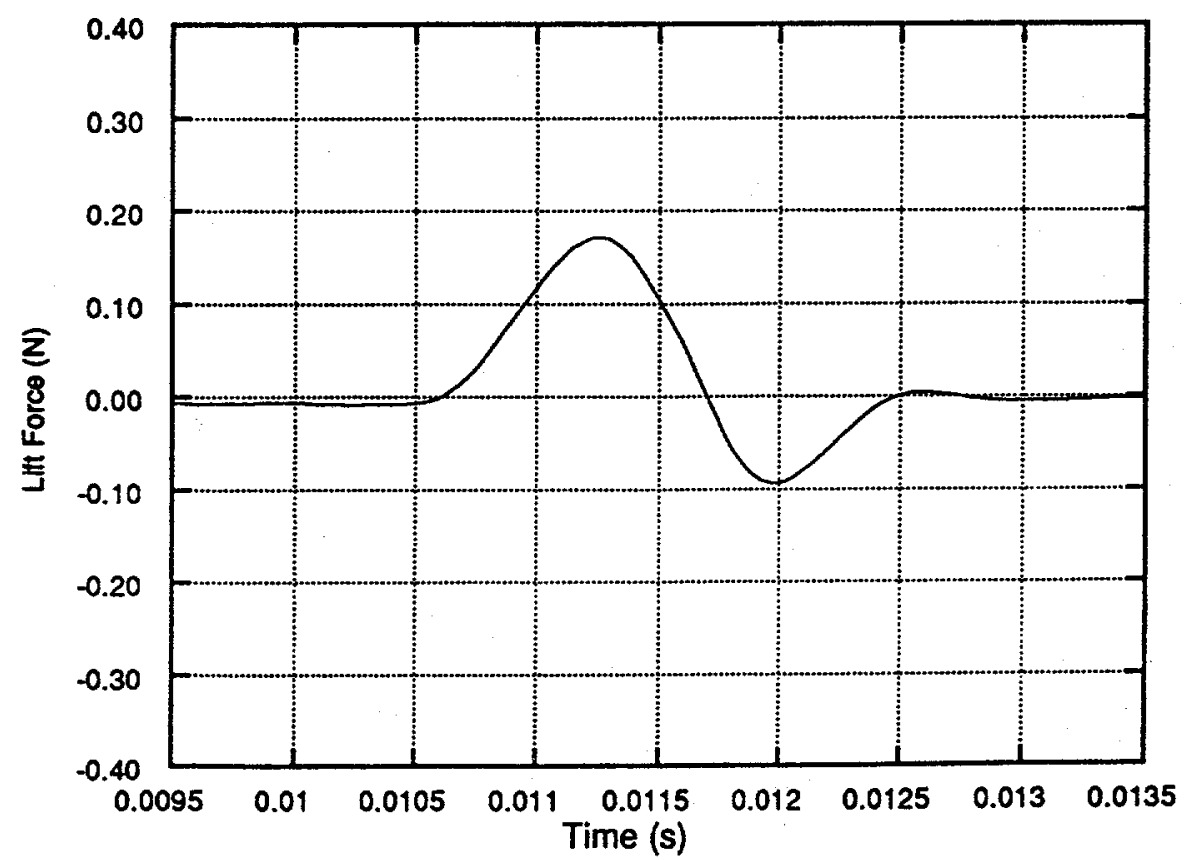

FIGURE 3.24b Experimental Lift Force Impulse as a Function of Time for Nine-Turn Series Coil $(25.4-\mathrm{mm} \times 66.7-\mathrm{mm} \times$ 4.3-mm null-flux coil incident upon a 25.4- $\mathrm{mm}$ square, 3.2-mm-thick $\mathrm{NdFeB}$ permanent magnet at $\mathrm{y}=-10.2 \mathrm{~mm}$, $z=5.65 \mathrm{~mm}$, and speed $=22.6 \mathrm{~m} / \mathrm{s}$ ) 


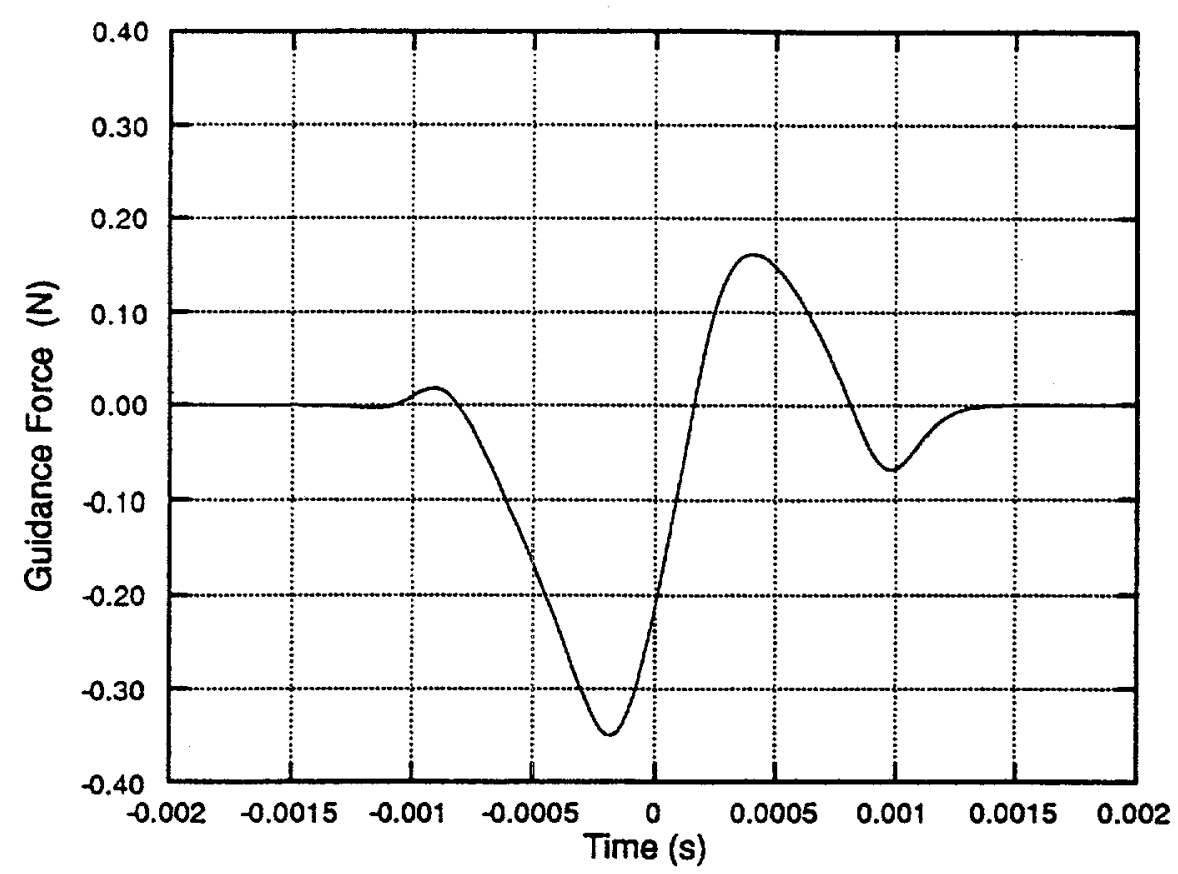

FIGURE 3.25a Computer-Calculated Guidance Force Impulse as a Function of Time for Nine-Turn Series Coil $(25.4-\mathrm{mm} \times$ $66.7-\mathrm{mm} \times 4.3-\mathrm{mm}$ null-flux coil incident upon a $25.4-\mathrm{mm}$ square, 3.2-mm-thick SCM coil at $y=-10.2 \mathrm{~mm}$, $z=5.65 \mathrm{~mm}$, and speed $=22.6 \mathrm{~m} / \mathrm{s}$ )

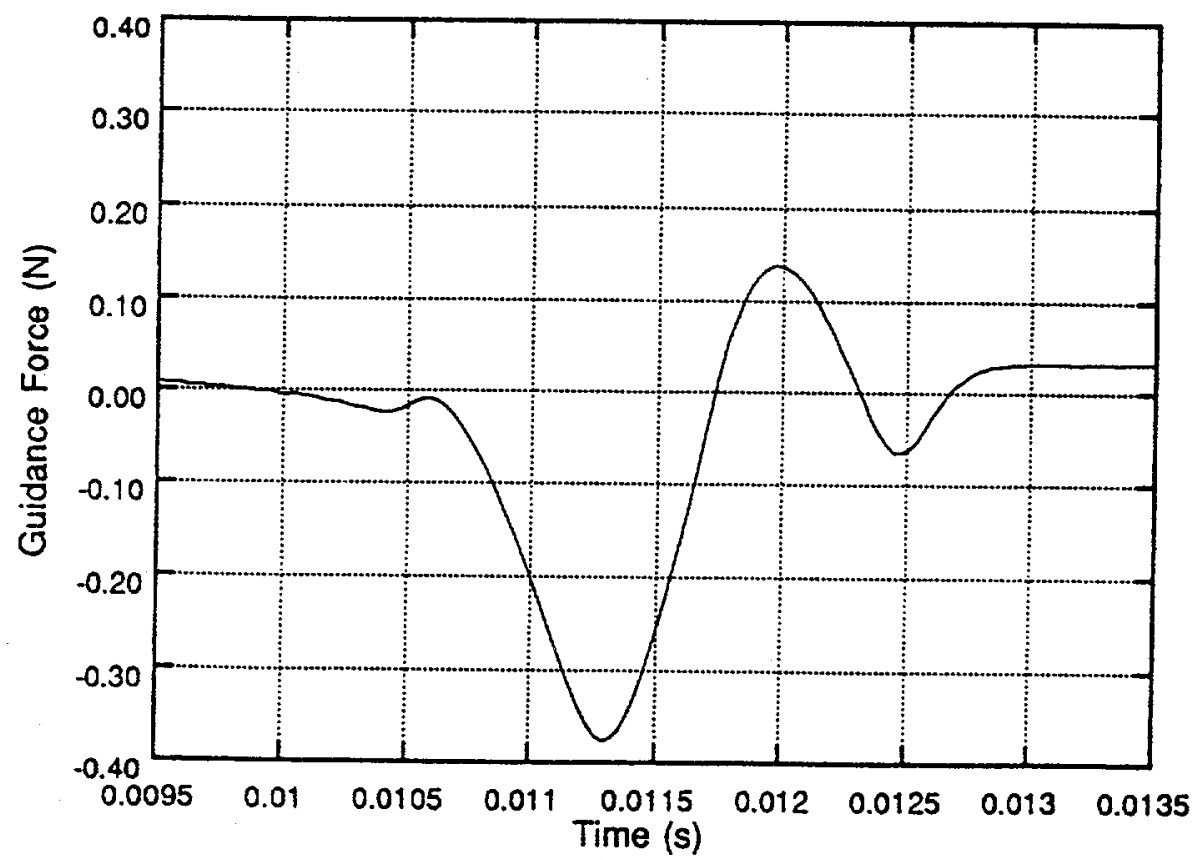

FIGURE 3.25b Experimental Guidance Force Impulse as a Function of Time for Nine-Turn Series Coil (25.4-mm $\times$ $66.7-\mathrm{mm} \times 4.3-\mathrm{mm}$ null-flux coil incident upon a $25.4-\mathrm{mm}$ square, 3.2-mm-thick NdFeB permanent magnet at $y=-10.2 \mathrm{~mm}, z=5.65 \mathrm{~mm}$, and speed $=22.6 \mathrm{~m} / \mathrm{s}$ ) 


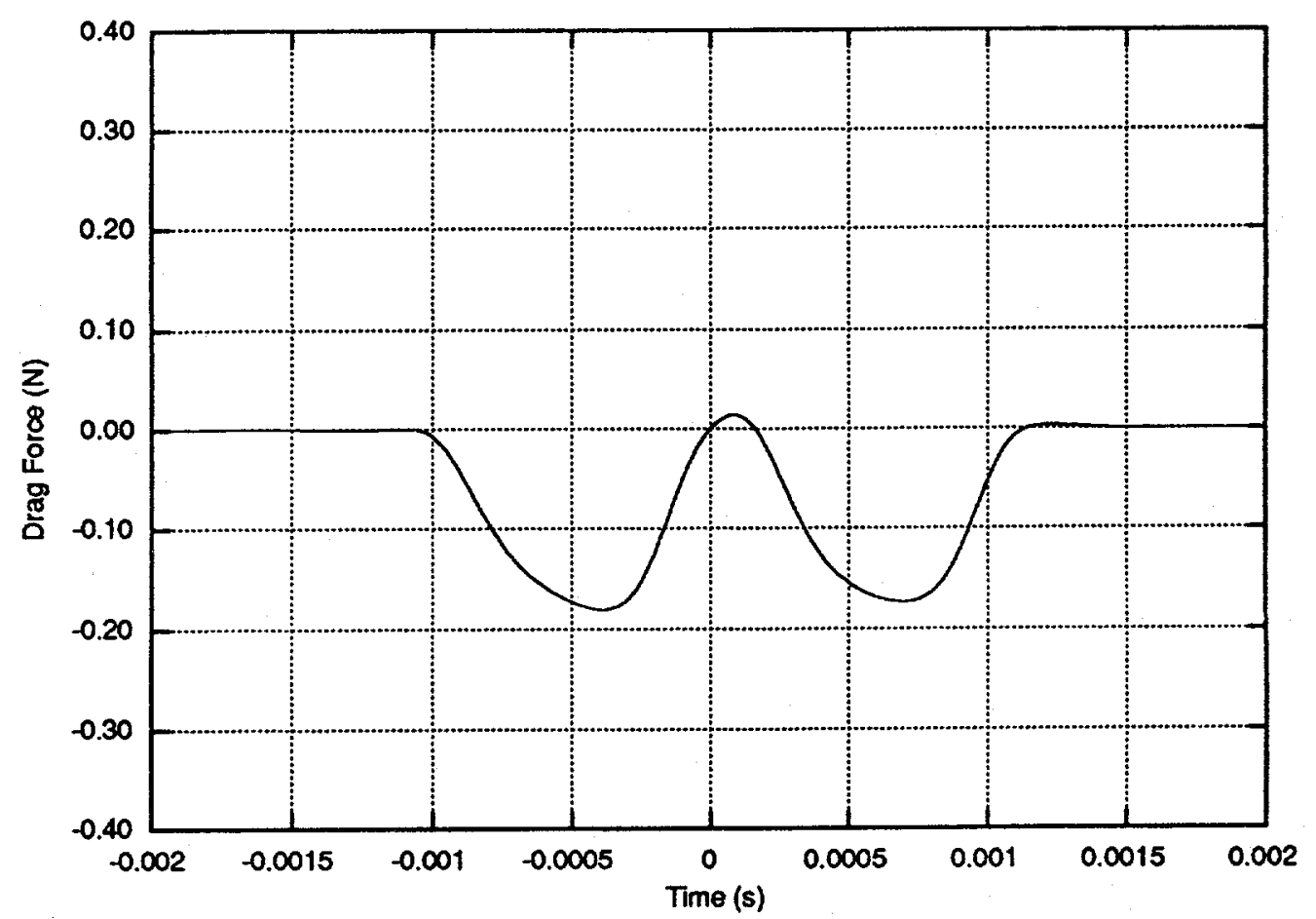

FIGURE 3.26a Computer-Calculated Drag Force Impulse as a Function of Time for Nine-Turn Series Coil $(25.4-\mathrm{mm} \times 66.7-\mathrm{mm} \times 4.3-\mathrm{mm}$ nullflux coil incident upon a $25.4-\mathrm{mm}$ square, 3.2- $\mathrm{mm}$-thick SCM coil at $y=-10.2 \mathrm{~mm}, z=5.65 \mathrm{~mm}$, and speed $22.6=\mathrm{m} / \mathrm{s}$ )

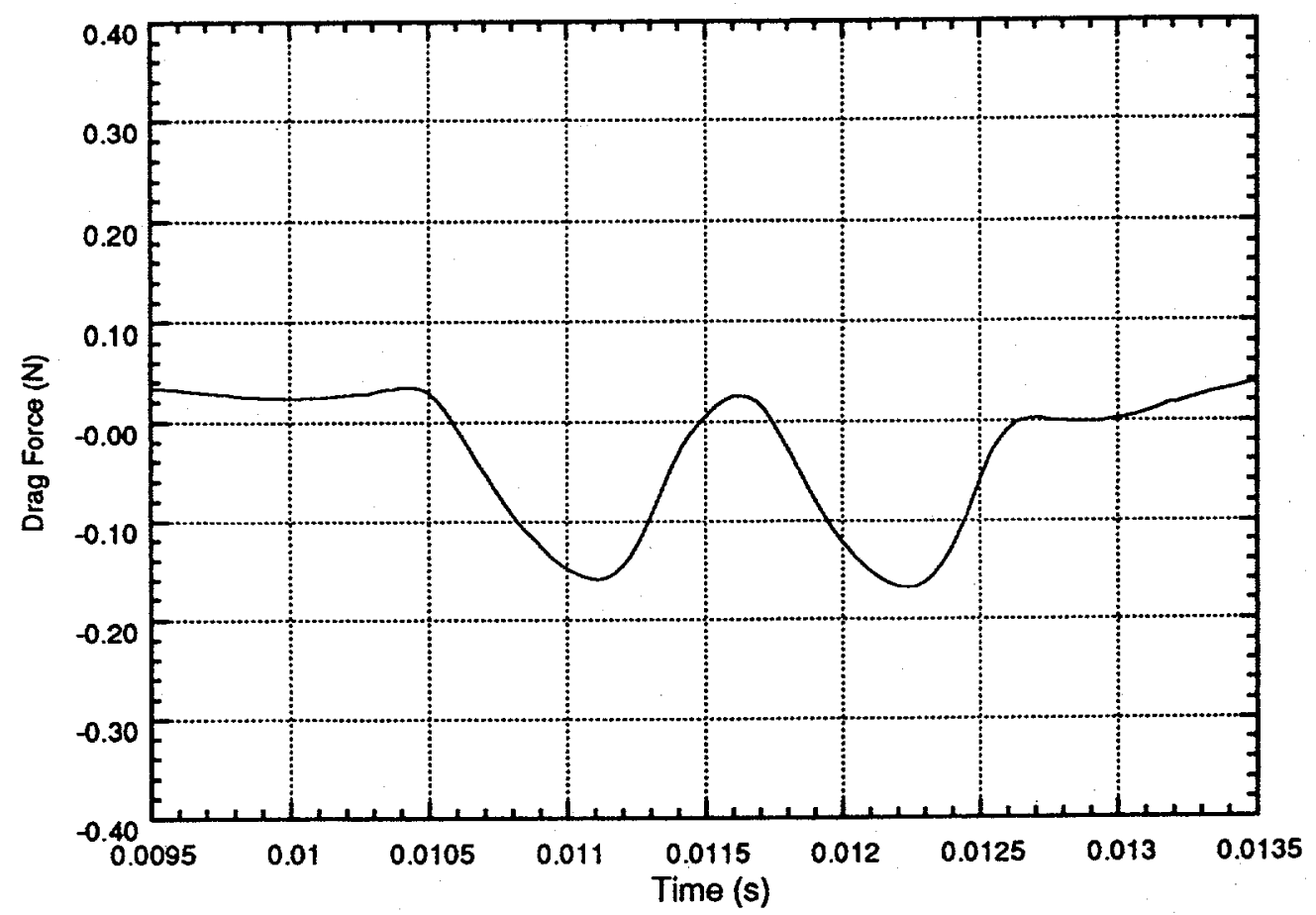

FIGURE 3.26b Experimental Drag Force Impulse as a Function of Time for Nine-Turn Series Coil $(25.4-\mathrm{mm} \times 66.7-\mathrm{mm} \times 4.3-\mathrm{mm}$ null-flux coil incident upon a $25.4-\mathrm{mm}$ square $3.2-\mathrm{mm}$-thick NdFeB Permanent magnet at $y=-10.2 \mathrm{~mm}, z=5.65 \mathrm{~mm}$, and speed $=22.6 \mathrm{~m} / \mathrm{s}$ ) 


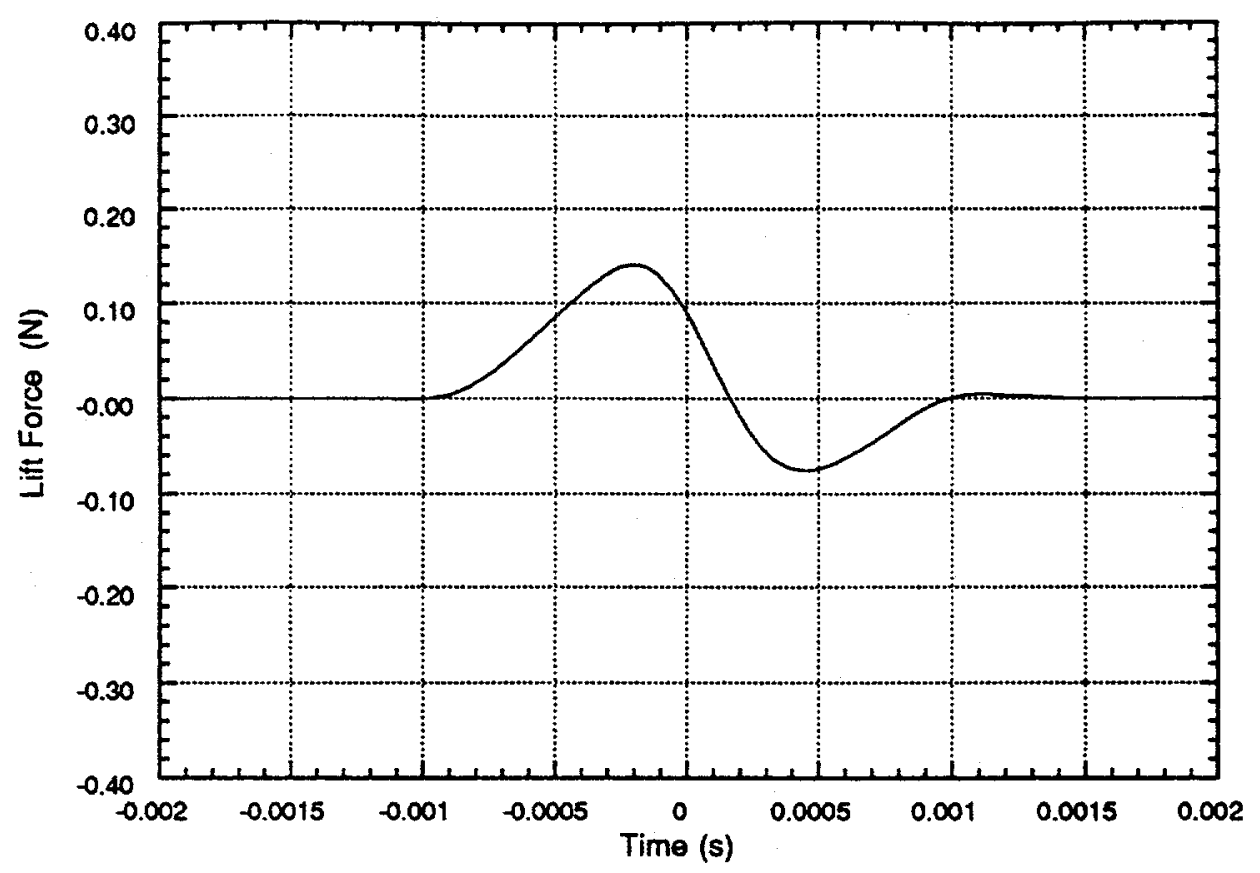

FIGURE 3.27a Computer-Calculated Lift Force Impulse as a Function of Time for Two Five-Turn Parallel Coils $(26.4-\mathrm{mm} \times 62.0-\mathrm{mm} \times$ $5.8-\mathrm{mm}$ null-flux coil incident upon a $25.4-\mathrm{mm}$ square, $3.2-\mathrm{mm}$ thick permanent magnet at $y=-10.2 \mathrm{~mm}, z=6.67 \mathrm{~mm}$, and speed $=22.6 \mathrm{~m} / \mathrm{s}$ )

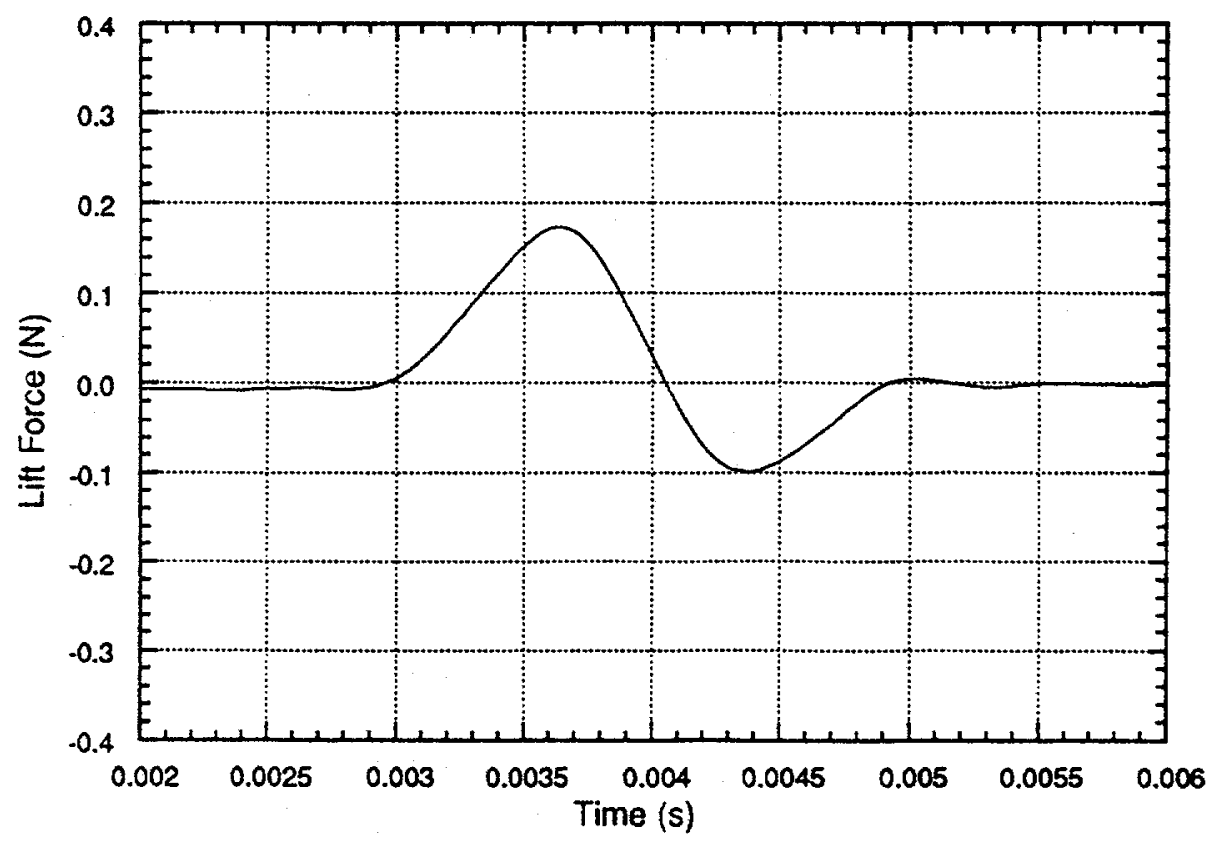

FIGURE 3.27b Experimental Lift Force Impulse as a Function of Time for Two Five-Turn Parallel Coils $(26.4-\mathrm{mm} \times 62.0-\mathrm{mm} \times$ $5.8-\mathrm{mm}$ null-flux coil incident upon a $25.4-\mathrm{mm}$ square, $3.2-\mathrm{mm}$ thick NdFeB permanent magnet at $y=-10.2 \mathrm{~mm}, z=6.67 \mathrm{~mm}$, and speed $=22.6 \mathrm{~m} / \mathrm{s}$ ) 


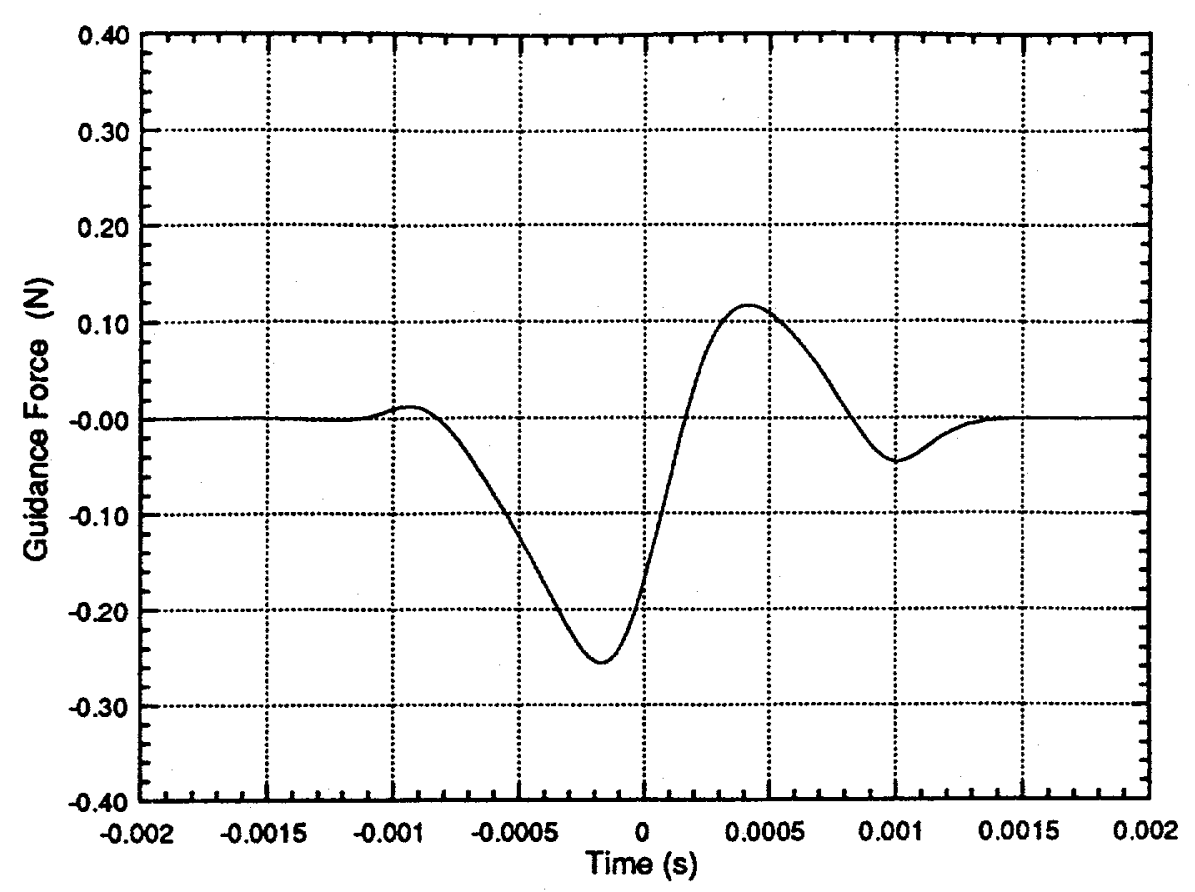

FIGURE 3.28a Computer-Calculated Guidance Force Impulse as a Function of Time for Two Five-Turn Parallel Coils $(26.4-\mathrm{mm} \times$ $62.0-\mathrm{mm} \times 5.8-\mathrm{mm}$ null flux coil incident upon a $25.4-\mathrm{mm}$ square, 3.2-mm-thick permanent magnet at $y=-10.3-\mathrm{mm}$, $z=6.67-\mathrm{mm}$, and speed $=22.6 \mathrm{~m} / \mathrm{s}$ )

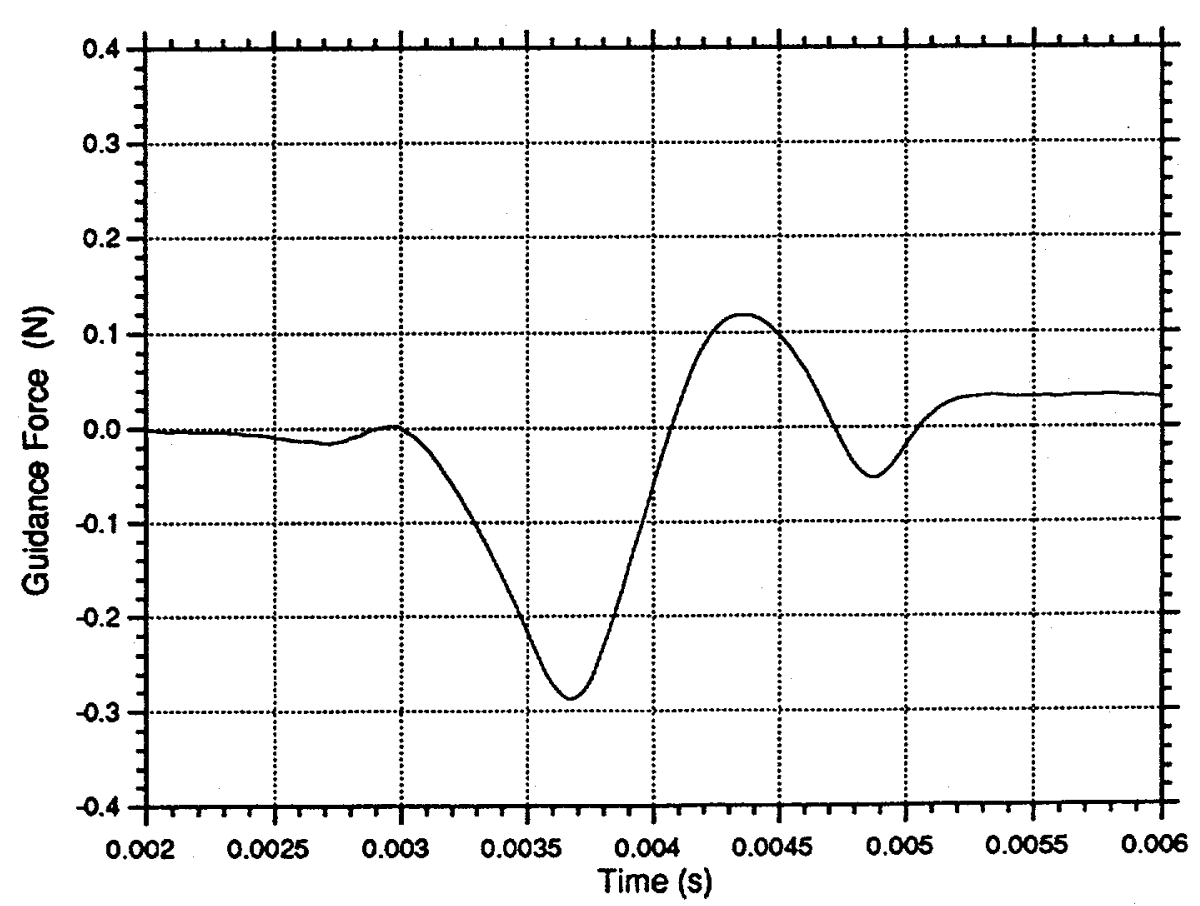

FIGURE 3.28b Experimental Guidance Force Impulse as a Function of Time for Two Five-Turn Parallel Coils $(26.4-\mathrm{mm} \times 62.0 \mathrm{~mm} \times$ 5.8-mm null-flux coil incident upon a $25.4-\mathrm{mm}$ square, 3.2-mm-thick, NdFeB permanent magnet at $y=10.3 \mathrm{~mm}$, $\mathrm{z}=6.67 \mathrm{~mm}$, and speed $=22.6 \mathrm{~m} / \mathrm{s}$ ) 


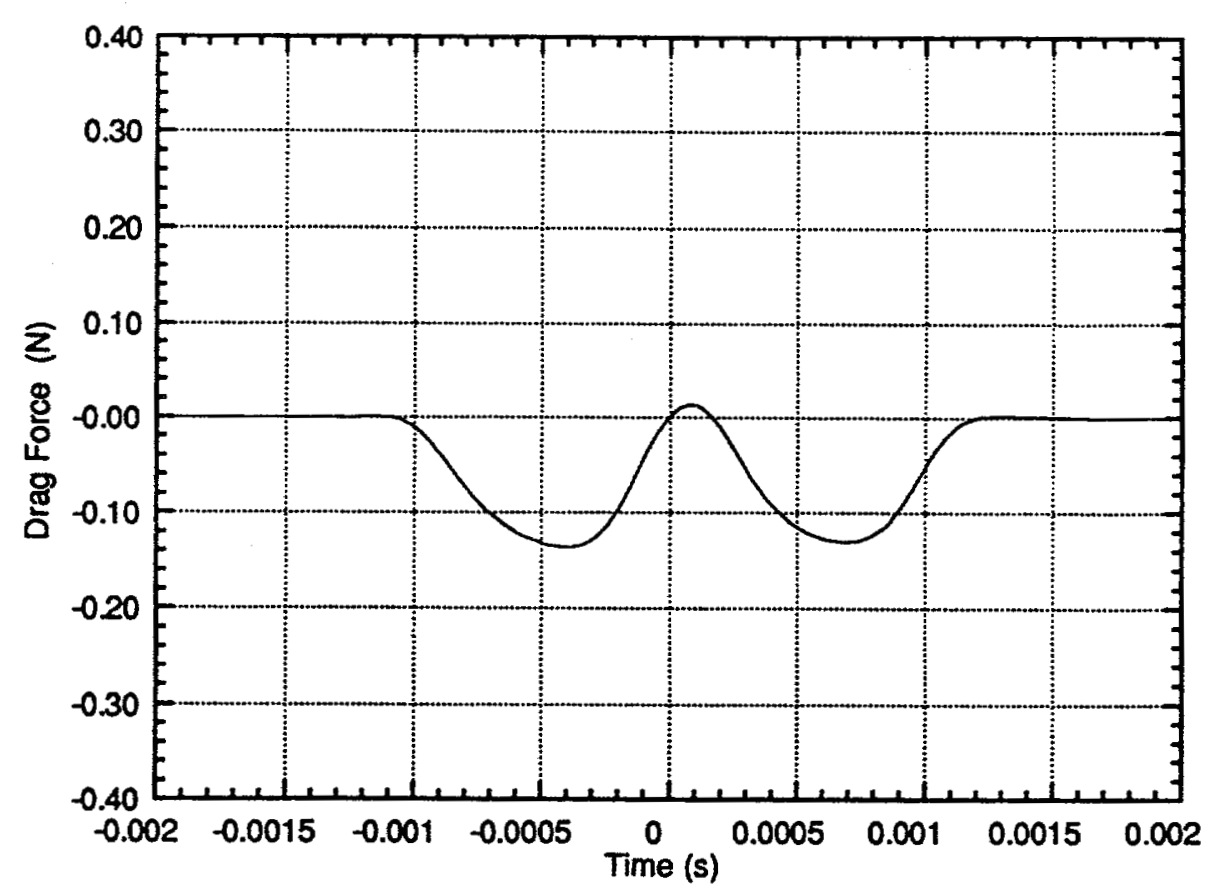

FIGURE 3.29a Computer-Calculated Drag Force Impulse as a

Function of Time for Two Five-Turn Parallel Coils (null-flux coil: length $=26.4-\mathrm{mm}$, height $=62.0 \mathrm{~mm}$, and thickness $=5.8 \mathrm{~mm}$; permanent magnet: length $=25.4 \mathrm{~mm}$, height $=25.4$, and thickness $=3.2 \mathrm{~mm}$; magnet coordinates: $y=-10.3 \mathrm{~mm}$, $z=6.67 \mathrm{~mm}$, and speed $=22.6 \mathrm{~m} / \mathrm{s}$ )

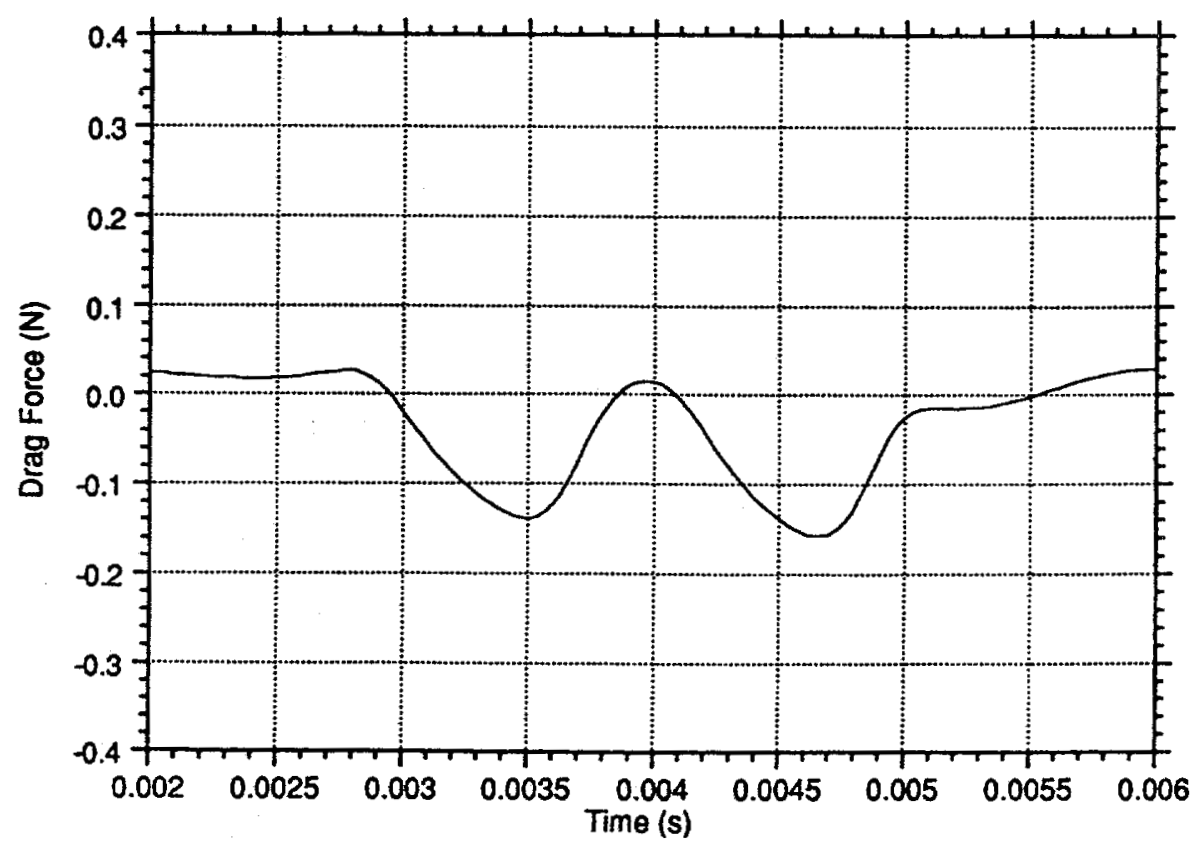

FIGURE 3.29b Experimental Drag Force Impulse as a Function of Time for Two Five-Turn Parallel Coils (null-flux coil: length = $26.4 \mathrm{~mm}$, height $=62.0 \mathrm{~mm}$, and thickness $=5.8 \mathrm{~mm}$; permanent magnet: length $=25.4 \mathrm{~mm}$, height $=25.4$, and thickness $=3.2 \mathrm{~mm}$; NdFeB permanent magnet at $\mathrm{y}=-10.3 \mathrm{~mm}$, $z=6.67 \mathrm{~mm}$, and speed $=22.6 \mathrm{~m} / \mathrm{s}$ ) 


\section{Conclusions}

The figure-eight-shaped null-flux coil suspension is an important maglev concept developed in Japan during the past decade. The concept has many advantages over other suspension systems. In particular, it provides very high lift-to-drag or lift-to-guidance ratios with a relatively simple configuration.

A computer code named "COILGDWY" was developed at ANL to simulate the performance of the figure-eight-shaped null-flux coil suspension system. The code can be used to simulate a superconducting coil (or vehicle coil) moving above a figure-eight-shaped null-flux coil guideway. Three-dimensional magnetic forces acting on the vehicle coils as functions of time or displacement can be determined. Design parameters for a maglev system can be obtained from the simulations.

A laboratory model consisting of a rotating PVC drum mounted with several groups of figure-eight-shaped null-flux coils interacting with a permanent magnet was constructed for the verification of the computer code. Transient magnetic forces were measured and compared with the results obtained from the computer model. Good agreement between the experimental results and the computer simulation was obtained. Although the verification was made at a small scale, the computer code is expected to be valid for large-scale simulations because of the linearity of the EDS maglev system. 


\section{References}

1. Power, J.R., and G.T. Danby, 1969, "Electromagnetic Inductive Suspension and Stabilization System for Ground Vehicle," United States Patent no. 3470828, Oct.

2. He, J.L., and D.M. Rote, 1993, "Double-Row Loop-Coil Configuration for EDS Maglev Suspension, Guidance, and Magnetic Guideway Switching," Institute of Electrical and Electronics Engineers, New York, Transactions on Magnetics, 29(6):2956-2958, Nov.

3. Fujiwara, S., and T. Fujimoto, 1989, "Characteristics of the Combined Levitation and Guidance System Using Ground Coils on the Side Wall of the Guideway," Proceedings of the International Conference on Maglev '89, July, pp. 241-244.

4. He, J.L., D.M. Rote, and H.T. Coffey, 1993, "Electromagnetic Forces of the CrossConnected Figure-Eight-Shaped Null-Flux Coil Suspension System," Proceedings of the International Conference on Maglev '93, May, pp. 64-70.

5. He, J.L., D.M. Rote, and H.T. Coffey, 1993, "Applications of the Dynamic Circuit Theory to Maglev Suspension," Institute of Electrical and Electronics Engineers, New York, Transactions on Magnetics, 29(6):4153-4164, Nov.

6. Grover, F.W., 1962, Inductance Calculations, Dover Publications, Inc., New York, N.Y.

7. Nasar, S.A., and I. Boldea, 1976, Linear Motion Electric Machines, Wiley-Interscience Publication, John Wiley and Sons, New York, N.Y. 